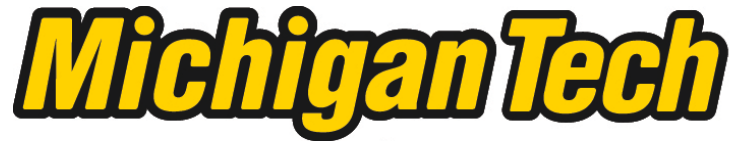 \\ Michigan Technological University Create the Future Digital Commons @ Michigan Tech
}

2015

\section{BLOOD TYPING DEVICE WITHOUT REAGENTS: SENSING ELECTRODES TO REPLACE OPTICS}

Nupur Bihari

Michigan Technological University

Follow this and additional works at: https://digitalcommons.mtu.edu/etds

Part of the Electrical and Computer Engineering Commons Copyright 2015 Nupur Bihari

\section{Recommended Citation}

Bihari, Nupur, "BLOOD TYPING DEVICE WITHOUT REAGENTS: SENSING ELECTRODES TO REPLACE OPTICS", Master's Thesis, Michigan Technological University, 2015.

https://doi.org/10.37099/mtu.dc.etds/1001

Follow this and additional works at: https://digitalcommons.mtu.edu/etds

Part of the Electrical and Computer Engineering Commons 
BLOOD TYPING DEVICE WITHOUT REAGENTS: SENSING ELECTRODES

TO REPLACE OPTICS

By

Nupur Bihari

\begin{abstract}
A THESIS
Submitted in partial fulfillment of the requirements for the degree of MASTER OF SCIENCE

In Electrical Engineering
\end{abstract}

MICHIGAN TECHNOLOGICAL UNIVERSITY

2015

(C) 2015 Nupur Bihari 

This thesis has been approved in partial fulfillment of the requirements for the Degree of MASTER OF SCIENCE in Electrical Engineering.

Department of Electrical \& Computer Engineering

\author{
Thesis Advisor: $\quad$ Dr. Paul L. Bergstrom \\ Committee Member: Dr. Adrienne R. Minerick \\ Committee Member: Dr. Glen E. Archer \\ Department Chair: Dr. Daniel R. Fuhrmann
}





\section{Contents}

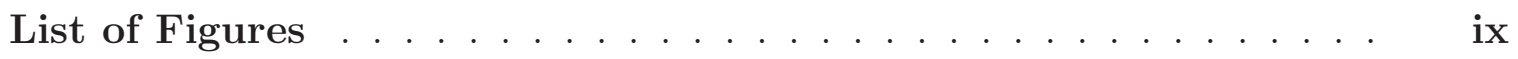

List of Tables . . . . . . . . . . . . . . . . . xvii

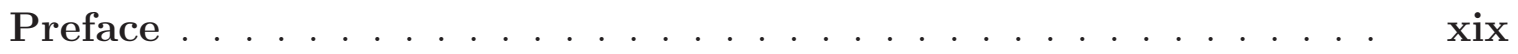

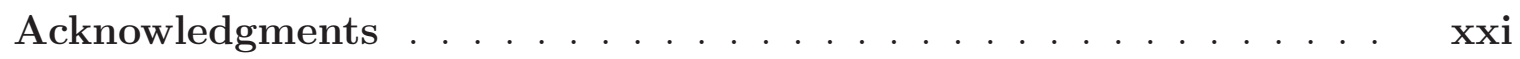

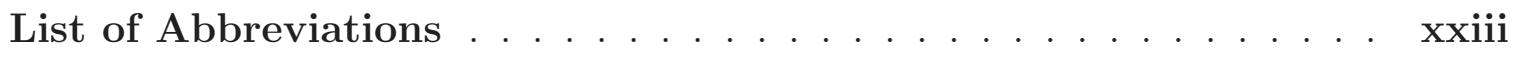

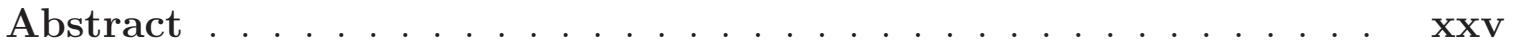

1 Introduction . . . . . . . . . . . . . . . . . . 1

1.1 Motivation . . . . . . . . . . . . . . . . . . . . . 1

1.2 Blood Types . . . . . . . . . . . . . . . . . . . . . . 3

1.2.1 ABO System of Blood Typing . . . . . . . . . . . . 3

1.2.2 Rh System of Blood Typing . . . . . . . . . . . . . 4

1.3 Pre-existing Methods of Blood Typing . . . . . . . . . . . 4

1.4 Dielectrophoresis . . . . . . . . . . . . . . 5

1.5 Objectives ............................. 6 
2 Theory and Design ....................... 9

2.1 Theory . . . . . . . . . . . . . . . . . . . . . . . . 9

2.1.1 Skin Depth Analytical Calculation . . . . . . . . . . 10

2.1.2 Lumped Element Analysis . . . . . . . . . . . . . . 12

2.1.3 COMSOL Model ... . . . . . . . . . . . . . . 14

2.2 Design . . . . . . . . . . . . . . . . . . . 17

2.2.1 Mask Designs for Glass slides . . . . . . . . . . . . . . . 18

2.2.2 Wafer Scale Design . . . . . . . . . . . . . . . . 22

2.2.3 Microfluidic Chamber . . . . . . . . . . . . . 45

2.2.4 Edge Connector SM3ZS067U310ABR120 . . . . . . . . 50

3 Fabrication Technology . . . . . . . . . . . . . . . 53

3.1 Early Process Development . . . . . . . . . . . . 53

3.2 Process Flow . . . . . . . . . . . . . . . . . 54

3.3 Choice of substrate . . . . . . . . . . . . . . . 57

3.3.1 Glass Slides . . . . . . . . . . . . . . . . . 57

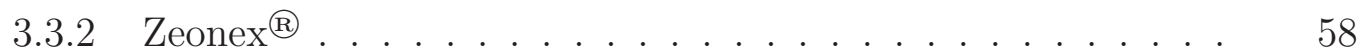

3.3.3 Borofloat $33{ }^{\circledR}$ Borosilicate Glass Wafers . . . . . . . . . . . 58

3.4 Sensing Electrodes . . . . . . . . . . . . . . . . . . 59

3.5 Metallization Layer for Electrodes . . . . . . . . . . . . . . . . 62

3.6 Passivation ........................... 64

3.7 Drive Electrodes . . . . . . . . . . . . . . . . . . 71 
3.8 Singulation . . . . . . . . . . . . . . . . . . . 72

3.9 Fluidic Chamber . . . . . . . . . . . . . . . . . 73

3.9.1 Well and Chamber ................. 74

3.9.2 PDMS Bonding . . . . . . . . . . . . . 75

4 Testing and Results . . . . . . . . . . . . . . . . . 77

4.1 Instrumentation . . . . . . . . . . . . . . . . . 78

4.2 Treatment of RBCs with Glutaraldehyde . . . . . . . . . . . . 79

4.3 Capacitive Testing . . . . . . . . . . . . . . . 79

5 Conclusions and Future Work . . . . . . . . . . . . . . . . . . 97

5.1 Conclusions .......................... 97

5.2 Future Work . . . . . . . . . . . . . . . . 100

References ....................... 103 



\section{List of Figures}

2.1 Lumped element model for circuit analysis . . . . . . . . . . . . .

2.2 3Linear device model . . . . . . . . . . . . . . . . .

$2.32 \mathrm{D}$ simulation of electric potential over drive electrodes for $100 \mathrm{kHz}$ done in collaboration with Jeana Collins . . . . . . . . . . . .

2.4 2D simulation of electric potential over drive electrodes for $1 \mathrm{MHz}$ done in collaboration with Jeana Collins . . . . . . . . . . . . . .

2.5 2D simulation of electric potential over drive electrodes and sensing electrodes for $100 \mathrm{kHz}$ with no passivation done in collaboration with Jeana Collins . . . . . . . . . . . . . . . . . . .

2.6 2D simulation of electric potential over drive electrodes and sensing electrodes for $1 \mathrm{MHz}$ with no passivation done in collaboration with Jeana Collins . . . . . . . . . . . . . . . . . . .

2.7 2D simulation of electric potential over drive electrodes and sensing electrodes for $100 \mathrm{kHz}$ with $25 \mathrm{~nm}$ passivation done in collaboration with Jeana Collins . . . . . . . . . . . . . . . . . . . . 
2.8 2D simulation of electric potential over drive electrodes and sensing electrodes for $1 \mathrm{MHz}$ with $25 \mathrm{~nm}$ passivation done in collaboration with Jeana Collins . . . . . . . . . . . . . . . . .

$2.92 \mathrm{D}$ simulation of electric potential over drive electrodes at $100 \mathrm{kHz}$ and sensing electrodes at $32 \mathrm{kHz}$ with $25 \mathrm{~nm}$ passivation done in collaboration with Jeana Collins . . . . . . . . . . . . . .

$2.102 \mathrm{D}$ simulation of electric potential over drive electrodes at $1 \mathrm{MHzand}$ sensing electrodes at $32 \mathrm{kHz}$ with $25 \mathrm{~nm}$ passivation done in collaboration with Jeana Collins . . . . . . . . . . . . .

2.11 Proof plot of sensing electrode mask . . . . . . . . . . . .

2.12 Proof plot of 3Linear sensing electrode . . . . . . . . . . . .

2.13 Proof plot of 7Linear sensing electrode . . . . . . . . . . . 24

2.14 Proof plot of 3Circular sensing electrode . . . . . . . . . . 25

2.15 Proof plot of 7Circular sensing electrode . . . . . . . . . 25

2.16 Proof plot of metallization layer . . . . . . . . . . . . 26

2.17 Proof plot of metallization die . . . . . . . . . . . . 27

2.18 Proof plot of metallization layer overlaid on sensing electrode layer . $\quad 27$

2.19 Device referencing scheme for wafer scale processing . . . . . . . 28

2.20 Layout of mask for sensing electrodes . . . . . . . . . . . . . 29

2.21 3Linear design of sensing electrodes . . . . . . . . . . . . . . . . 29

2.22 3Linear design of sensing electrodes showing truncated leads . . . . 30 
2.237 Linear design of sensing electrodes . . . . . . . . . . . .

2.24 3Circular design of sensing electrodes . . . . . . . . . . . . . .

2.25 Close up of 3 Circular electrode showing $3 \mu \mathrm{m}$ spacing of leads . . . .

2.267 Circular design of sensing electrodes . . . . . . . . . . . .

2.27 Close up of 7 Circular electrode showing $7 \mu \mathrm{m}$ spacing of leads . . . .

2.28 3Curved design of sensing electrodes . . . . . . . . . . . . .

2.297 Curved design of sensing electrodes . . . . . . . . . . . .

2.30 Alignment marks on sensing electrode mask . . . . . . . . . . .

2.31 Metallization layer mask . . . . . . . . . . . . . . . . . 36

2.32 Contact areas for aligning sensing layer to metallization layer . . . . 36

2.33 Close up view of metallization layer showing a die . . . . . . . . . 37

2.34 Alignment marks on metallization mask . . . . . . . . . . 38

2.35 Alignment of sensing electrodes to metallization layer in 3Linear de-

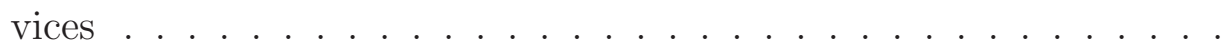

2.36 Alignment of sensing electrodes to metallization la0yer in 7Linear de-

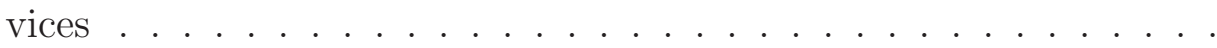

2.37 Alignment marks of metallization layer over sensing layer . . . . . .

2.38 Drive electrode mask . . . . . . . . . . . . . . . .

2.39 Close up of drive electrode layer showing a die . . . . . . . . . .

2.40 Quadruple drive electrodes . . . . . . . . . . . . . . . 42

2.41 Alignment marks on drive electrode layer . . . . . . . . . . . . . 
2.42 Alignment of drive layer to 3Linear sensing electrodes showing spacing and placement . . . . . . . . . . . . . . .

2.43 Alignment of drive layer to 3Linear sensing electrodes showing spacing

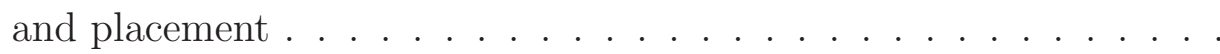

2.44 Alignment marks on sensing, metallization and drive layers . . . . .

2.45 Proof plot of fluidic chamber . . . . . . . . . . . . . .

2.46 Proof plot of fluidic chamber showing $1 \mathrm{~mm}$ chamber, inlet and outlet

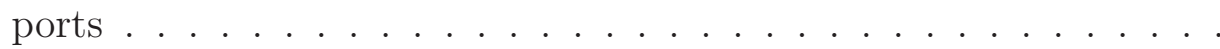

2.47 Design of microfluidic chamber mask . . . . . . . . . . . . . 47

2.48 Close up of chamber layer showing a die . . . . . . . . . . . . 48

2.49 Close up of chamber layer showing microfluidic chamber and inlet port

2.51 Close up of device masks . . . . . . . . . . . . . . . . . . 50

2.52 Eagle drawing of card edge connector SM3ZS067U310ABR120 . . . 51

3.1 Fabrication procedure . . . . . . . . . . . . 56

(a) Borofloat33 glass substrate . . . . . . . . . . . 56

(b) UV Photolithography ................. 56

(c) Patterns as seen after development . . . . . . . . . . . 56

(d) Sputter deposition of ITO . . . . . . . . . . . . 56

(e) Sample after ultrasonic liftoff .............. 56

(f) Alignment of metallization layer . . . . . . . . . . . . 56 
(g) Photoresist after development . . . . . . . . . . . 56

(h) $\mathrm{Cr} / \mathrm{Au}$ sputter deposition for metal leads and contacts . . . . 56

(i) Sample after second ultrasonic liftoff . . . . . . . . . . . 56

(j) Sample after passivation ................ 56

3.2 Optical transmission of Zeonex in visible and near-visible range [1] .

3.3 Parylene-C film showing defects . . . . . . . . . . . . . .

(a) Defects in Parylene-C layer $1 \ldots \ldots$. . . . . . . .

(b) Defects in Parylene-C layer $2 \ldots \ldots . \ldots$

(c) Defects in Parylene-C layer $3 \ldots \ldots$. . . . . . . .

3.4 Masked wafer for $\mathrm{HfO}_{2}$ deposition . . . . . . . . . . . . .

3.5 Comparison of LCR test data between Parylene coated and hafnia coated device. This data is for the same device B45 which was tested twice, with two different dielectrics. . . . . . . . . . . .

3.6 Final fabricated device sized to a $\$ 1$ coin for comparison . . . . . .

4.1 Day to day variability in measured values of capacitance in 3Circular device showing high capacitance on Day 2. This was attributed to the integrity of the Parylene layer being compromised after initial testing. This measurement was done in collaboration with Dr. Hector Moncada. . . . . . . . . . . . . . . . . . 
4.2 Day to day variability in measured values of capacitance in 7Linear device. This was attributed to the integrity of the Parylene layer being compromised after initial testing. This measurement was done in collaboration with Dr. Hector Moncada. . . . . . . . . . . .

4.3 Difference in capacitance with and without PS beads for 7Circular device showing inconsistent results, attributed to non-uniformity of passivation. This measurement was done in collaboration with Dr. Hector Moncada. . . . . . . . . . . . . . . . . . .

4.4 Difference in resistance with and without PS beads for 7Circular device showing a clear change in measured values with the analyte was changed. This measurement was done in collaboration with Dr. Hector

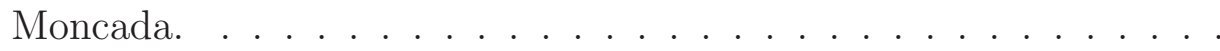

4.5 Difference in capacitance with and without PS beads for 3Circular device showing discernible difference between values obtained for different analytes. This measurement was done in collaboration with Dr. Hector Moncada. . . . . . . . . . . . . . . . . . .

4.6 Difference in resistance with and without PS beads for 3Circular device showing discernible difference between values obtained for different analytes. This measurement was done in collaboration with Dr. Hector Moncada. . . . . . . . . . . . . . . . . 
4.7 Difference in capacitance with and without PS beads for 7Linear device showing discernible difference between values obtained for different analytes. This measurement was done in collaboration with Dr. Hector

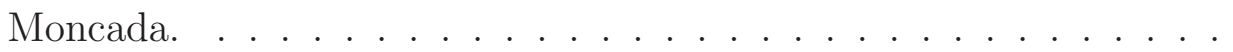

4.8 Difference in resistance with and without PS beads for 7Linear device showing inconsistent data. This was atrributed to a pure resistance measurement not taking into account all factor at play, leading to a need to gather data on phase angle and impedance. This measurement was done in collaboration with Dr. Hector Moncada. . . . . . . . .

4.9 Difference in capacitance with and without PS beads for 3Circular device showing discernible difference in values for different analytes. This measurement was done in collaboration with Dr. Hector Moncada. .

4.10 Difference in resistance with and without PS beads for 3Circular device showing inconsistent data. This was atrributed to a pure resistance measurement not taking into account all factor at play, leading to a need to gather data on phase angle and impedance. This measurement was done in collaboration with Dr. Hector Moncada. . . . . . . . .

4.11 Difference in capacitance with and without PS beads for 3Linear device showing discernible difference in values for different analytes. This measurement was done in collaboration with Dr. Hector Moncada. . 
4.12 Difference in resistance with and without PS beads for 3Linear device showing inconsistent data attributed to bad contact behavior. This measurement was done in collaboration with Dr. Hector Moncada. .

4.13 Comparison between different probe needles $7 \mathrm{x}$ and $7 \mathrm{x}$ short while performing the same measurement showing $7 \mathrm{x}$ needles yielding higher values of capacitance. This measurement was done in collaboration with Dr. Hector Moncada. . . . . . . . . . . . . . . . . . . . . 94

4.14 Differential measurement of PBS and RBCs (1:65 concentration) showing higher sensitivity at $1 k H z, 1 \mathrm{~V}$ for a solution conductivity of $0.05 \mathrm{~S} / \mathrm{m}$. This measurement was done in collaboration with Dr. Hector

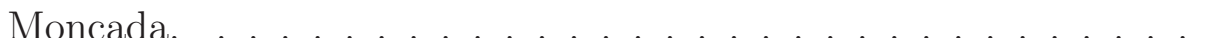

4.15 Differential measurement of PBS and RBCs (1:65 concentration) showing highest sensitivity at $32 \mathrm{kHz}, 1 \mathrm{~V}$ for a solution conductivity of $0.01 \mathrm{~S} / \mathrm{m}-$ the ideal case for alpha prototype. This measurement was done in collaboration with Dr. Hector Moncada. . . . . . . . . . . . 96 


\section{List of Tables}

3.1 Process flow for sensing electrodes using dark field masks and positive

photoresist ............................. 60

3.2 Process flow for sensing electrodes using clear field masks and negative photoresist ........................... 60

3.3 Sheet resistance for 80nm sputter deposited ITO film without annealing and with a hotplate anneal at $200^{\circ} \mathrm{C}$ for 30 mins . . . . . . . . 62

3.4 Process flow for metallization layer using clear field masks and negative

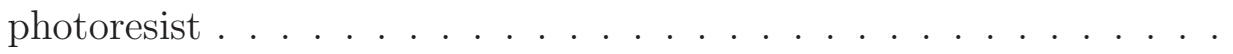

3.5 LCR test data of B45 showing inconsistencies due to passivation . .

3.6 LCR test data for hafnia coated device B45 after Parylene-C was

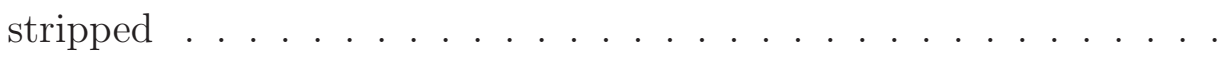

3.7 Process flow for drive electrodes using clear field masks and negative

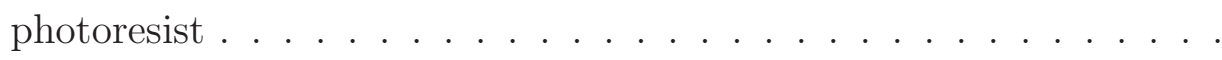

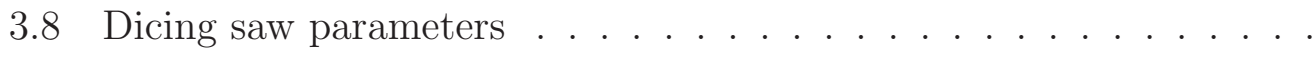

3.9 Process flow for fluidic chamber using SU8 2025 resist . . . . . . . . 
4.1 Mean and Standard Deviation Capacitance and Resistance values of

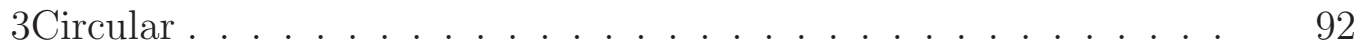

4.2 Mean and Standard Deviation Capacitance and Resistance values of 3Linear . . . . . . . . . . . . . . . . . . . . . . . . . . . .

4.3 Impedance data for 3Linear device (standard deviation shown in ital$i c s) \ldots \ldots \ldots \ldots \ldots \ldots$

4.4 Phase Angle data for 3Linear device (standard deviation shown in ital-

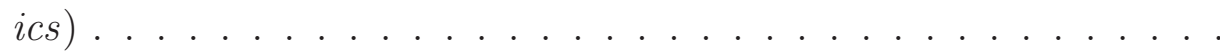




\section{Preface}

This thesis is based on my Master's research work conducted at Michigan Technological University under the guidance of Dr. Paul Bergstrom, during the period May 2014 to August 2015. The COMSOL modeling was completed by Jeana Collins in collaboration with me, with inputs from Dr. Hector Moncada and Zhichao Wang. The process development and device fabrication effort was completed by me with the assistance of Zhichao Wang. Dr. Hector Moncada and Jeana Collins also contributed. The testing of these devices was performed by Dr. Hector Moncada and myself, with assistance from Zhichao Wang. The research team intends to submit this work for publication in the near future. 



\section{Acknowledgments}

Foremost, I would like to express my sincerest gratitude to my advisor, Dr. Paul Bergstrom who believed in me and supported me throughout my time at Michigan Tech. He provided me with a great opportunity and was a constant source of guidance and direction. I am honored and immensely privileged to be his student.

I thank Dr. Adrienne Minerick who guided me throughout and provided very valuable feedback. She was full of ideas and without her this thesis would not have been possible.

I thank Dr. Bergstrom, Dr. Minerick and Dr. Archer for being on my committee and providing feedback, guidance and words of encouragement.

Mark Sloat, Mike Chase, Chuck Sannes and Dr. Chito Kendrick, your contribution in supporting the facility will always be appreciated.

I am indebted to my research team, Zhichao Wang, Jeana Collins and Dr. Hector Moncada Hernandez for taking the time out from teaching and research to help on this project. You are not only the best team I could have hoped for, but also friends I will cherish for life. The author acknowledges the support of Jeana Collins with the theory and modeling, especially the COMSOL model. Zhichao Wang, Jeana Collins 
and Dr. Hector Moncada were instrumental in the process of fabricating the device. The author acknowledges the support of Hector Moncada, who was responsible for the majority of the testing effort. Zhichao Wang and Jeana Collins were also actively involved in testing devices.

I acknowledge Dr. Thomas Daunais and Sterling Prince for teaching me almost everything I know in the Microfabrication Facility and being the two people I know I can always depend on.

My family has been an unwavering source of support. When the going got tough they would offer words of encouragement and tell me that they love me unconditionally.

Richard Buis and everyone at the Canterbury House has been my family here, and thanks to them Houghton feels like home. I am indebted to my many friends for their warmth and generosity. Kishan Bellur's contributions to the many hours spent debugging LaTeX code are highly appreciated.

Lastly, I thank Michigan Technological University, National Science Foundation, Dr. Bergstrom and the Microfabrication Facility for financial support. 


\section{List of Abbreviations}

\begin{tabular}{|c|c|}
\hline ITO & Indium Tin Oxide \\
\hline TMAH & Tetramethylammonium Hydroxide \\
\hline PVD & Physical Vapor Deposition \\
\hline CVD & Chemical Vapor Deposition \\
\hline VPD & Vapor Phase Deposition \\
\hline $\mathrm{RBC}$ & Red Blood Cells \\
\hline LCR & Inductance Capacitance Resistance \\
\hline PDMS & Polydimethylsiloxane \\
\hline SPA & Semiconductor Parameter Analyzer \\
\hline DEP & Dielectrophoresis \\
\hline $\mathrm{nDEP}$ & Negative Dielectrophoresis \\
\hline $\mathrm{pDEP}$ & Positive Dielectrophoresis \\
\hline $\mathrm{Pa}-\mathrm{C}$ & Parylene-C \\
\hline $\mathrm{CD}$ & Critical Dimension \\
\hline PBS & Phosphate Buffered Saline \\
\hline $\mathrm{PS}$ & Polystyrene Beads \\
\hline cof & Crossover Frequency \\
\hline
\end{tabular}





\section{Abstract}

There is need to develop a fast and efficient procedure for detecting blood type that makes use of an electronic measurement to minimize human error and is portable so that it can be used in triage situations. A process to develop a crossover frequency based blood typing device is described. Alternating field drives red blood cells over the sensing electrodes and depending on the capacitance measured between pairs of sensing electrodes, the position of these RBCs can be determined. The convergence/divergence of these cells at a particular frequency determines blood type, since each type has a characteristic frequency. This project aims to obviate the need for optics in blood typing by making use of a purely electric measurement.

A microdevice was fabricated with different designs of sensing electrodes. These electrodes were passivated using Parylene-C and hafnia. A fluidic chamber was placed over them, with inlet/outlet ports for RBCs suspended in solution. An LCR meter was used to measure capacitance between different pairs of electrodes. While testing is still in progress, preliminary data suggests measurable difference in capacitance between readings taken when the fluidic chamber was filled with just ultrapure water, phosphate buffered solution and solution containing RBCs, proving this method as a viable route to blood typing. 
Analytical calculations and COMSOL 4.4 simulations suggest coupling of sensing frequency to the driving frequency will necessitate switching in order to improve accuracy. Areas of high cell concentration were studied and these will be used for optimizing design of sensing electrodes to improve accuracy. 


\section{Chapter 1}

\section{Introduction}

\subsection{Motivation}

There exists a need to sense particles in an accurate and cost effective manner in the biological, chemical and environmental domains. Mortality rate is still high due to untimely and insufficient medical care. Disease detection and treatment is heavily dependent on biological reagents and trained personnel. Nolte et al. [2] describe amenable mortality as avoidable death. This is related to all cases where the role of the practitioner and medical care was non-optimum.

Disease detection is a field that relies heavily on optical analysis of biological samples, especially blood and tissue. This concept of amenable mortality is especially 
visible in triage situations, where timely medical care is of utmost importance. There exists a need to rapidly sense a difference between diseased cells in tissues (including blood) and healthy cells. Many diseases, such as cancer, have markers that exhibit themselves in the cell membrane. Cancer has been detected by measuring a change in the properties of diseased cells and comparing the results to those obtained from healthy cells [3]. Similarly malaria afflicted cells have been isolated [4]. These have been done by exposing the samples to an electric field and observing their behavior.

Triage situations often necessitate rapid blood transfusions and in most cases, to date, universal donor blood type O- is used. This has resulted in a shortage of blood actually required for patients with blood type O-. Thus, there is a need to have a portable method of sensing blood type of such critically injured patients in order to provide optimum medical care without diminishing blood supplies that are required for other patients.

Rapid blood sensing technology, as detailed in this document, aims to achieve this objective. A portable, handheld device is described which has the capability to tell blood type based on an electrical measurement. 


\subsection{Blood Types}

The International Society of Blood Transfusion (ISBT) recognizes 33 different systems of blood typing based on different biological markers [5]. Out of these, two systems are most commonly used: the $\mathrm{ABO}$ and the Rh system.

\subsubsection{ABO System of Blood Typing}

The ABO system of blood typing was discovered by Karl Landsteiner in 1900 [6]. It differentiates between blood types based on the presence or absence of antigens on the surface of the red blood cells. These antigens are primarily oligosaccharides, i.e., sugar polymers that determine a persons immunological tolerance. Two antigens, A and $\mathrm{B}$ have been recognized. A person may have either one of those, or both ( $\mathrm{AB}$ type) or neither (O type).

The opposite antibodies are present in blood plasma. While these antibodies are useful in fighting infection as they tend to agglomerate and break apart foreign blood cells, this becomes a problem in case of blood transfusion. 


\subsubsection{Rh System of Blood Typing}

The Rh system was discovered by Karl Landsteiner and Alexander S. Wiener [7] in 1940 after a case of a mothers blood getting agglutinized by the fetal blood. It was observed that there was a difference in an antigen contained by the mothers blood and her fetus. This antigen was further studied and was named the Rh factor. The presence or absence of this antigen is sensed in order to determine blood type.

\subsection{Pre-existing Methods of Blood Typing}

The most commonly used method of blood typing till date requires biological reagents. A small sample of blood is extracted and is made to react with different known antibodies. The reaction of the antibodies with the antigens provides information about blood type. This method requires biological reagents and trained laboratory personnel. Also, one measurement takes several minutes in case of immediate-spin cross-matching [8], the most rudimentary procedure which does not include complete ABO-Rh system typing [9]. A more accurate method, called Electronic crossmatching is a computer assisted way of testing compatibility between blood types from a donor to a recipient. This method too relies heavily on trained technicians and takes up to an hour to complete. The results may not be entirely objective since the scope for 
human error is non-trivial.

Both of these methods are time and labor intensive and for accurate results must follow a number of guidelines set by Clinical Pathology Accreditation [10], an association of trained pathologists and hematologists. The large number of limitations of these preexisting methods of blood typing [11] necessitate a new system that alleviates the need for trained staff and is less time intensive.

\subsection{Dielectrophoresis}

The term dielectrophoresis was first defined by Herbert A. Pohl for particles experiencing a polarization force in a non-uniform electric field [12]. The very same effect was then seen in yeast cells [13] and was tested by altering the field gradient and intensity. It was observed that the yeast cells would accumulate at the area of highest field intensity, in this case, the tip of the driving electrode.

Dielectrophoresis has been used as a method of identification and separation of many different particles, the most notable being cancer cells from blood based on difference in plasma membrane capacitance [3]. The same concept has been used to sense droplets in a capacitive sensor [14].

This eventually led to a study concerning the dielectrophoretic response of human 
blood cells. Human RBCs are polarizable, which implies that they show a dielectrophoretic response in a varying electric field. This effect was studied by Minerick et al. [15] and it was observed that RBCs tend to either be attracted or repelled by electrodes depending on the frequency. It was noticed during a frequency sweep that the cells are attracted to the driving electrode during a particular frequency band, and this band differs slightly between different blood types. The same effect is seen in case of repulsion too. Thus, there are two crossover frequencies, i.e., the frequencies at which attraction/repulsion behavior of the RBCs changes.

It was theorized that it is possible to characterize the RBCs to determine the two frequencies at which this behavior happens. This was previously done using optical means. RBCs were subjected to a frequency sweep in an alternating field and their response was optically measured and data on crossover frequencies was collected. There was a need to obviate the optical measurement system in lieu of a fully automated network of sensors to determine the behavior of cells at this crossover frequency.

\subsection{Objectives}

Objective One of this thesis was to design and interface parallel capacitive sensing electrodes with microfluidic chamber to sense cell location. This included: 
- Design and fabrication of sensing electrodes exploring linear and circular geometries using $\mathrm{Cr} / \mathrm{Au}$

- Optimization of experimental conditions such as frequency, voltage, conductivity etc.

- Design and analysis of COMSOL model to determine effect of passivation and for a visualization of the electric field in the sensing region

- Testing with Phosphate Buffer Solution (PBS) and Polystyrene (PS) beads to demonstrate difference between dry measurements, measurements with PBS and PBS $+\mathrm{PS}$ beads

- Testing with glutaraldehyde treated RBCs to show real world application of technology

- Fabrication of transparent sensing electrodes using Indium Tin Oxide (ITO) to enable corroboration of the electrical and optical measurements in the design

Objective two was to test ITO sensing electrodes concurrent with dielectrophoretic (DEP) manipulation of RBCs to ascertain response times and interference. This included:

- Design of electrodes and contact pads compatible with an edge connector 
- Patterning of drive electrodes over existing sensing platform for concurrent DEP manipulation of cells

- Preparing a set of devices that could be used for DC vs AC coupling analysis to determine maximum sensitivity to cell localization

The following chapters describe these objectives in more detail. Chapter 2 details the theory and modeling aspect of this thesis, focusing on a lumped element analysis and extracting information from it to create a COMSOL model depicting electric field gradients. Chapter 3 details the fabrication of the device, going into design constraints and challenges faced during this process. Chapter 4 details testing conditions and the results obtained. Chapter 5 describes all conclusions made in the preceding chapters and the scope for future work. 


\section{Chapter 2}

\section{Theory and Design}

\section{$2.1 \quad$ Theory}

The work described in this chapter was done to fulfill a part of objective one of this thesis, to build and simulate a model to mimic the function of the device. This was done in order to corroborate the experimental findings and also to predict the range of expected measurements. This in turn was required to select on-chip electronics for the alpha prototype. 


\subsubsection{Skin Depth Analytical Calculation}

The first step in the process was an analytical calculation that would help determine the approximate value of capacitance in the circuit for dry, wet and cell measurements.

Here, a dry measurement relates to an empty chamber. Hence, the relative dielectric constant of the medium is $\epsilon_{r}=1$.

A wet measurement relates to a chamber filled completely with PBS. Hence, $\epsilon_{r}=80$.

The height of the chamber was $70 \mu \mathrm{m}$. Hence, it was important to determine the extent of the field strength since all media were considered lossy dielectrics. An alternating field was used for both sensing and driving. The sensing electrodes were to be operated at a constant frequency of $32 \mathrm{kHz}$ on the alpha prototype. Hence, this frequency was chosen for skin depth calculations.

Complex permittivity was used to calculate the wave number and the skin depth as

shown in Equations 2.1 and 2.2 [16]. This was done in order to take into account the conductivity of the solution and variation of relative dielectric constant with frequency. 


$$
\begin{gathered}
\hat{\epsilon}=\epsilon_{r} \epsilon_{0}-j \frac{\sigma}{\omega} \\
\delta=\frac{1}{\kappa}=\frac{1}{\omega \sqrt{\mu \hat{\epsilon}}}
\end{gathered}
$$

where,

$\epsilon_{r}$ is relative permittivity

$\epsilon_{0}$ is permittivity of free space

$\sigma$ is conductivity of solution

$\omega$ is the angular frequency; $2 \pi f$

$\kappa$ is the wave number

$\delta$ is the skin depth.

The value of skin depth was calculated to be $6.41 \mathrm{~m}$. Since the skin depth was much higher than the height of the fluidic chamber it was concluded that the electric field would have an impact on the DEP response of the entire lossy medium.

Thus a way to calculate the values of these capacitances was sought and a lumped 
element model was recommended.

\subsubsection{Lumped Element Analysis}

A lumped element model was built to extract an approximate value of expected capacitances for a passivated device.

This was based on the circuit as shown in Figure 2.1

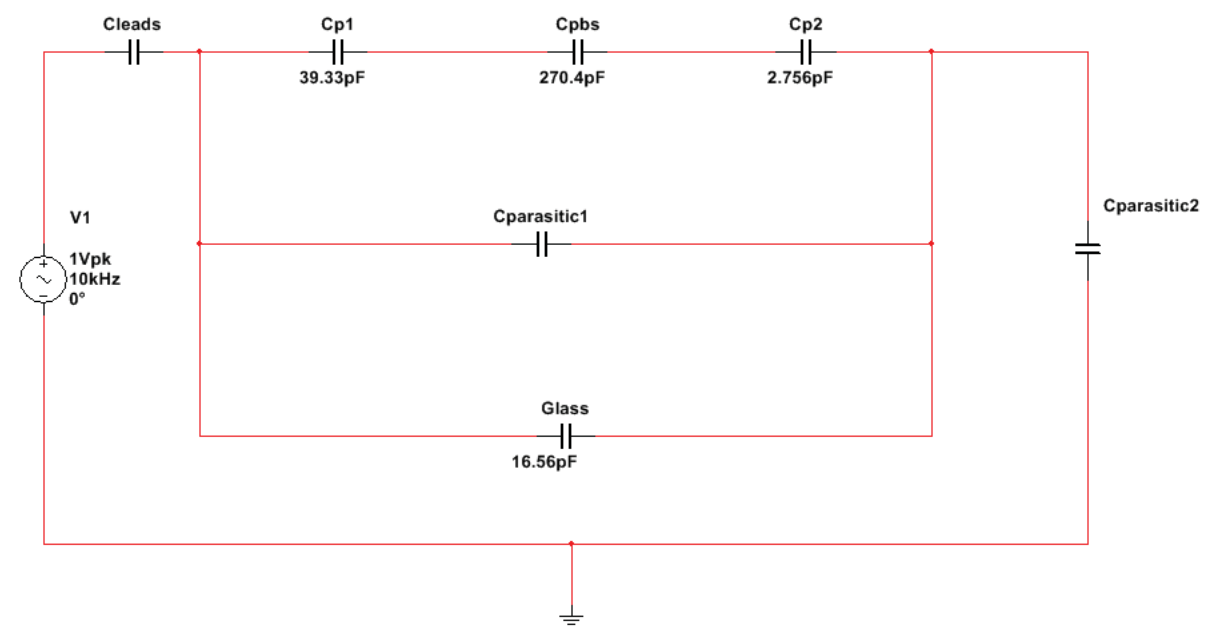

Figure 2.1: Lumped element model for circuit analysis

Yang et al. [17] describe a model for calculating capacitance based on geometries.

The equation for capacitance based on geometry is given by equation 2.3 [18].

$$
C=\frac{\epsilon \omega}{h}\left[1+\frac{2 h}{\pi \omega} \ln \left(\frac{\pi \omega}{h}\right)+\frac{2 h}{\pi \omega} \ln \left(1+\frac{2 t}{h}+2 \sqrt{\frac{t}{h}+\frac{t^{2}}{h^{2}}}\right)\right]
$$


This model takes into account the fringing fields as functions of width, height and thickness of features [18][19]. A calculation using this equation was done to find expected values of capacitance for 3Linear devices. The substrate was Borofloat33 glass obtained from University Wafers. Here $\epsilon_{r}$ was 4.9. $\epsilon_{r}$ for PBS was assumed to be 80. Parylene-C was chosen as the dielectric for modeling. This model was applied as shown in Figure 2.2.

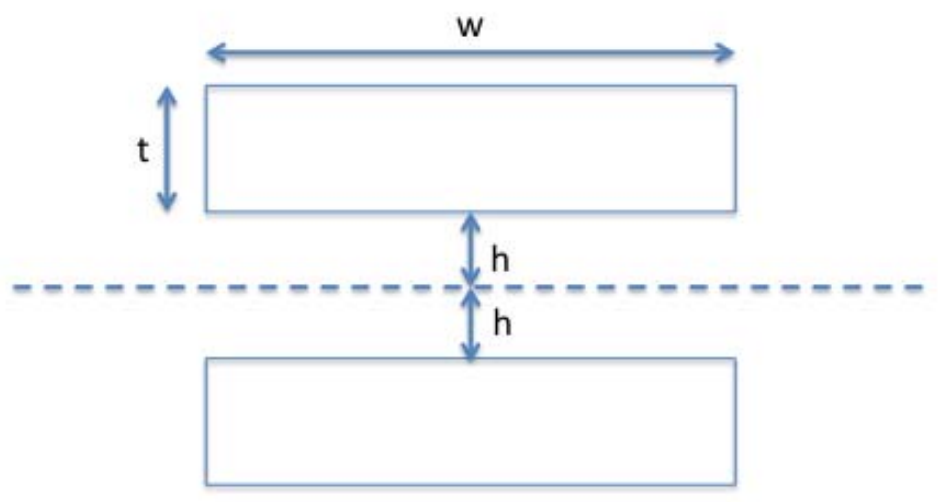

Figure 2.2: 3Linear device model

The values obtained for the given geometries were:

$$
\begin{aligned}
& C_{P 1}=3.933 \times 10^{-11} \mathrm{~F} \\
& C_{P 2}=2.786 \times 10^{-12} \mathrm{~F} \\
& C_{P B S}=2.704 \times 10^{-10} \mathrm{~F} \\
& C_{\text {glass }}=1.656 \times 10^{-11} \mathrm{~F}
\end{aligned}
$$

The equivalent capacitance was calculated as Equation 2.4. 


$$
C=C_{\text {glass }}+C_{P 2}+\left(\frac{1}{C_{P 1}}+\frac{1}{C_{P B S}}+\frac{1}{C_{P 2}}\right)^{-1}
$$

The expected value for a wet measurement of capacitance was calculated to be 3.769 $\mathrm{x} 10^{-11} \mathrm{~F}$. This value was much closer to the experimentally measured values as compared to other calculated methods which ignored the effects of fringing fields.

\subsubsection{COMSOL Model}

To graphically illustrate the distribution of the electric field lines various simulations using COMSOL 4.4 were done.

For ease of meshing and calculation, 2D simulations were preferred over 3D. There was a need to be able to visualize the field over the area which would experience the DEP force.

The 2D simulation taking into account only the drive electrodes is shown in Figure 2.3

Since the lower and upper limits for driving field were $100 \mathrm{kHz}$ and $1 \mathrm{MHz}$ respectively, simulations were completed for both frequencies. The simulation for $1 \mathrm{MHz}$ is shown in Figure 2.4. 
- $0.1 \mathrm{MHz}$

- $5 \mathrm{Vpp}(-2.5$ to 2.5$)$

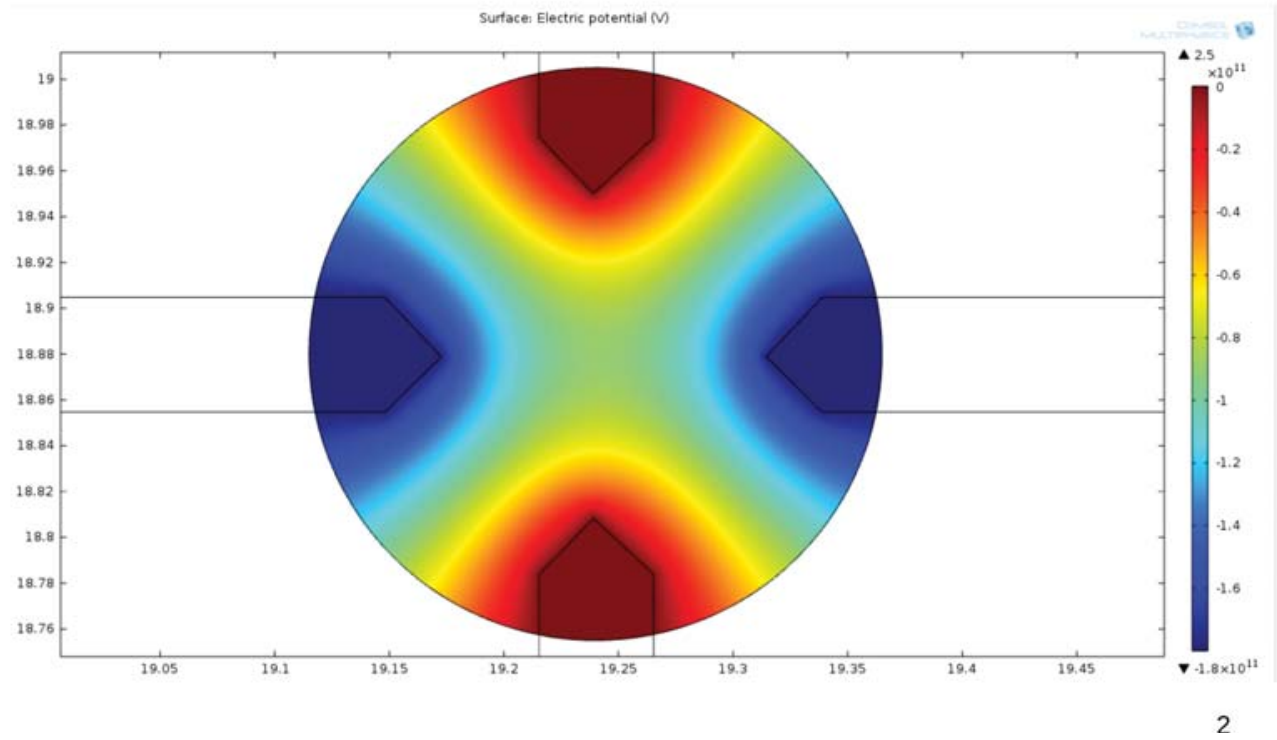

Figure 2.3: 2D simulation of electric potential over drive electrodes for $100 \mathrm{kHz}$ done in collaboration with Jeana Collins

It was seen that the electric field intensity was highest near the tip of the electrodes, which was expected and necessary since during pDEP the cells would be attracted to the quadruple array and downwards towards the substrate.

Next, the 3Linear drawing was added to the design to look at the effect sensing electrodes would have on this preexisting field. For these simulations the effect of passivation was ignored to get a baseline value which would be used to evaluate the effect of the dielectric film. Again, this was done for both the lower and upper frequency limits. This is shown in Figures 2.5 and 2.6.

Next, there was a need to evaluate the effect of passivation. A thin $25 \mathrm{~nm}$ hafnia film was chosen as the dielectric of choice. This had an impact on the field density as 
- $1.0 \mathrm{MHz}$

- $5 \mathrm{Vpp}(-2.5$ to 2.5$)$

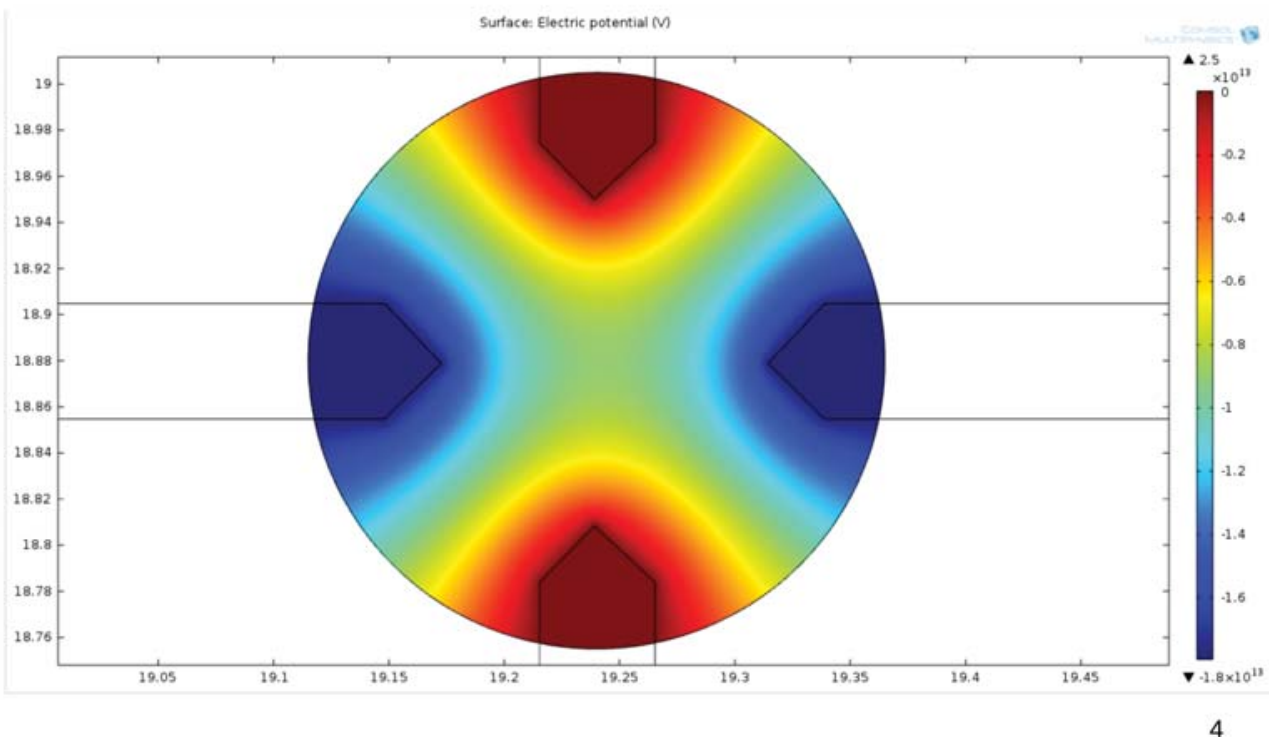

Figure 2.4: 2D simulation of electric potential over drive electrodes for $1 \mathrm{MHz}$ done in collaboration with Jeana Collins

shown in Figures 2.7 and 2.8 .

While the previous simulation looked at floating sensing electrodes, simulations were required to look at the effect of ac field generated by the sensing electrodes over the passivation. The sensing electrodes were given a frequency of $32 k H z$ which was consistent with the frequency of the chip on the alpha prototype. This is shown in Figures 2.9 and 2.10 .

It was observed that the sensing electrodes contributed to the measurement by generating a high intensity field around them. This would affect the DEP response of the cells. This simulation was helpful in drawing two conclusions: 
- $0.1 \mathrm{MHz}$

- $5 \mathrm{Vpp}(-2.5$ to 2.5$)$

- No passivation

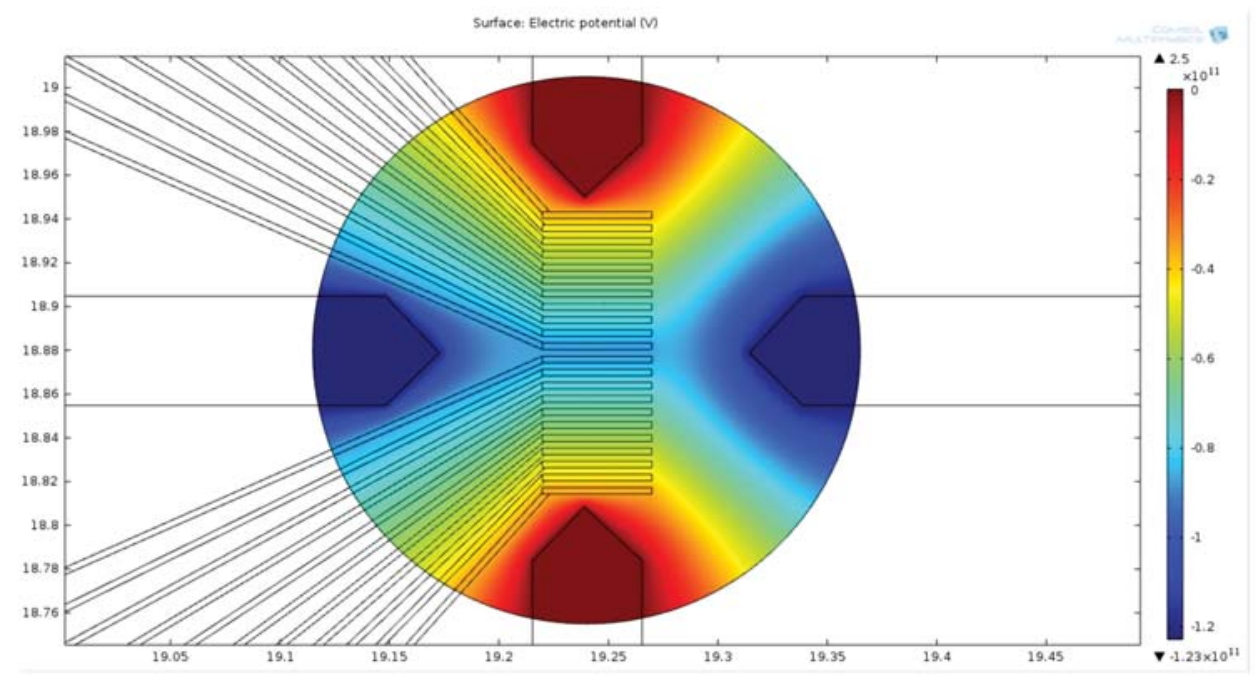

5

Figure 2.5: 2D simulation of electric potential over drive electrodes and sensing electrodes for $100 \mathrm{kHz}$ with no passivation done in collaboration with Jeana Collins

- There would be a need to alternate between switching the drive and the sensing electrodes on and off for the measurements to not be affected by the field generated by sensing electrodes.

- For future design and processing pixels could be placed in the areas with highest field intensity for more accurate and stronger response.

\subsection{Design}

During the first phase fabrication trials, all masks were designed for glass slides. After a round of preliminary testing, this design was elevated to wafer scale. Both these 
- $1.0 \mathrm{MHz}$

- $5 \mathrm{Vpp}(-2.5$ to 2.5$)$

- No passivation

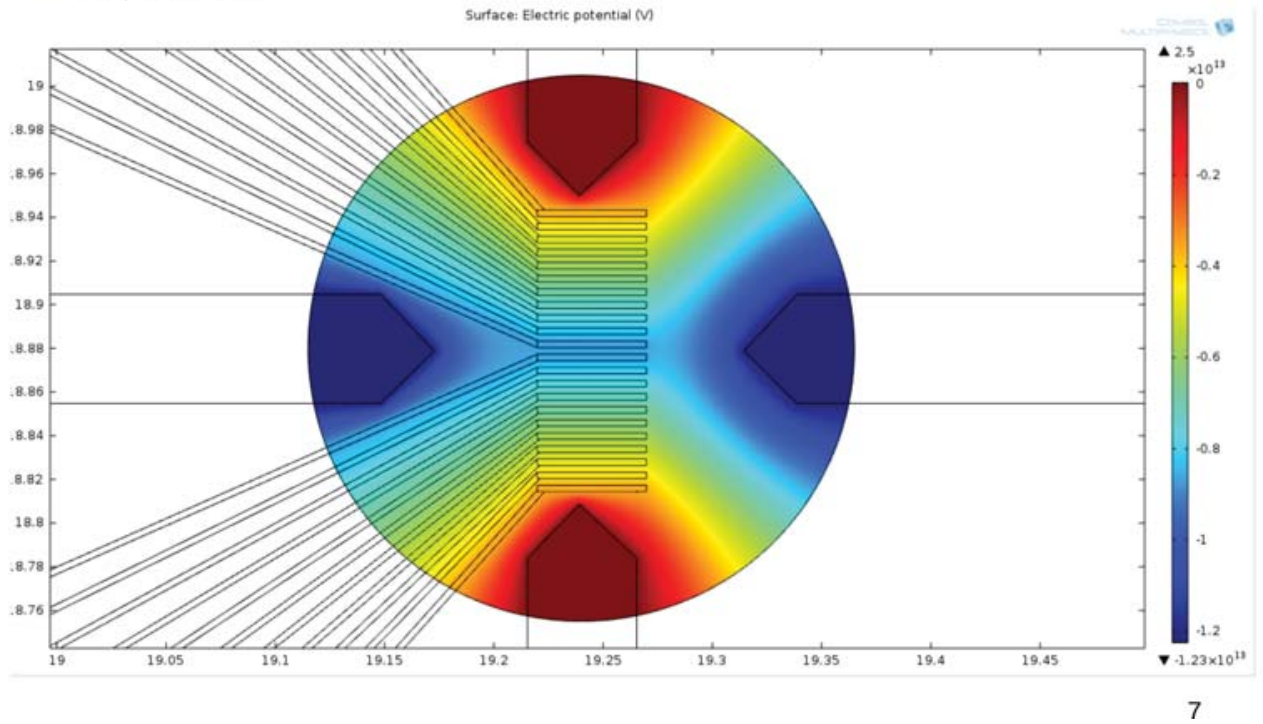

Figure 2.6: $2 \mathrm{D}$ simulation of electric potential over drive electrodes and sensing electrodes for $1 \mathrm{MHz}$ with no passivation done in collaboration with Jeana Collins

design processes are described below.

\subsubsection{Mask Designs for Glass slides}

A decision to design dark field masks for slide scale processing was made in order to utilize positive resist, which yields better liftoff features.

The mask for the sensing electrode layer is shown in Figure 2.11.

A set of parallel sensing electrodes was designed to sense the position of red blood cells. The distance between each of these sensing electrodes was decided to be $3 \mu \mathrm{m}$ 
- $0.1 \mathrm{MHz}$

- $5 \mathrm{Vpp}(-2.5$ to 2.5$)$

- Thin, low permittivity gap, $25 \mathrm{~nm}$

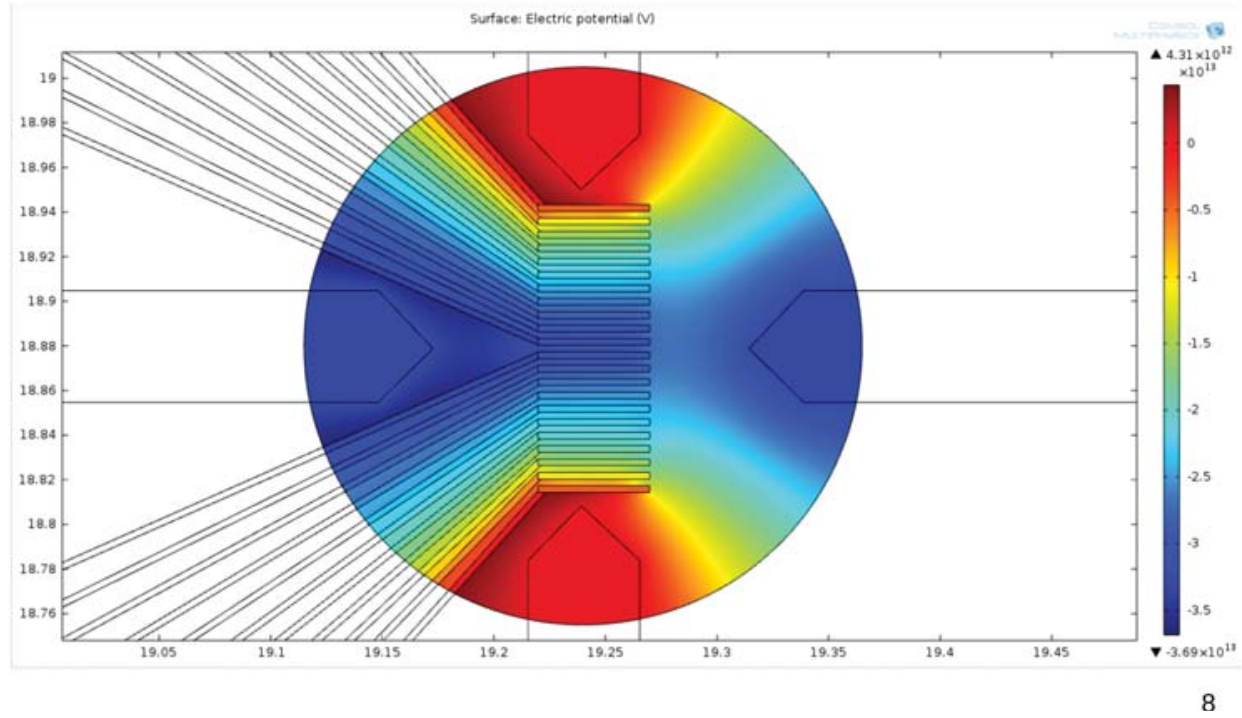

Figure 2.7: 2D simulation of electric potential over drive electrodes and sensing electrodes for $100 \mathrm{kHz}$ with $25 \mathrm{~nm}$ passivation done in collaboration with Jeana Collins

(Figure 2.12) and $7 \mu \mathrm{m}$ (Figure 2.13) in a linear array of equal lines and spaces [20]. The motivation behind choosing these dimensions was the fact that red blood cells vary between $2 \mu \mathrm{m}$ and $6 \mu \mathrm{m}$ in size [21]. Thus, a design that was near the lower limit of that range and another one close to the upper limit was desired.

Previous research [22] done by Leonard et al. demonstrated that at crossover frequency, red blood cells move in a concentric circular pattern, either away or close to the center of the quadruple drive electrodes. In order to better sense this response a circular array of electrodes was desired as an alternate design.

Concentric circles were designed, with the distance between elements $3 \mu \mathrm{m}$ and $7 \mu \mathrm{m}$. 
- $1.0 \mathrm{MHz}$

- $5 \mathrm{Vpp}(-2.5$ to 2.5$)$

- Thin, low permittivity gap, $25 \mathrm{~nm}$

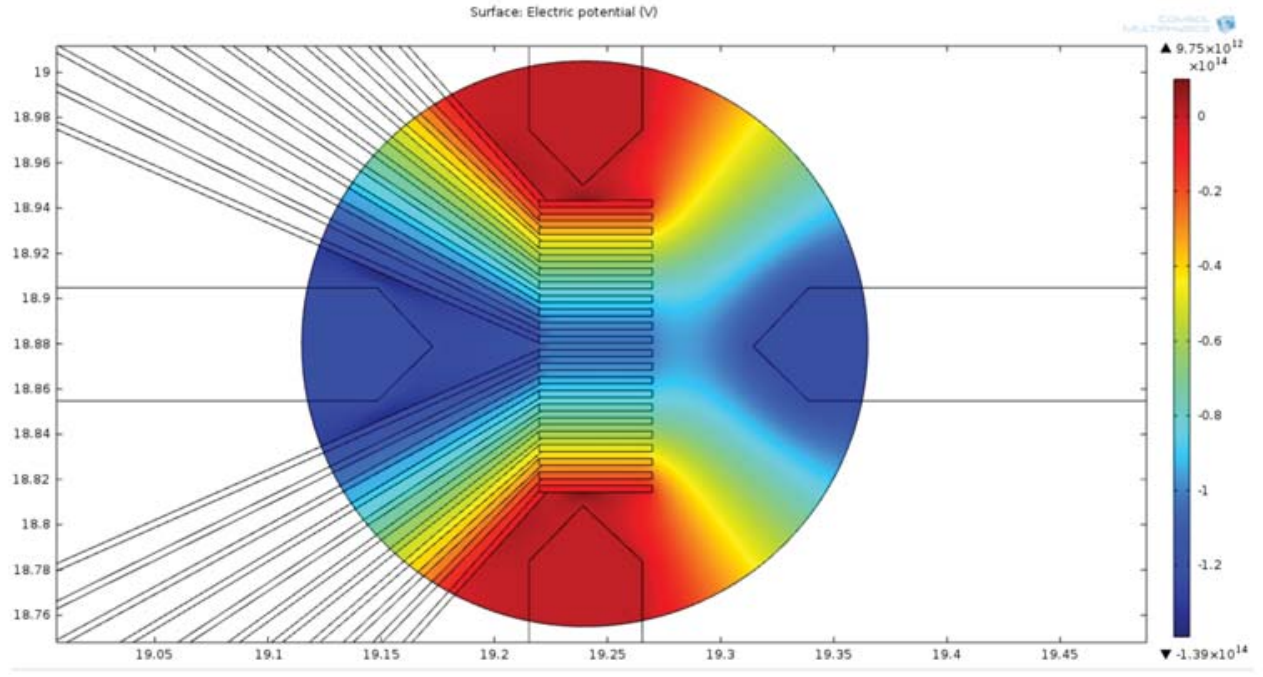

10

Figure 2.8: $2 \mathrm{D}$ simulation of electric potential over drive electrodes and sensing electrodes for $1 \mathrm{MHz}$ with $25 \mathrm{~nm}$ passivation done in collaboration with Jeana Collins

These are shown in Figure 2.14 and Figure 2.15 respectively. An effort was made to keep the ratio of the areas the same. In case of the $3 \mu \mathrm{m}$ gap circular array, this ratio was kept 1.25 , and it was 1.5 for the $7 \mu$ m gap array.

The motivation behind keeping the ratio of areas the same was that capacitance depends on area of the plates as well as the distance separating them. Since the design was constrained in a way that the area of electrodes could not be the same, the ratio was kept the same in order to ease modeling and to get a predictable response.

The metallization mask was made in such a way that it could be aligned over the 
- $0.1 \mathrm{MHz}$

- $5 \mathrm{Vpp}(-2.5$ to 2.5$)$

- Thin, low permittivity gap, $25 \mathrm{~nm}$

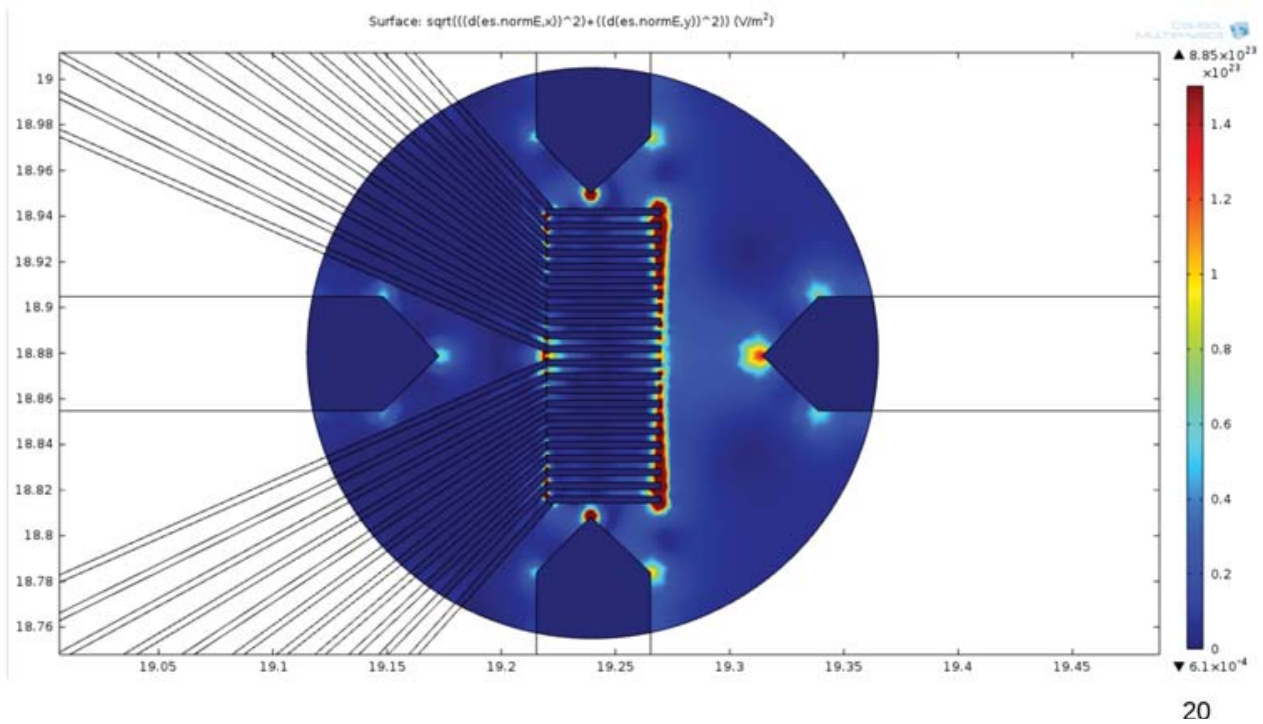

Figure 2.9: 2D simulation of electric potential over drive electrodes at $100 \mathrm{kHz}$ and sensing electrodes at $32 \mathrm{kHz}$ with $25 \mathrm{~nm}$ passivation done in collaboration with Jeana Collins

sensing layer. This contained the leads and contact pads, as well as two large triangular grounding pads to isolate the drive electrodes from the sensing electrodes. This mask is shown in Figure 2.16 and a closeup view of the die is shown in Figure 2.17.

An overlay of the metallization and the sensing layers is shown in Figure 2.18.

This design was considered adequate for initial testing of concept. However it had a number of problems experimentally, such as edge beading of photoresist, inability to find an edge connector of the specified pitch and thickness, non optimal quality of substrate etc. leading to process issues as well as non uniformity in testing. These processing issues are discussed in further depth in Chapter 3. Also, there as a need 
- $1.0 \mathrm{MHz}$

- $5 \mathrm{Vpp}(-2.5$ to 2.5$)$

- Thin, low permittivity gap, $25 \mathrm{~nm}$

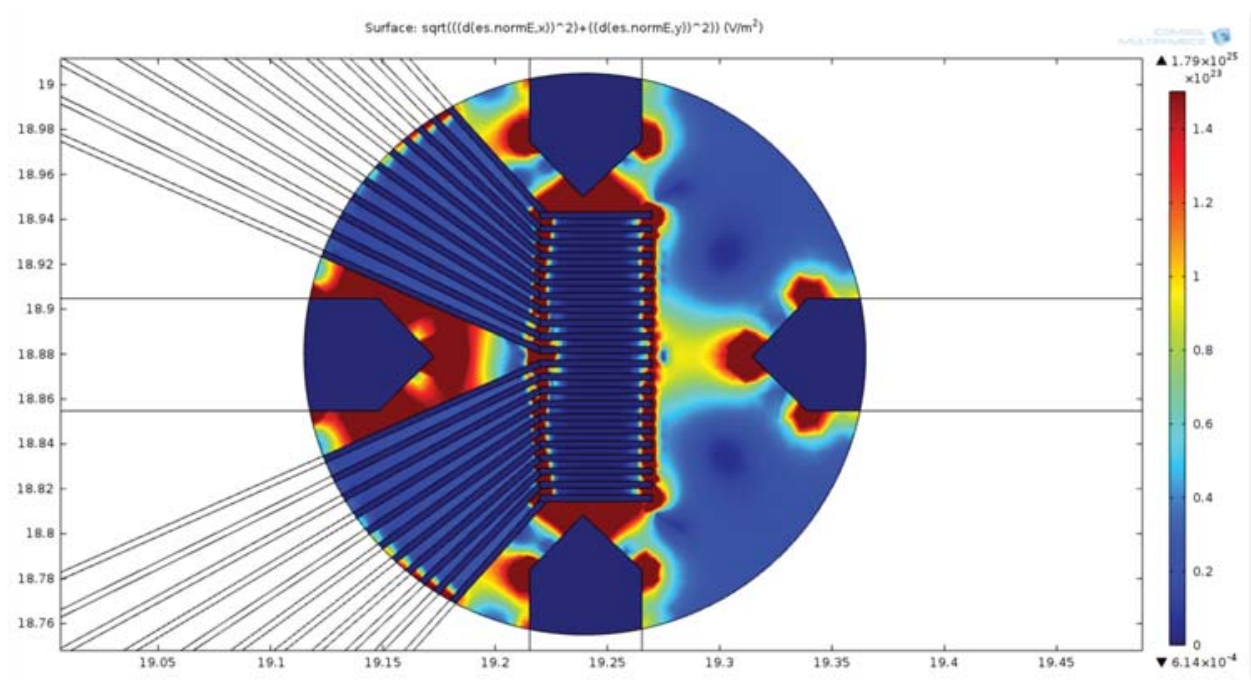

22

Figure 2.10: 2D simulation of electric potential over drive electrodes at $1 \mathrm{MHz}$ and sensing electrodes at $32 \mathrm{kHz}$ with $25 \mathrm{~nm}$ passivation done in collaboration with Jeana Collins

to increase the scale of production of these devices. Hence, a decision was taken to use 4" glass substrates instead of slides.

\subsubsection{Wafer Scale Design}

When the design was elevated to wafer scale, the four designs from slide scale processing were retained. Additionally, two more designs were added to isolate the nonadjacent sensing electrodes from a directly applied potential. This was essentially an altered design of the parallel plate electrode array with curved electrodes instead of linear rows. 


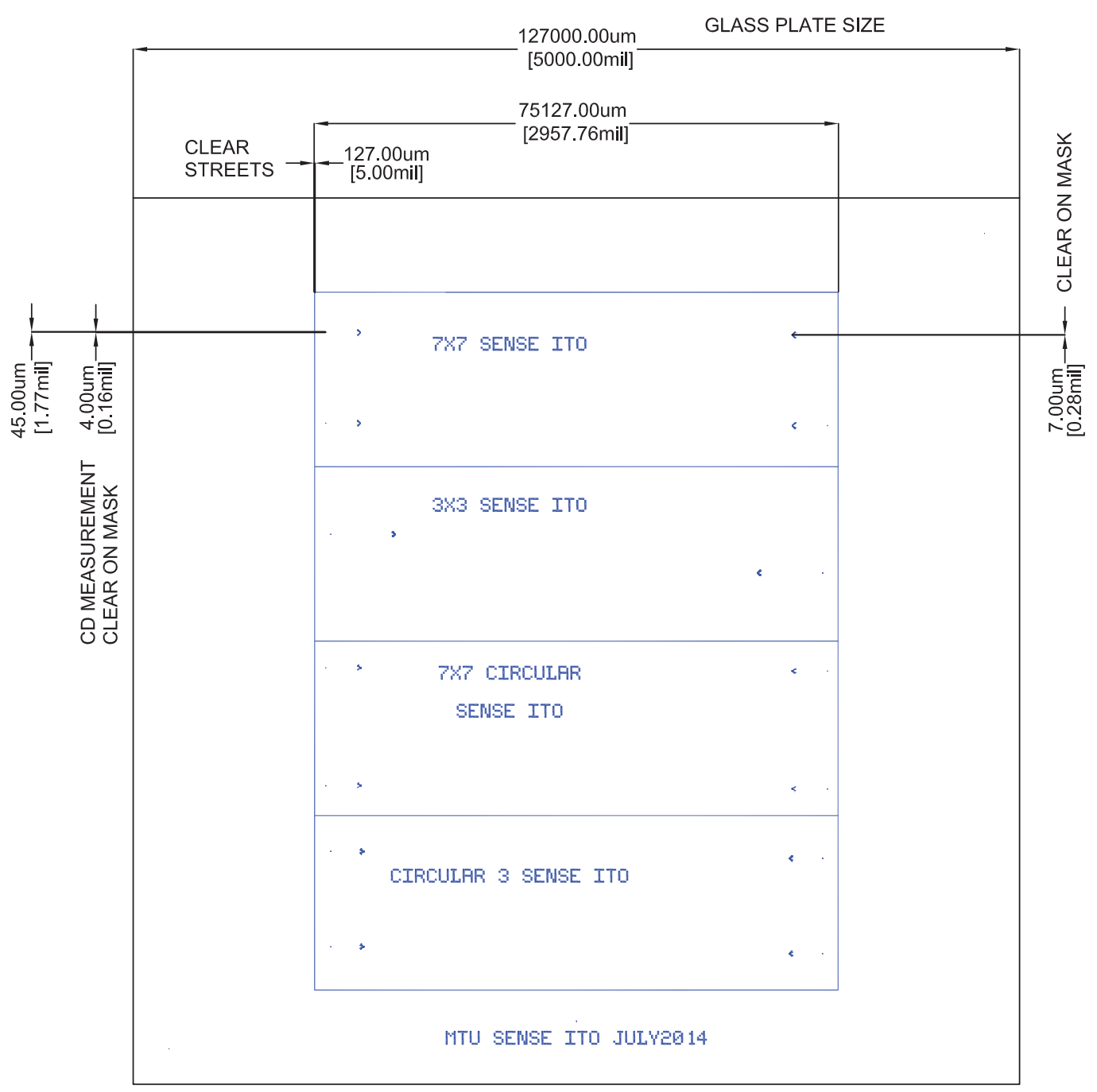

1. DIGITIZED DATA IS CLEAR, MASK FIELD IS DARK; (SOLID COLORED AREAS ON PLOTS WILL BE TRANSPARENT TO LIGHT ON MASK);

2. IMAGE VIEWED THROUGH GLASS SIDE, MASK IS RIGHT READING CHROME DOWN(RRD);

Figure 2.11: Proof plot of sensing electrode mask

For ease of identification of devices Figure 2.19 shows the naming scheme that was used. Different devices were randomly placed into a grid of rows and columns so as to ease troubleshooting of process issues based on position on wafer.

Figure 2.20 shows the design of the mask for sensing electrodes and placement of devices. 


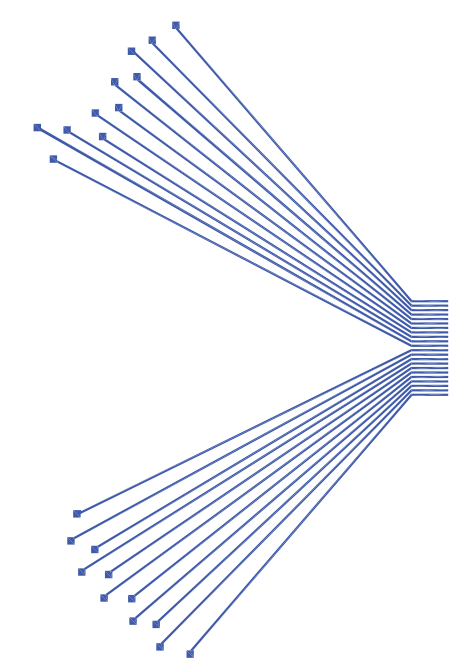

Figure 2.12: Proof plot of 3Linear sensing electrode

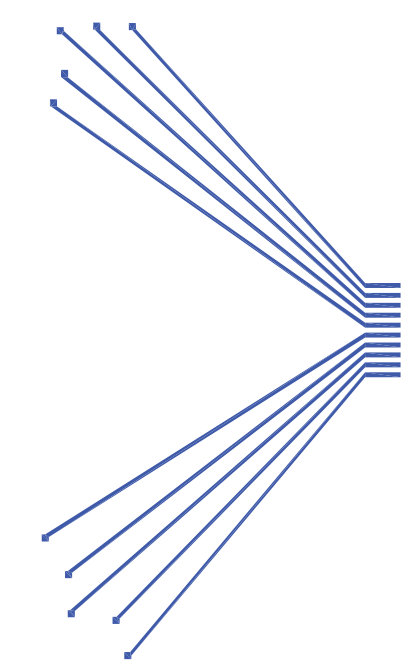

Figure 2.13: Proof plot of 7 Linear sensing electrode

Figure 2.21 shows the 3Linear design of electrodes which consisted of $3 \mu \mathrm{m}$ equal lines and spaces. Previous fabrication experience with the $3 \mu \mathrm{m}$ design on glass slides proved that the divergent lead pattern was hard to transfer since the CD was actually much smaller than $3 \mu \mathrm{m}$. Thus a decision was made to truncate the leads in such a way that the spacing was maintained as close to $3 \mu \mathrm{m}$ as possible. This is illustrated in Figure 2.22. 


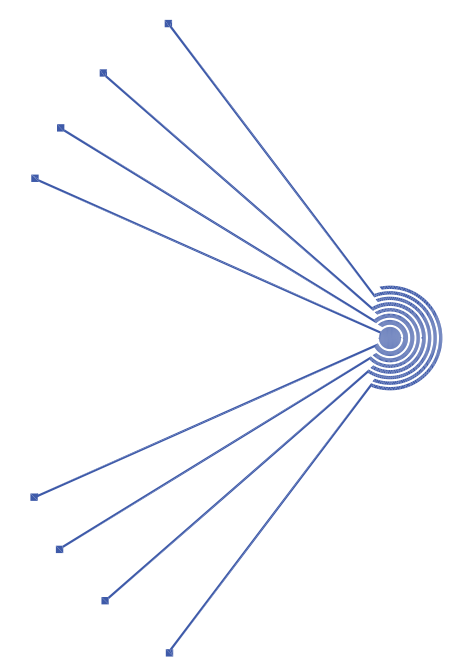

Figure 2.14: Proof plot of 3Circular sensing electrode

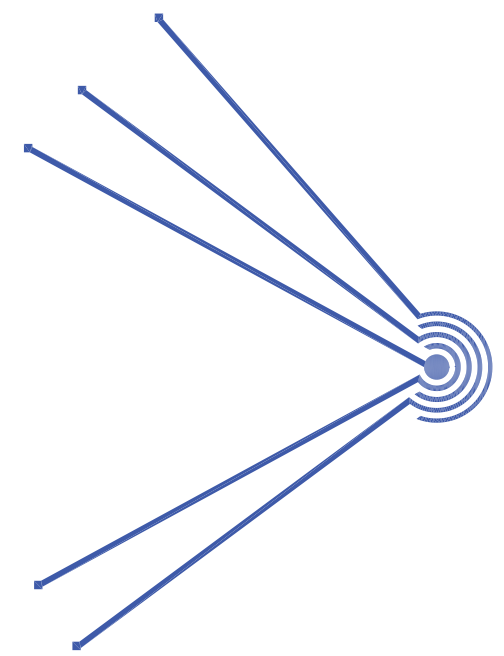

Figure 2.15: Proof plot of 7Circular sensing electrode 


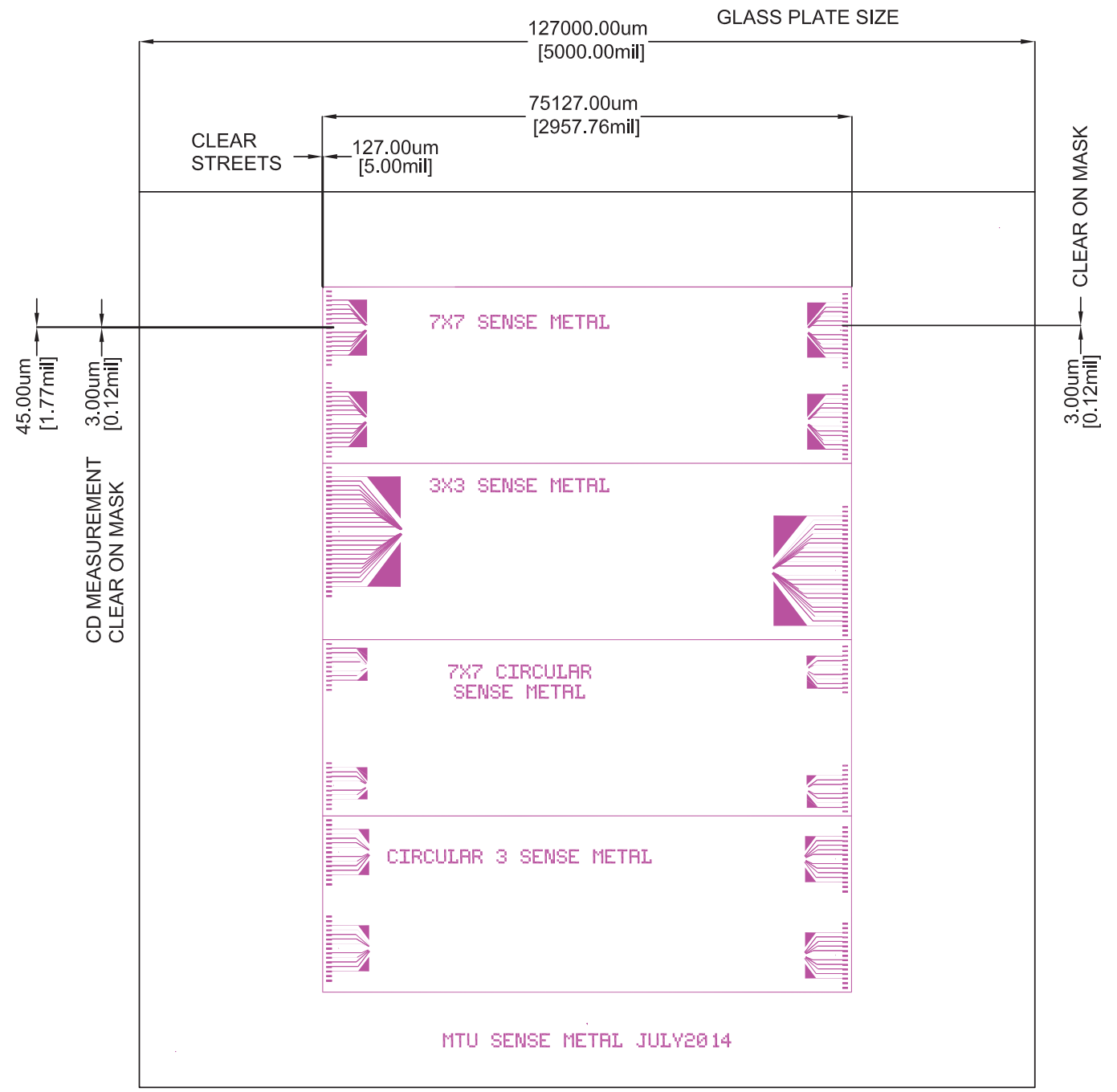

1. DIGITIZED DATA IS CLEAR, MASK FIELD IS DARK;

(SOLID COLORED AREAS ON PLOTS WILL BE TRANSPARENT TO LIGHT ON MASK);

2. IMAGE VIEWED THROUGH GLASS SIDE, MASK IS RIGHT READING CHROME DOWN(RRD);

Figure 2.16: Proof plot of metallization layer 


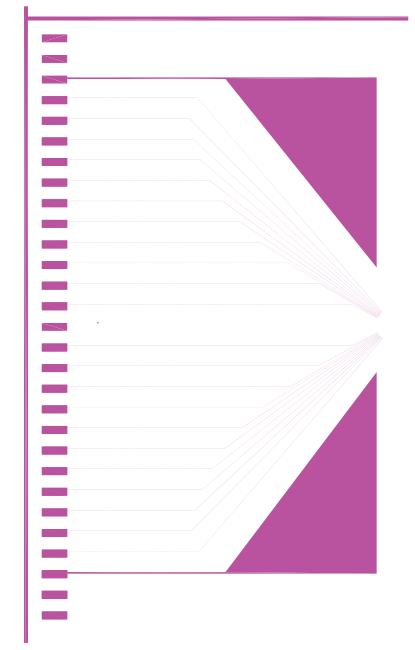

Figure 2.17: Proof plot of metallization die

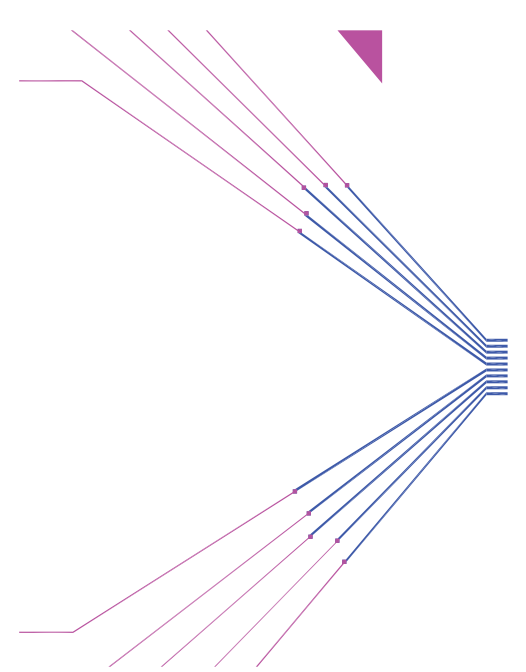

Figure 2.18: Proof plot of metallization layer overlaid on sensing electrode layer 


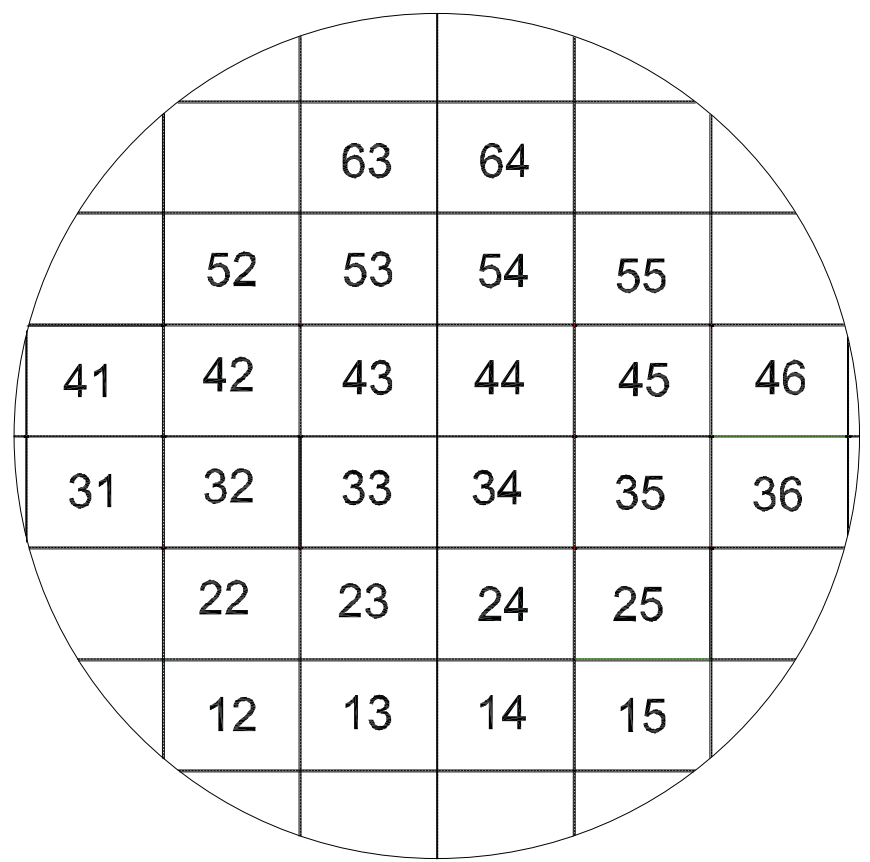

Figure 2.19: Device referencing scheme for wafer scale processing 


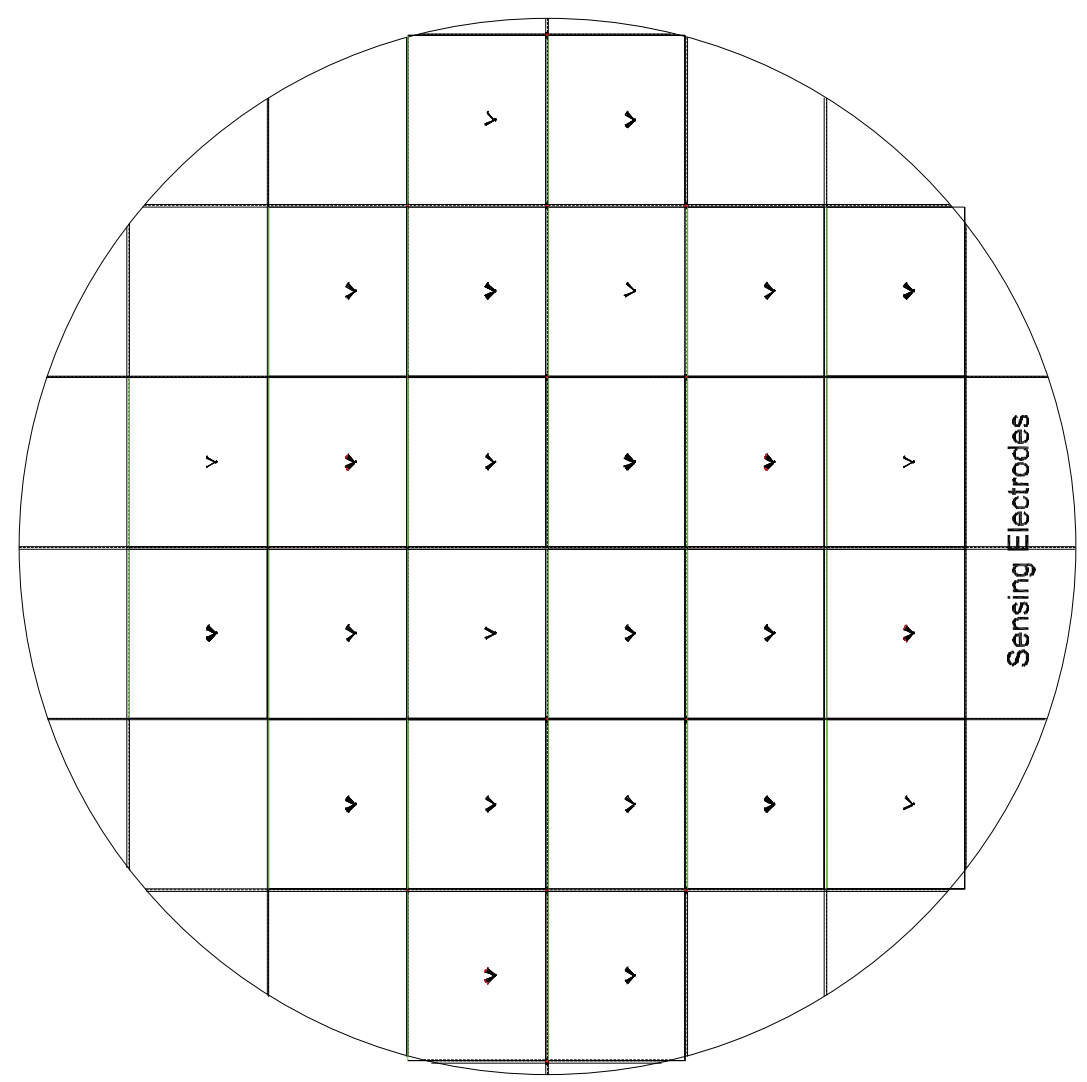

Figure 2.20: Layout of mask for sensing electrodes

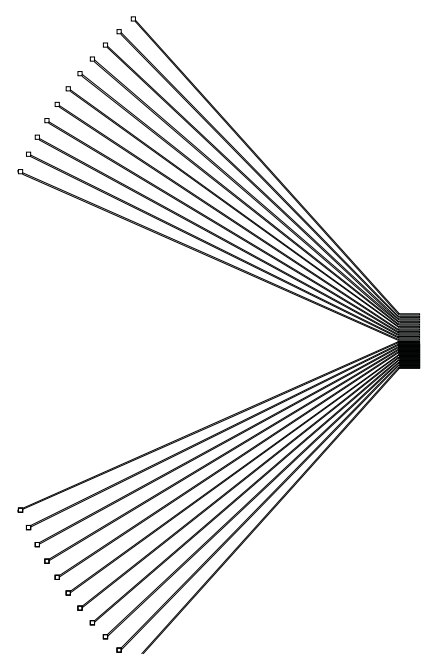

Figure 2.21: 3Linear design of sensing electrodes 


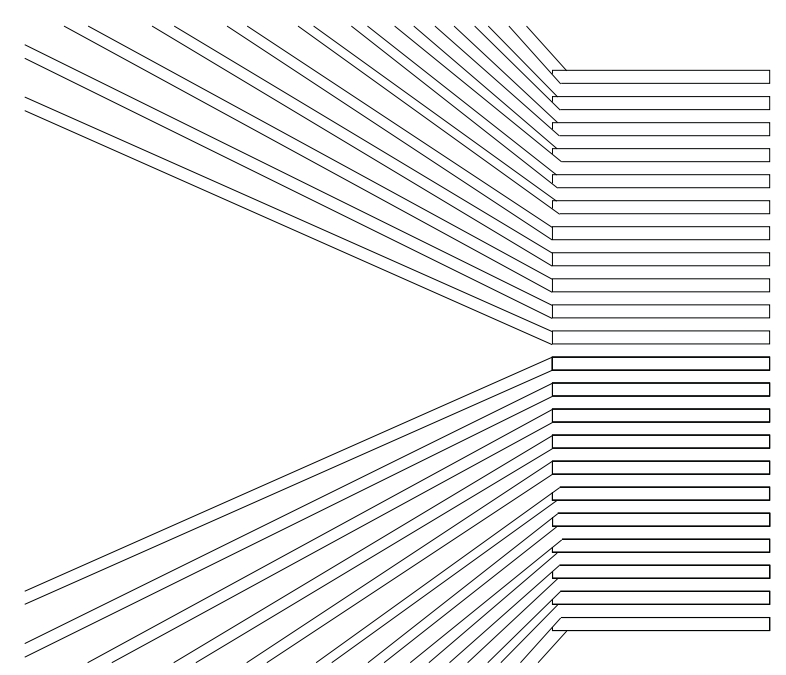

Figure 2.22: 3 Linear design of sensing electrodes showing truncated leads 
The 7Linear design of sensing electrodes was retained from slide scale processing. However, the divergent leads were equally spaced from one another. This is shown in Figure 2.23.

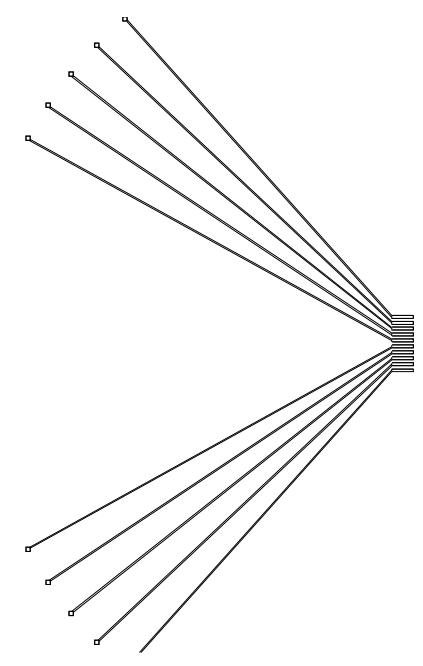

Figure 2.23: 7Linear design of sensing electrodes

The 3Circular design was retained. However, the circular leads were truncated to lower interference and the divergent leads were placed equidistant from one another. This is shown in Figure 2.24 and more clearly in Figure 2.25.

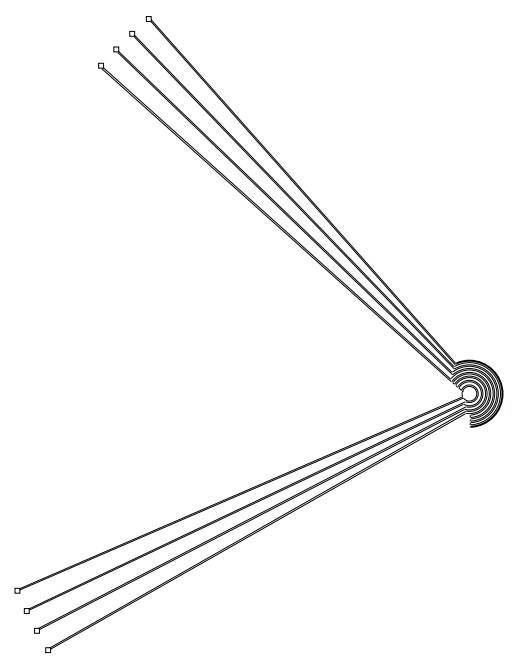

Figure 2.24: 3Circular design of sensing electrodes 


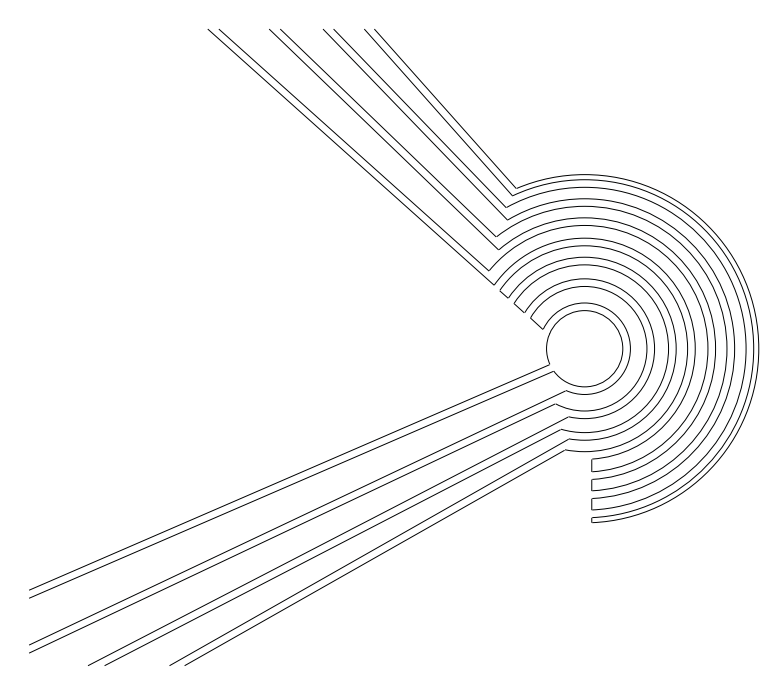

Figure 2.25: Close up of 3 Circular electrode showing $3 \mu \mathrm{m}$ spacing of leads 
The design for 7Circular sensing electrodes was retained as well, and this is shown in

Figures 2.26 and 2.27 .

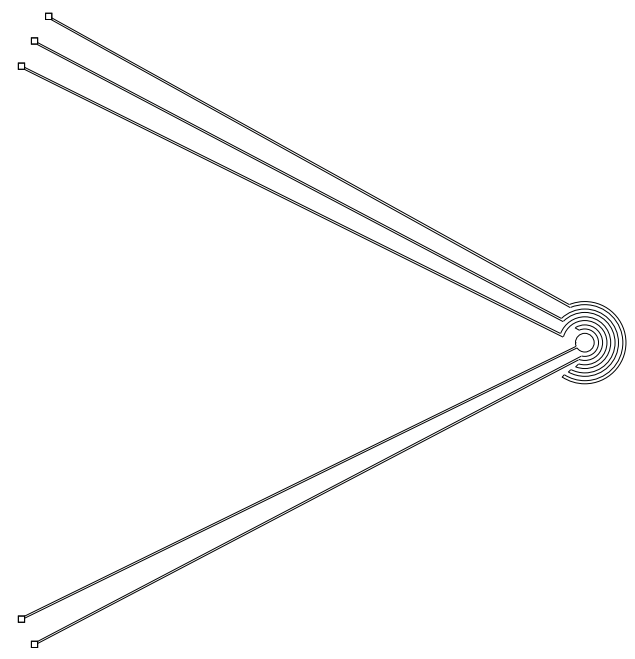

Figure 2.26: 7Circular design of sensing electrodes

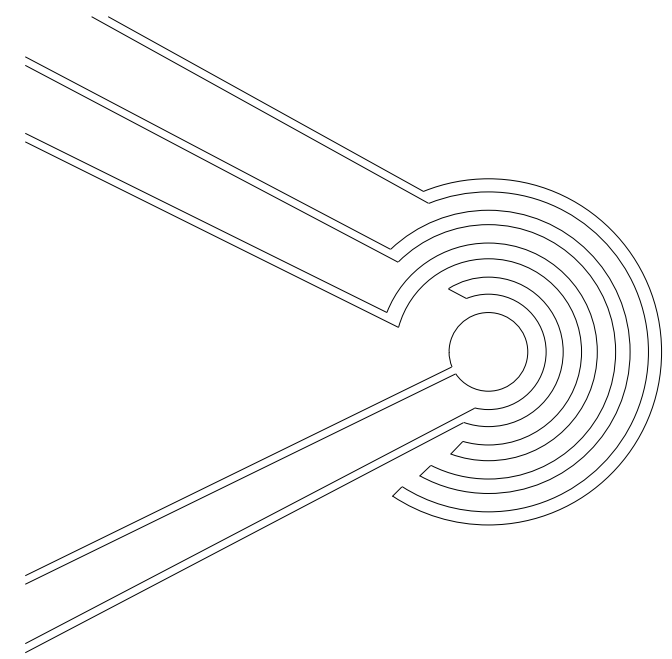

Figure 2.27: Close up of 7 Circular electrode showing $7 \mu \mathrm{m}$ spacing of leads

A new design of curved electrodes was proposed for the sensing layer. In this design, adjacent leads could be used as parallel plates since the circular design did not encompass the entire area which could be under the influence of the field from drive electrodes. This design was made to account for $3 \mu \mathrm{m}$ (Figure 2.28) and $7 \mu \mathrm{m}$ spaces 
(Figure 2.29).

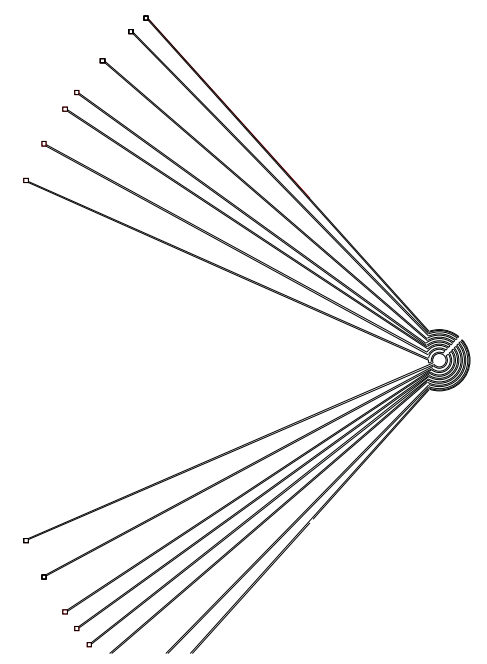

Figure 2.28: 3Curved design of sensing electrodes 


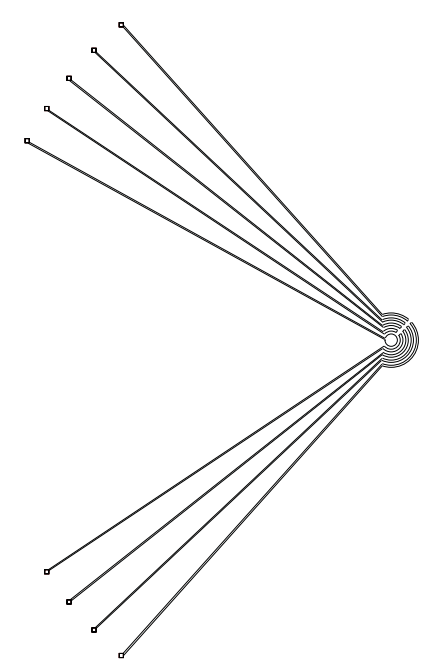

Figure 2.29: 7Curved design of sensing electrodes

One of the biggest challenges faced during fabrication was the alignment of metallization layer to the sensing electrode layer. To ease this, alignment marks were put in scribe lines between the dies. These alignment marks were all labeled according to their position to ensure correct placement. The alignment mark on sensing electrode layer is shown in Figure 2.30.

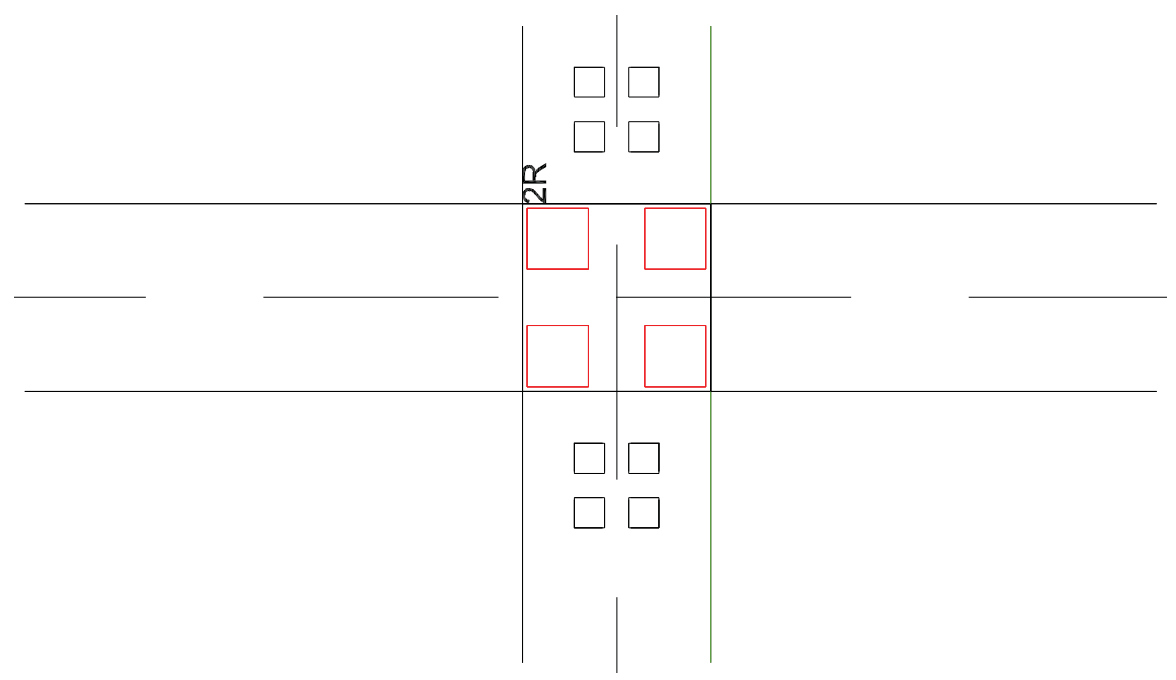

Figure 2.30: Alignment marks on sensing electrode mask 
The metallization layer (Figure 2.31) was designed in such a way that all the dies were exactly the same. Each lead ended in $10 \mu \mathrm{m} \times 10 \mu \mathrm{m}$ contact pads (Figure 2.32) which would be placed over similar $10 \mu \mathrm{m}$ x $10 \mu \mathrm{m}$ contact pads on the sensing layer. This was done to ensure good contact between sensing and metallization layers.

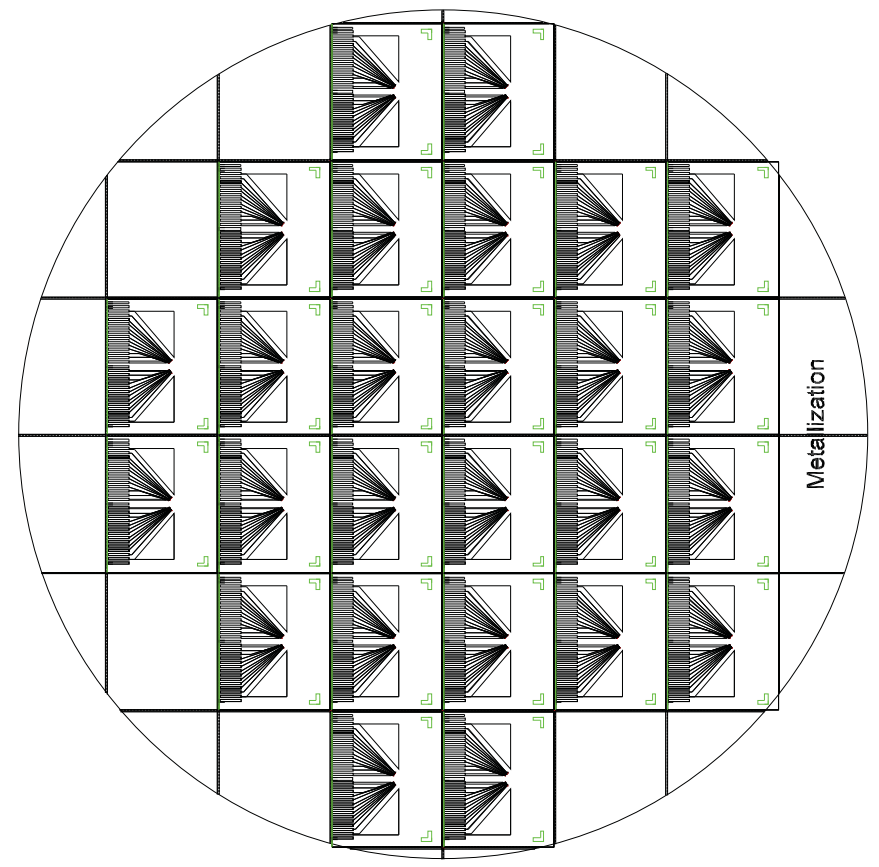

Figure 2.31: Metallization layer mask
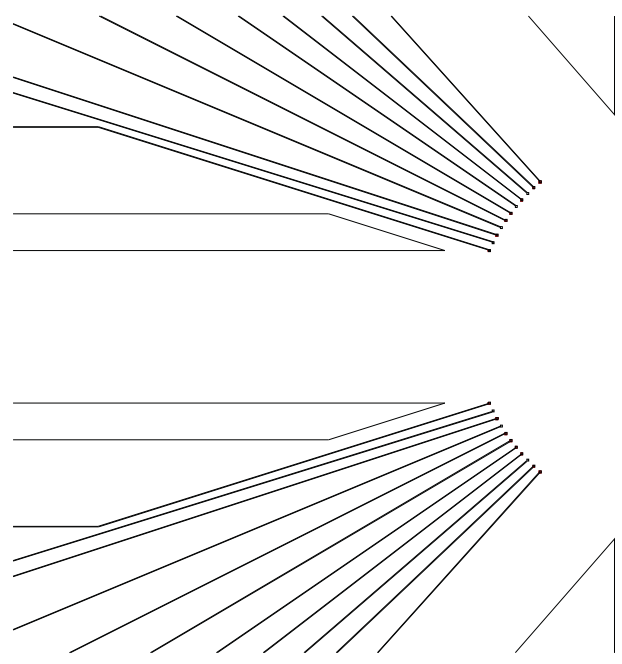

Figure 2.32: Contact areas for aligning sensing layer to metallization layer 
All the dies were made similar (Figure 2.33) to ensure flexibility in design for sensing electrodes. The design for sensing electrodes was still tentative and needed testing to determine if it could be characterized easily. Hence, in case of change of design of sensing electrodes, the metallization mask could still be used.

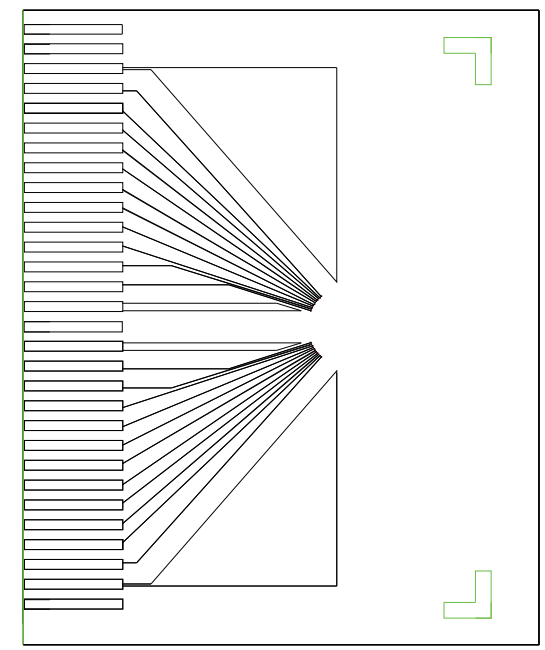

Figure 2.33: Close up view of metallization layer showing a die 
Labelled alignment marks were placed in the scribe lines. This is shown in Figure 2.34

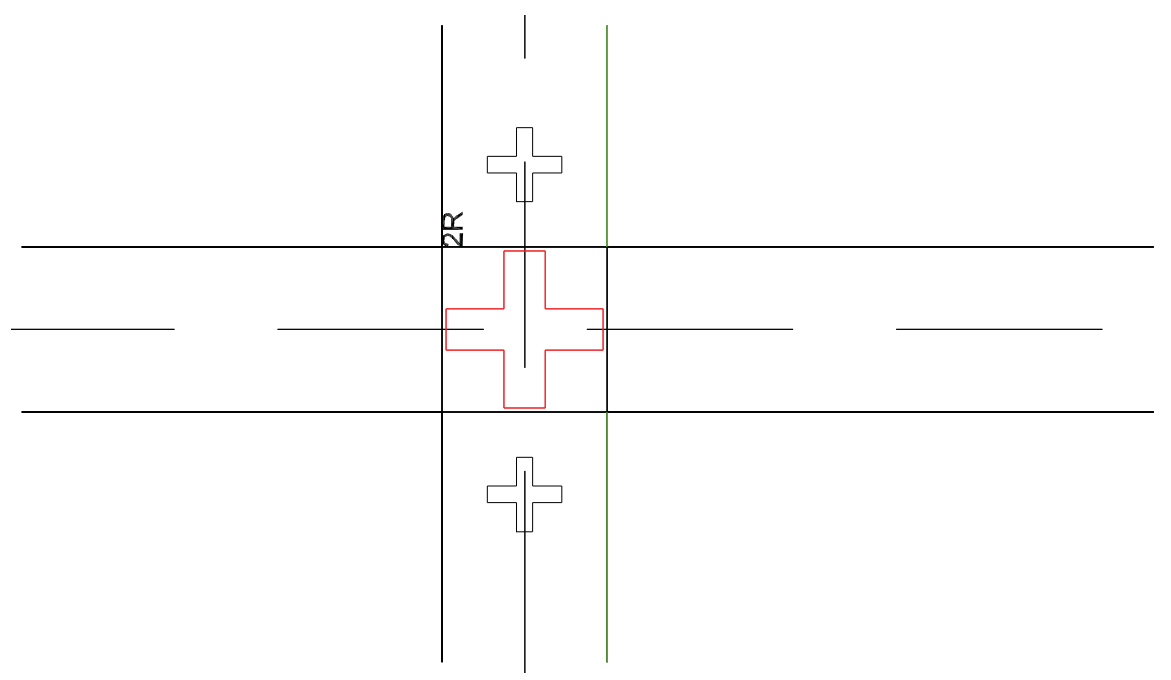

Figure 2.34: Alignment marks on metallization mask 
The design was reviewed and verified by overlaying the designs of both layers. This is shown in Figures 2.35, 2.36 and 2.37.

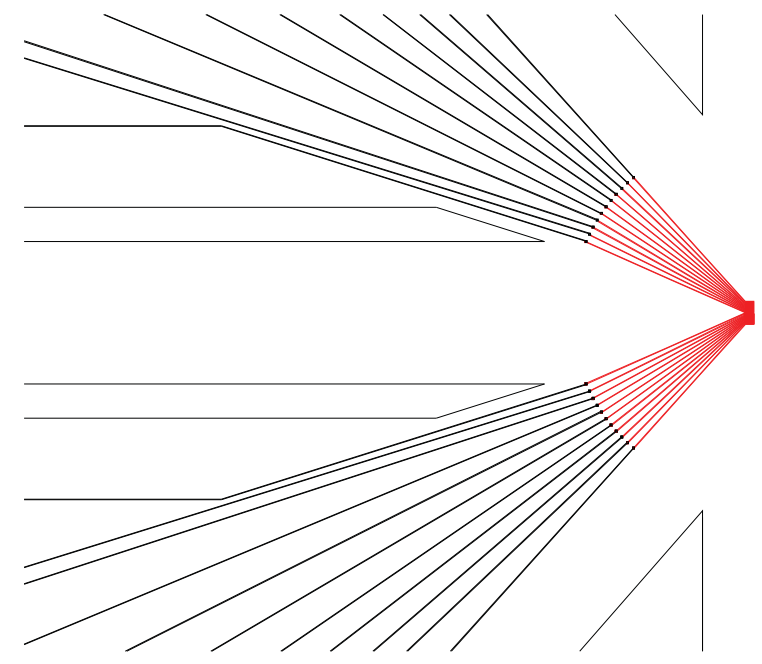

Figure 2.35: Alignment of sensing electrodes to metallization layer in 3Linear devices

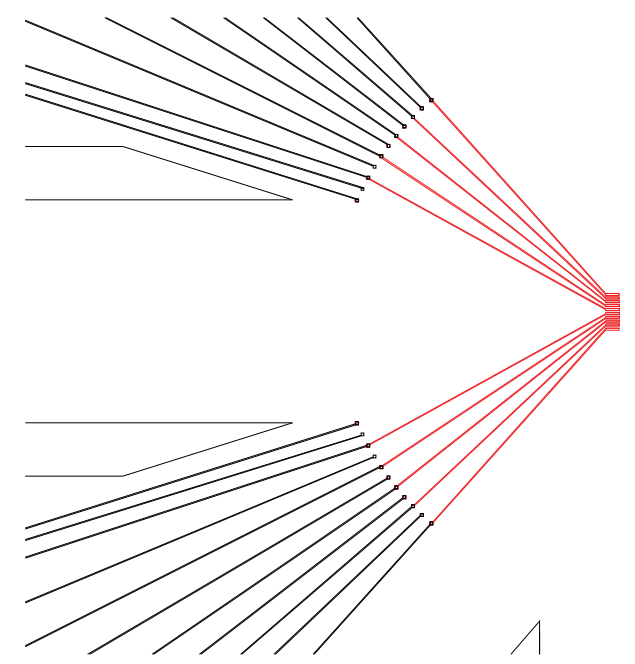

Figure 2.36: Alignment of sensing electrodes to metallization la0yer in 7Linear devices 


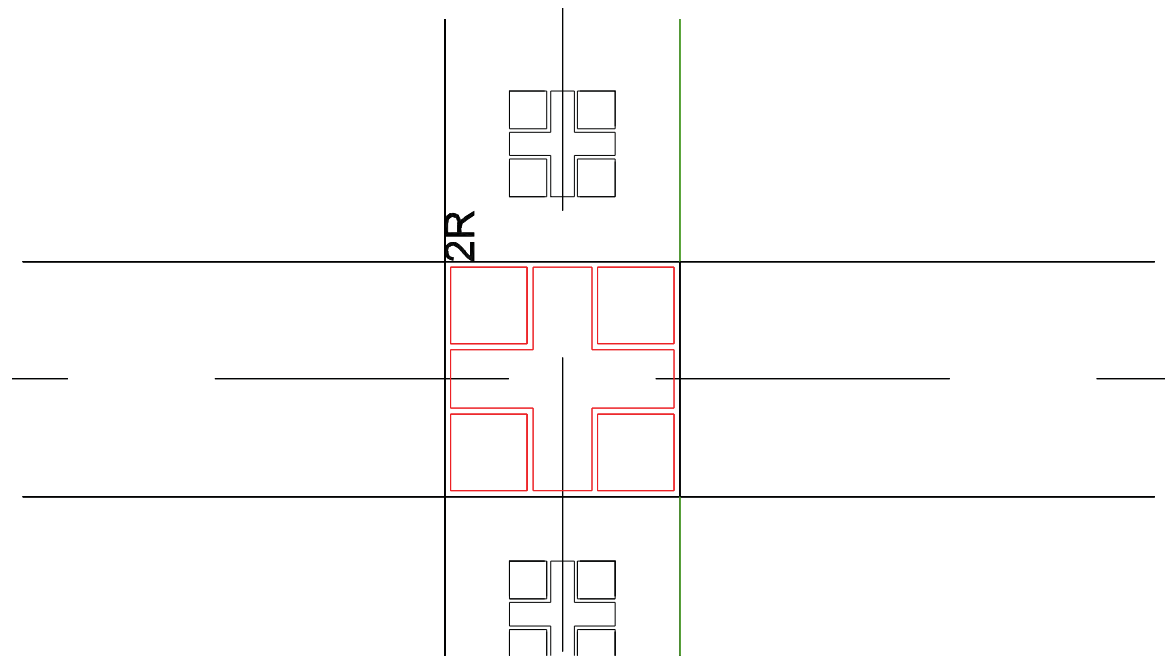

Figure 2.37: Alignment marks of metallization layer over sensing layer 
Drive electrodes (Figure 2.38 and 2.39) to generate the ac field required for cell positioning were designed as quadruple points (Figure 2.40). Two of which these were active and the other two were held at zero potential. This design was based on previous research [22].

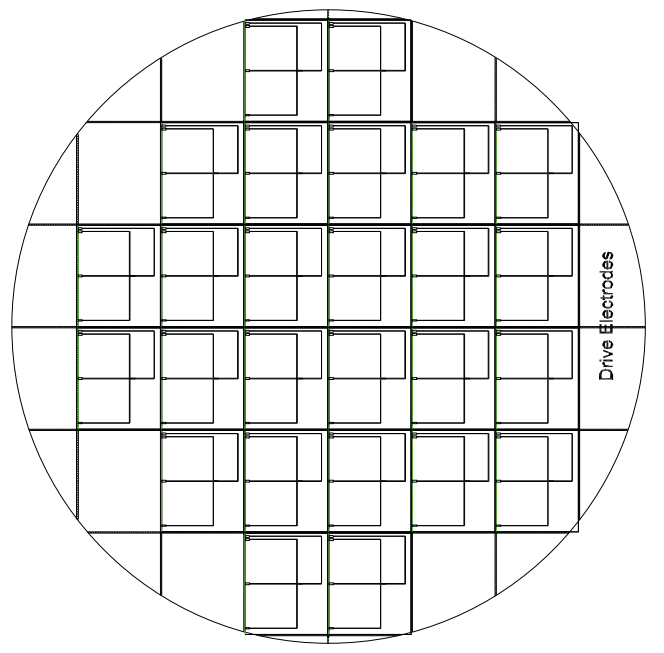

Figure 2.38: Drive electrode mask

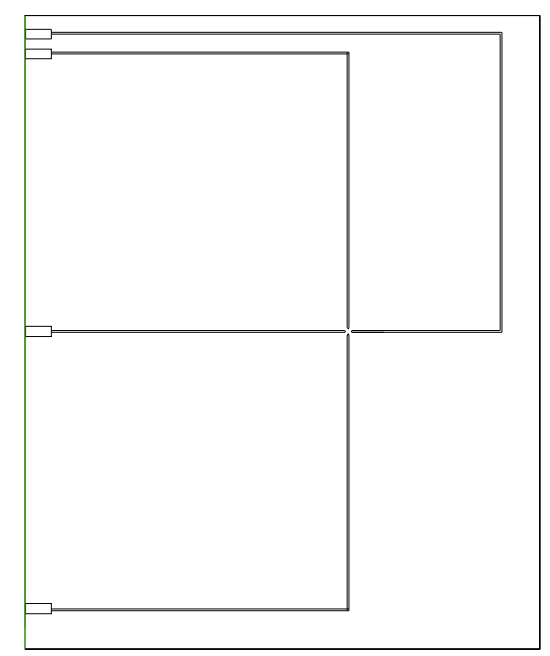

Figure 2.39: Close up of drive electrode layer showing a die 


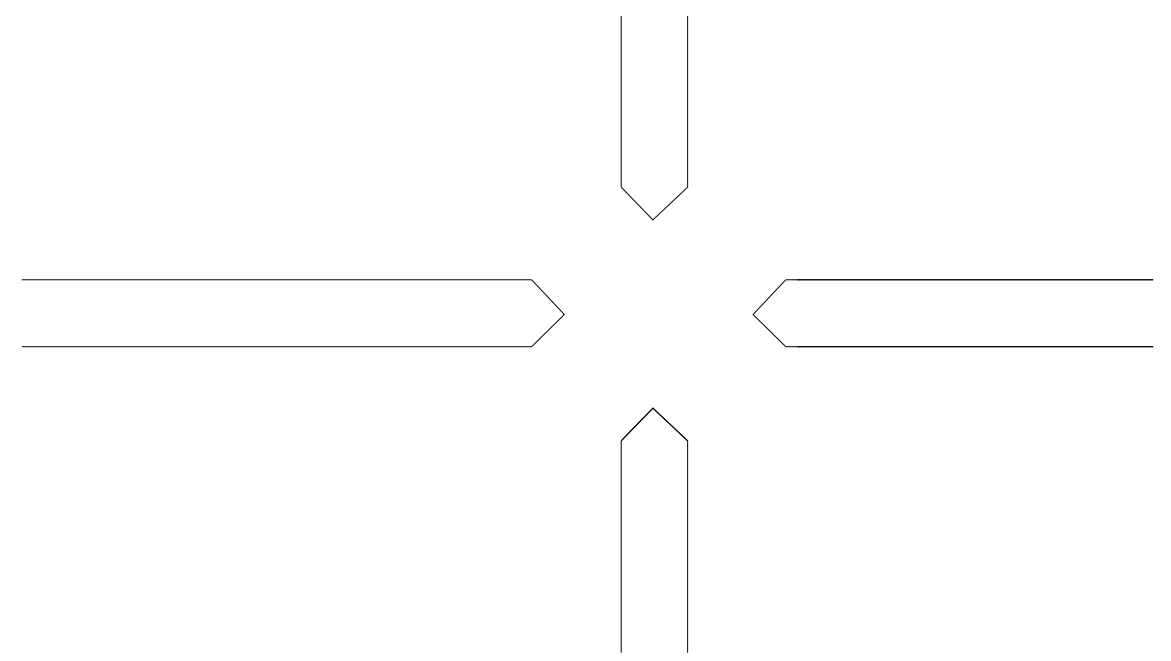

Figure 2.40: Quadruple drive electrodes 
Alignment marks (Figure 2.41) were placed in a similar fashion as described for sensing and metallization layers.

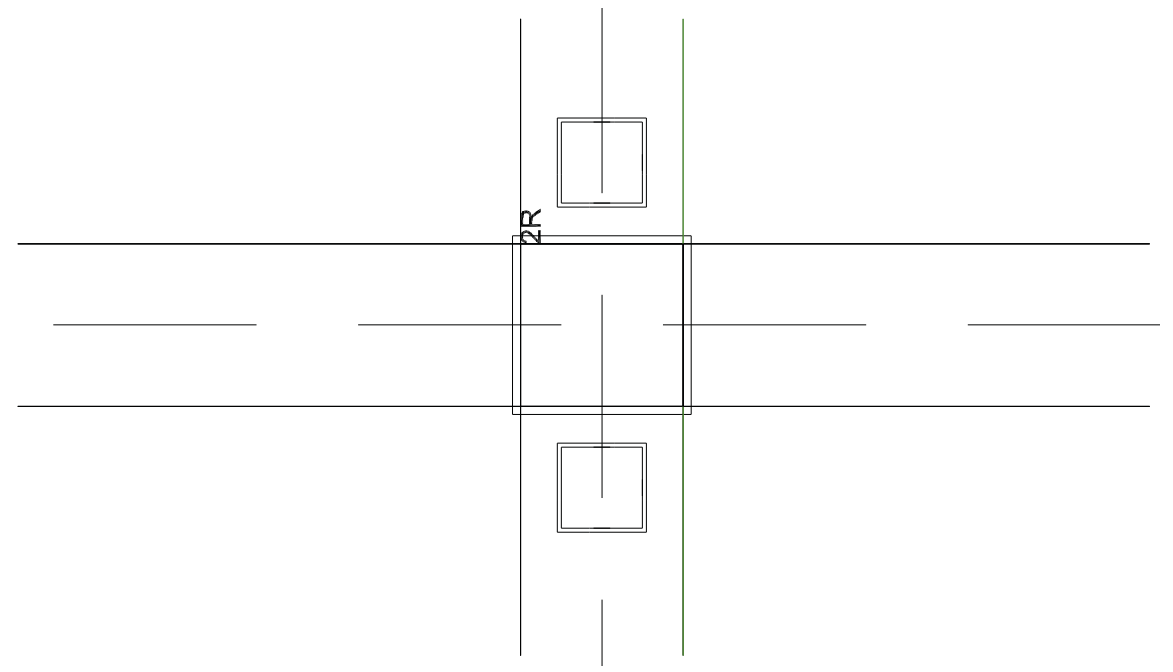

Figure 2.41: Alignment marks on drive electrode layer

A few overlaid designs are shown in Figures 2.42, 2.43 and 2.44.

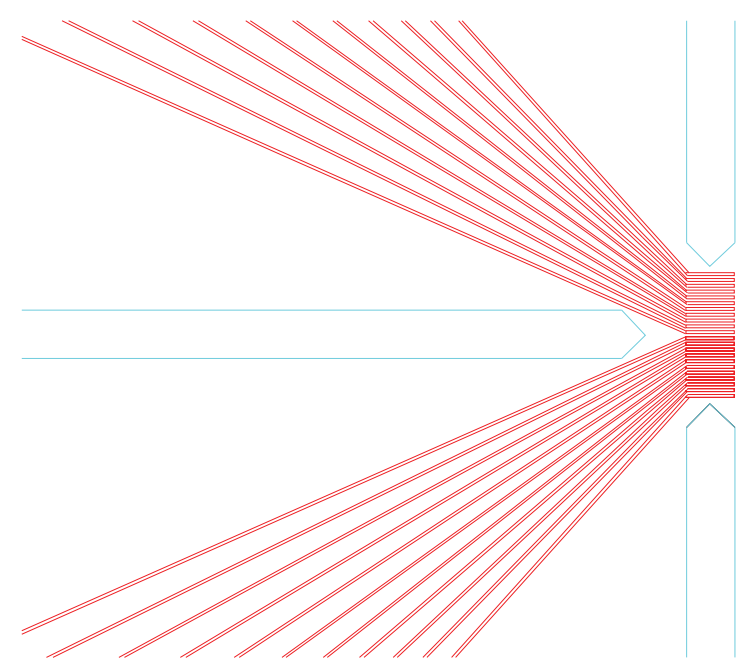

Figure 2.42: Alignment of drive layer to 3Linear sensing electrodes showing spacing and placement 


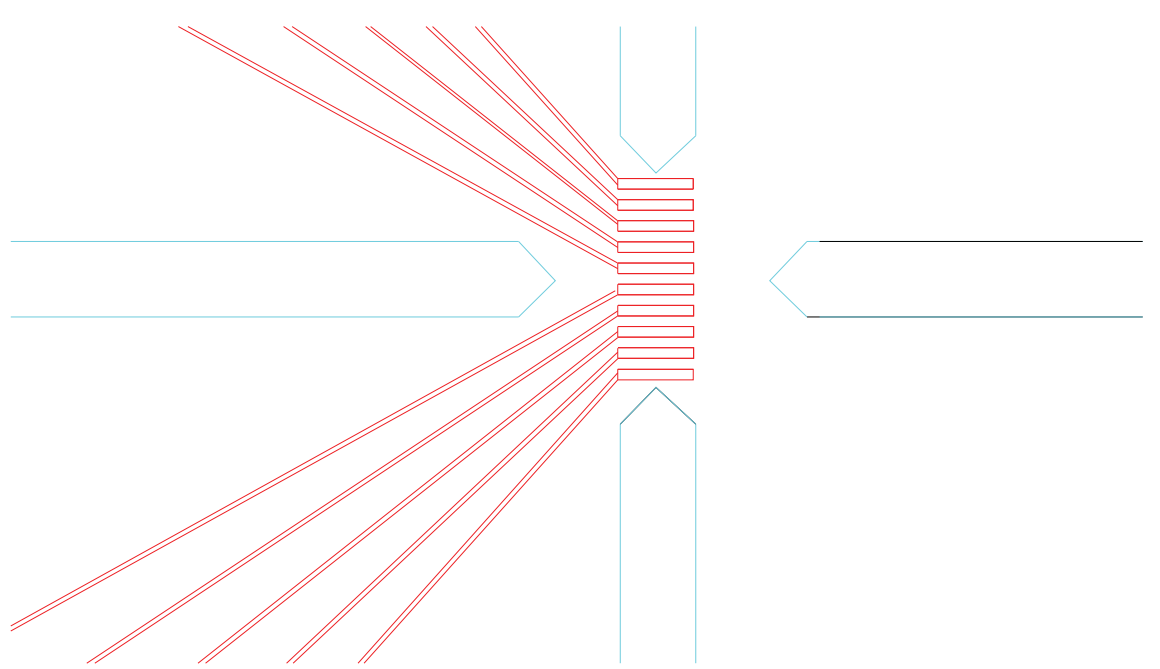

Figure 2.43: Alignment of drive layer to 3Linear sensing electrodes showing spacing and placement 


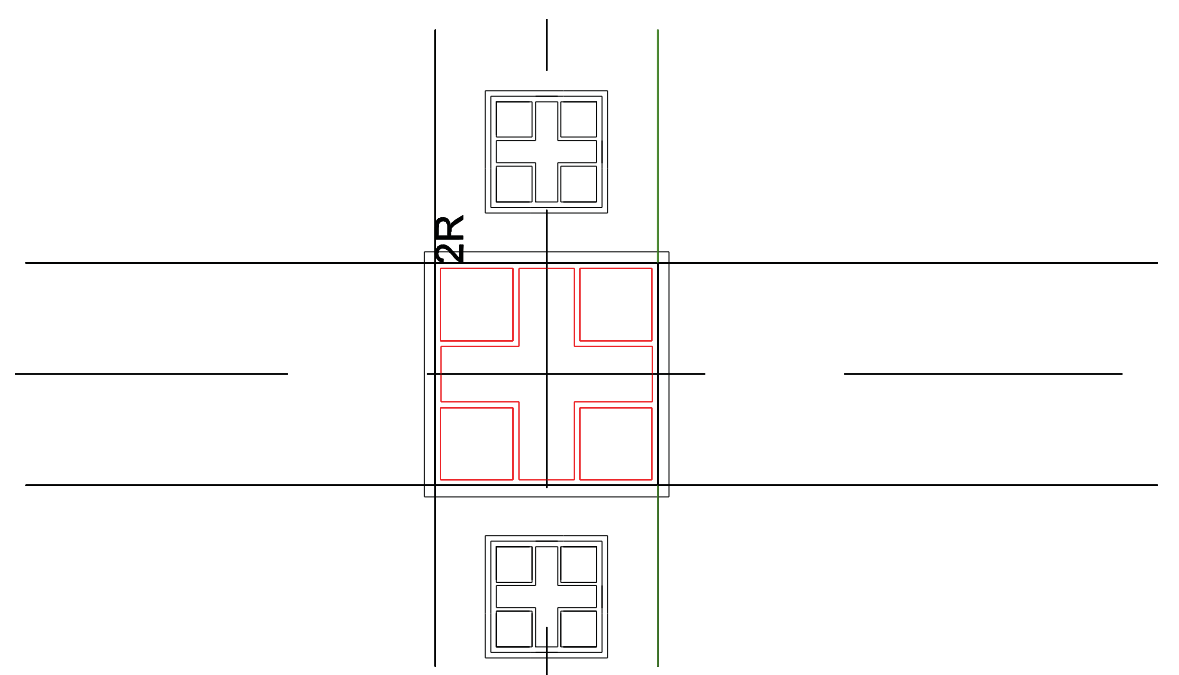

Figure 2.44: Alignment marks on sensing, metallization and drive layers

\subsubsection{Microfluidic Chamber}

A fluidic chamber was designed having a diameter of $1 \mathrm{~mm}$ (Figure 2.45) in accordance with the previously used design. This fluidic chamber had inlet and outlet ports defined for injecting test fluids using a syringe. This is shown in Figure 2.46.

The inlet and outlet ports here were at an angle to each other. This prevented the fluid from filling up the chamber completely, leading to air pockets that impeded detection of cells and polystyrene beads. Thus, an alternate design was constructed to ensure that the problem of air pockets was mitigated. The new design for wafer scale processing, as shown in Figures 2.47, 2.48 and 2.49 consisted of the channels exiting a $250 \mu \mathrm{m}$ wide chamber at an angle of exactly 180 degrees. 


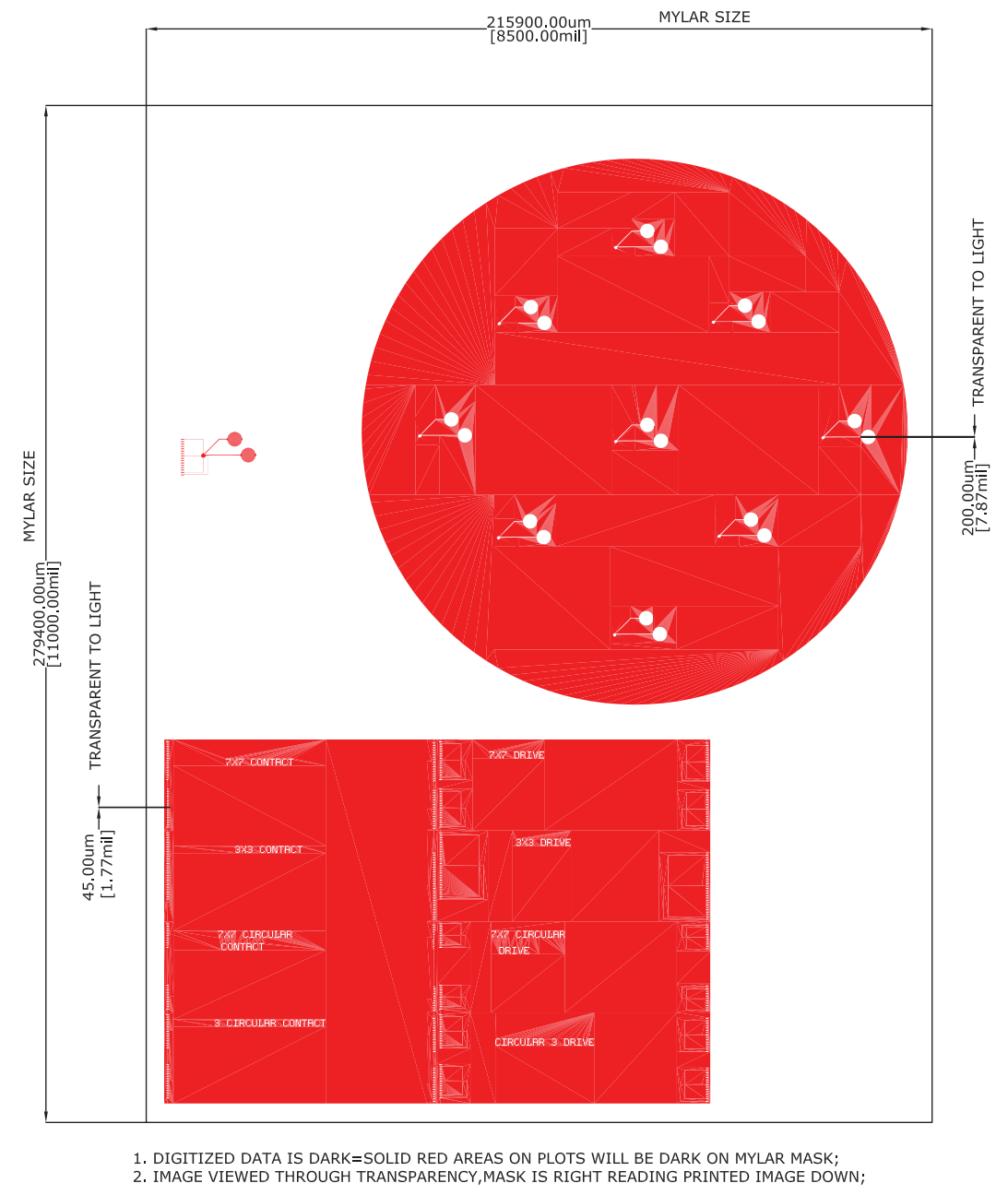

Figure 2.45: Proof plot of fluidic chamber 


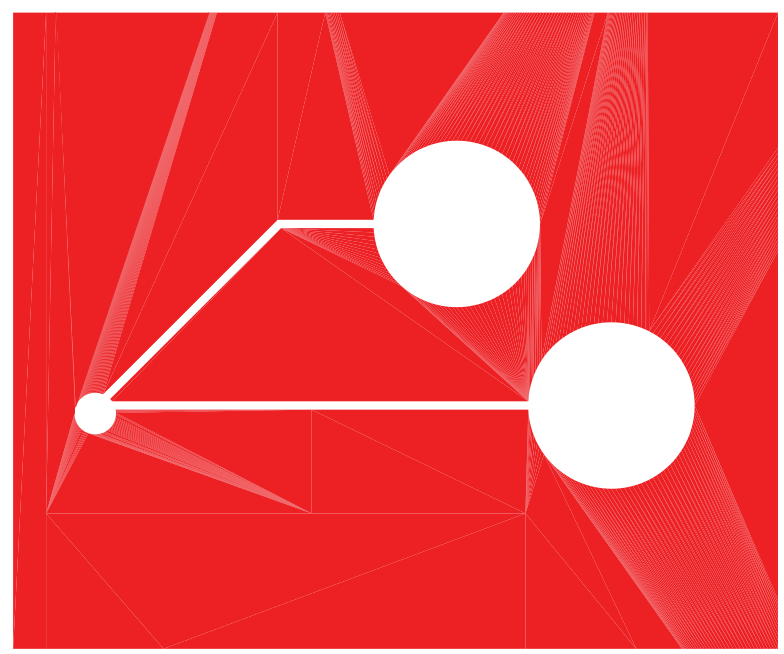

Figure 2.46: Proof plot of fluidic chamber showing $1 \mathrm{~mm}$ chamber, inlet and outlet ports

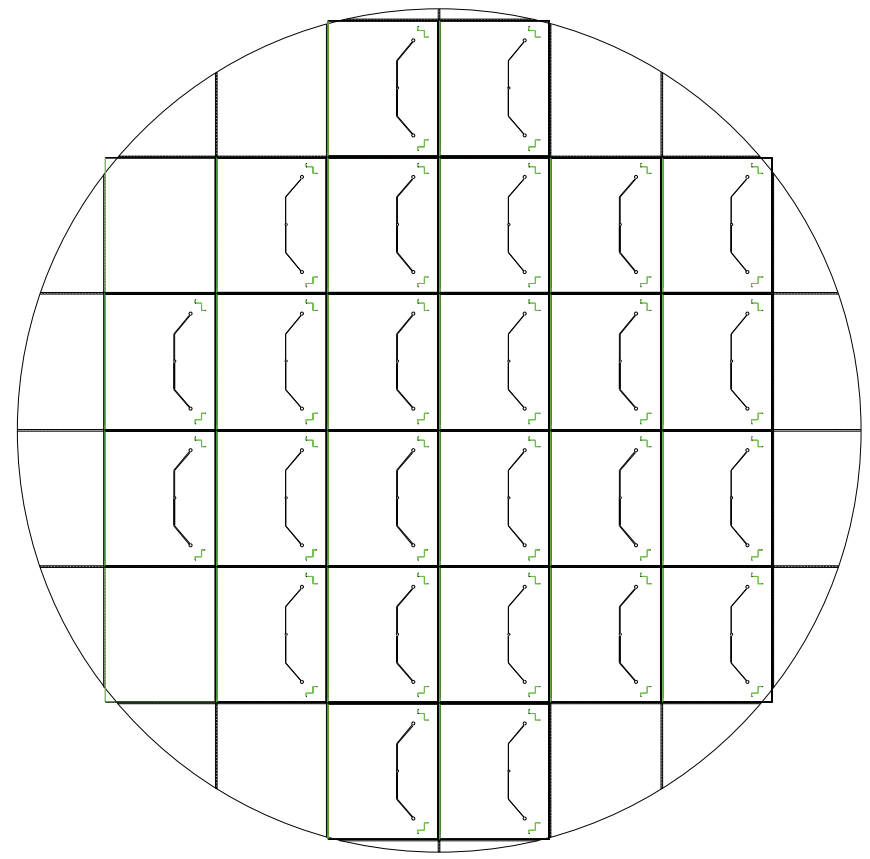

Figure 2.47: Design of microfluidic chamber mask 


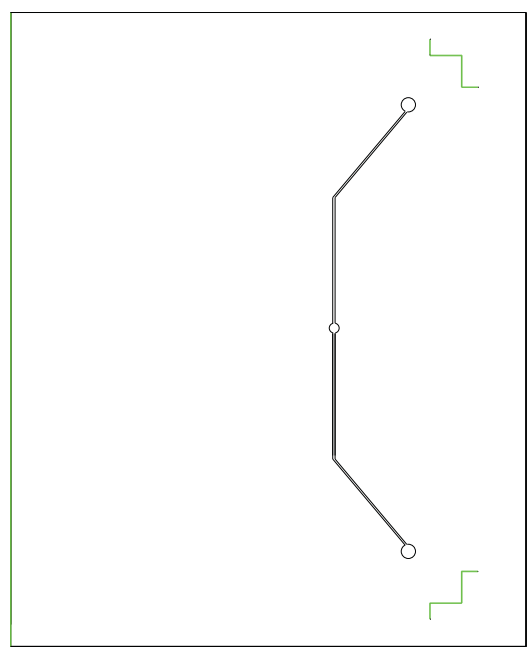

Figure 2.48: Close up of chamber layer showing a die 


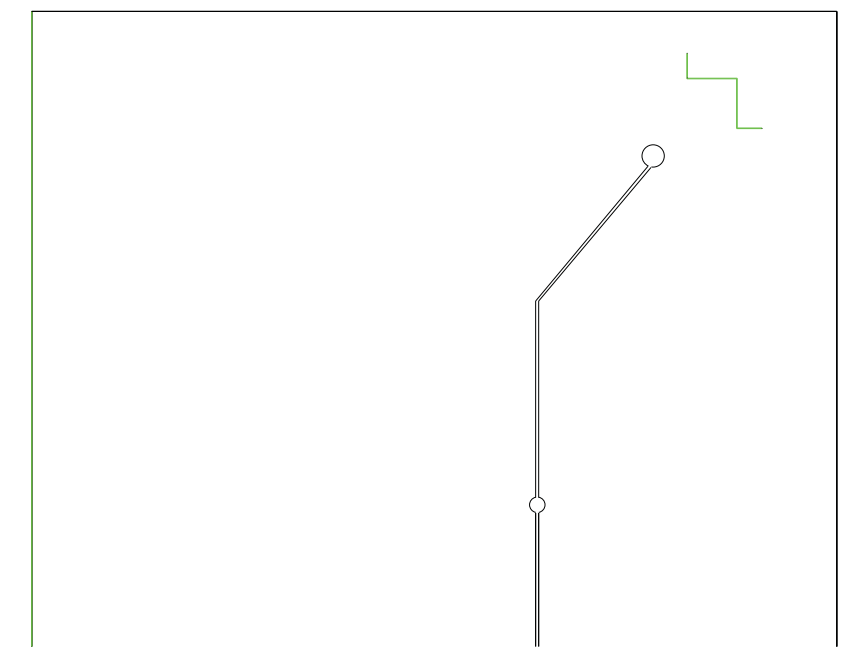

Figure 2.49: Close up of chamber layer showing microfluidic chamber and inlet port

Overlays showing placement of all the four layers are shown in Figures 2.50 and 2.51.

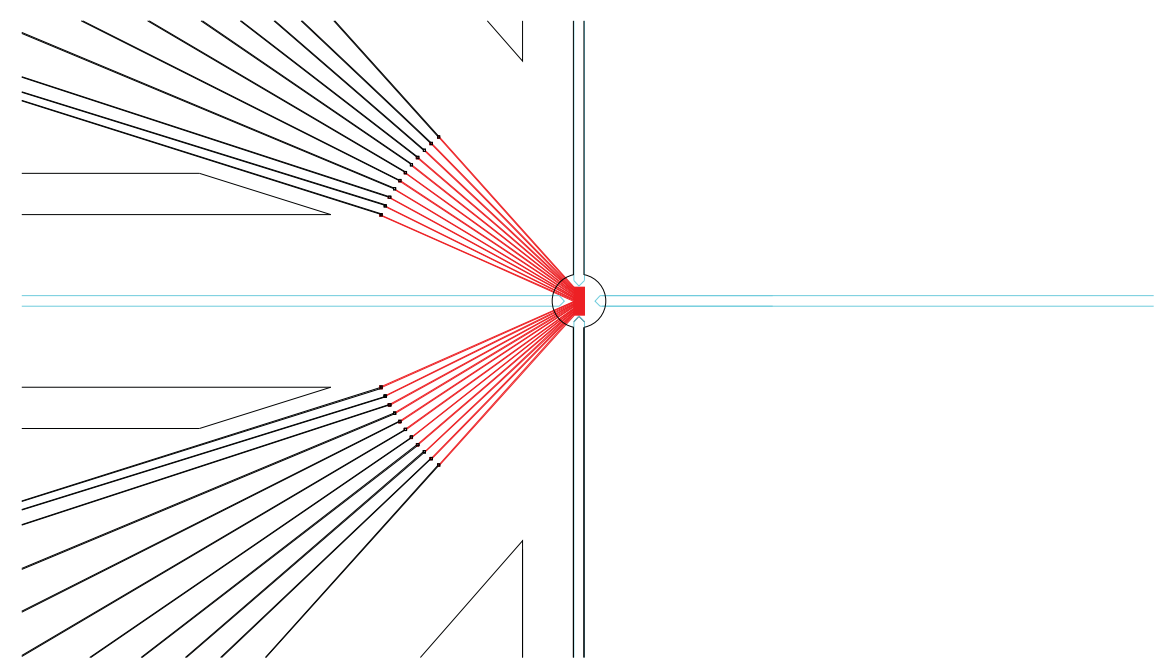

Figure 2.50: Overlay of sensing electrode, metallization, drive and chamber layers 


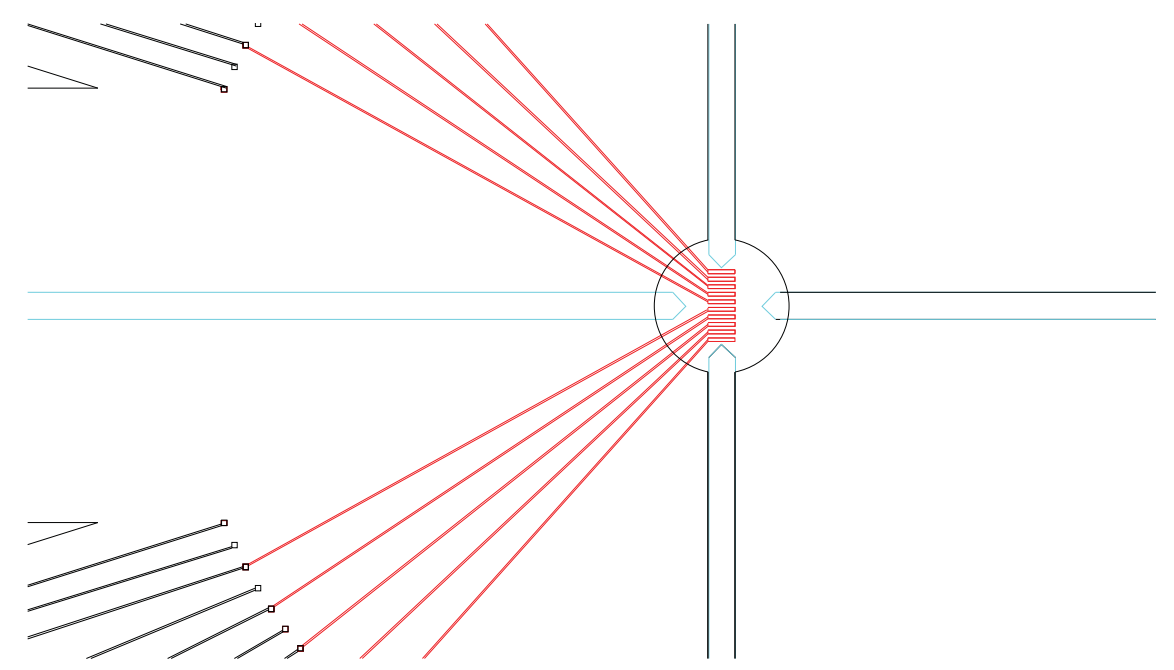

Figure 2.51: Close up of device masks

\subsubsection{Edge Connector SM3ZS067U310ABR120}

These devices were placed close to the edge of the glass slides in order to connect them to card edge connector SM3ZS067U310ABR120 obtained from JAE Electronics. This edge connector had a pitch of $500 \mu \mathrm{m}$ and 30 pins on one side of the notch. An Eagle drawing is shown in Figure 2.52. The die was designed in such a way that the pads were located close to the edge for ease of use with the connector, which was further connected to external circuitry. 


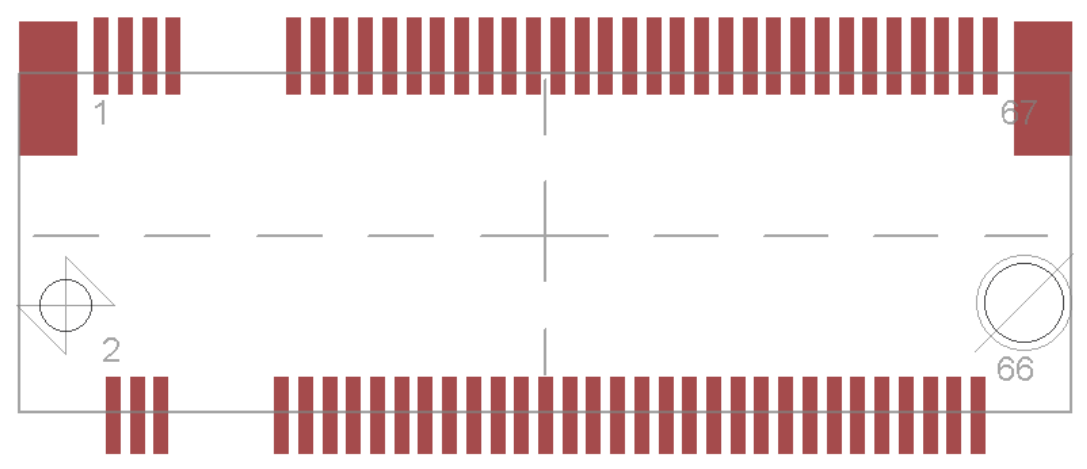

Figure 2.52: Eagle drawing of card edge connector SM3ZS067U310ABR120 



\section{Chapter 3}

\section{Fabrication Technology}

This chapter details the process sequence used to fabricate the blood typing device. Standard microfabrication processing techniques are used in order to make the process

flow industry compliant and eventually viable. A primary objective was to fabricate a working sensor. The secondary objective was to make sure that the yield was high enough to warrant investment at an industrial level.

\subsection{Early Process Development}

Since the process flow needed to be industry compliant a well defined procedure that is already in use at industry level needed to be applied. Microfabrication techniques 
involving lithography for patterning, sputtering for deposition and dry and wet etches for material removal were considered. Since the design had a critical dimension of $2 \mu \mathrm{m}$, standard soft contact UV lithography was considered. The design of equal lines and spaces was challenging to transfer due to spinning conditions, resist uniformity, variation in intensity of UV lamp on the aligner and the develop process. These are described in further detail in the following sections.

\subsection{Process Flow}

The first phase of fabrication started on glass slides, with four devices on each slide (two in case of the 3Linear design). This involved dark field photomasks and devices at the edge of the slides. Devices were placed at the edge so that a standard M.2 edge connector could be used. This created a variety of problems, the most significant of those being an accumulation of photoresist at the edge of the substrate during spincoating process, called edge beading. Thus, the resist at the periphery of the substrate ends up being thicker than the resist towards the center. This leads to non-uniform

exposure and non-uniform development, resulting in devices with missing/deformed features.

After extensive testing using the devices on glass slides, a decision was made to move to glass wafer substrates to increase the number of devices per substrate, as well as to 
reduce the effects of edge beading. This led to an increase in yield from four devices per run to twenty-six. The rest of this chapter details the fabrication procedure, the gist of which is diagrammatically represented in Figure 3.1. 


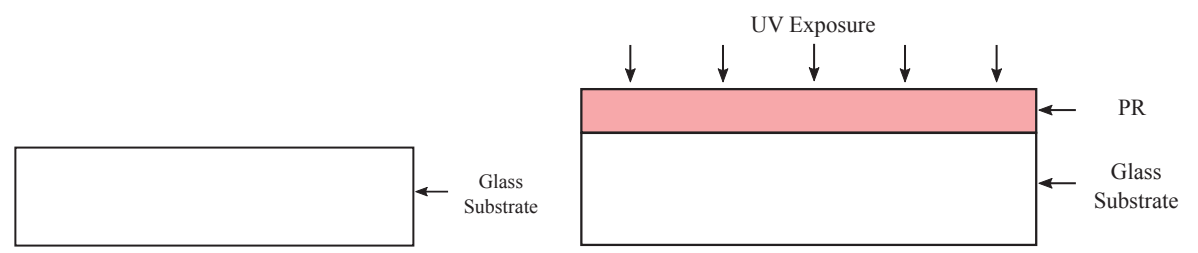

(a) Borofloat33 glass substrate

(b) UV Photolithography

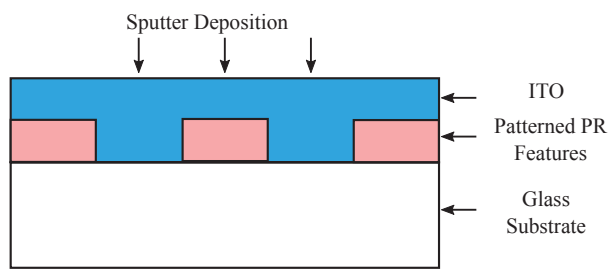

(c) Patterns as seen after development

(d) Sputter deposition of ITO
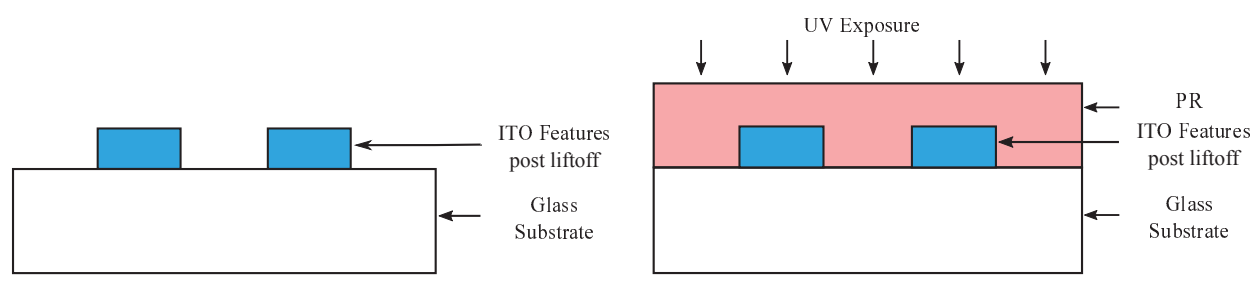

(e) Sample after ultrasonic liftoff

(f) Alignment of metallization layer

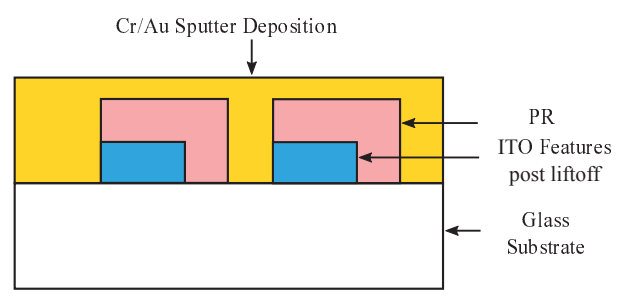

(g) Photoresist after development

(h) $\mathrm{Cr} / \mathrm{Au}$ sputter deposition for metal leads and contacts

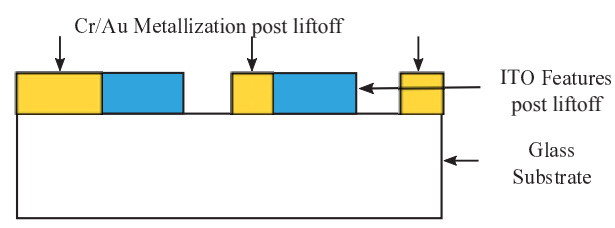

(i) Sample after second ultrasonic liftoff

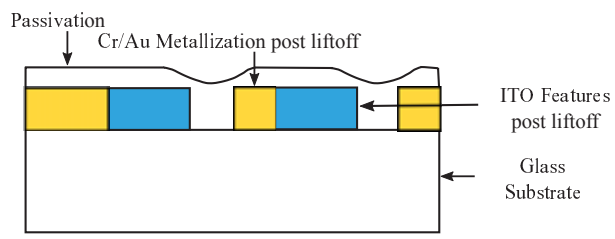

(j) Sample after passivation

Figure 3.1: Fabrication procedure 


\subsection{Choice of substrate}

Three different materials were chosen for their optical properties. Since all the electrical test results were verified optically, there was a need to obtain substrates that are transparent in the visible range of the spectrum. At this stage, the devices were to serve two purposes: optical testing in parallel with the capacitive testing to verify results and validate the method.

\subsubsection{Glass Slides}

Initially the devices were fabricated on standard $3^{\prime \prime} \times 1^{\prime \prime}$ glass slides (obtained from Fisher Scientific). These glass slides are clear, corrosion resistant, with ground edges and are manufactured in a thickness of $1 \mathrm{~mm}$. They have high transmittance in the visible range but suffer from various defects. The edges are usually not cut very precisely. Also, it was observed that when photoresist was spun on them, it tended to accumulate around the edges (edge beading). Thus, the features which are located

close to the edge did not transfer consistently. Therefore, there was a need to look for other substrates that have equally good transparency. 


\subsubsection{Zeonex ${ }^{\circledR}$}

Cyclo Olefin Copolymer substrates, sold under the trade name of Zeonex by Zeon Chemicals have $92 \%$ transparency in the visible range. The material is biocompatible and has a moderately high glass transition temperature $\left(102^{\circ} \mathrm{C}\right.$ to $163^{\circ} \mathrm{C}$ depending on the product grade). Zeonex 790R substrates are available as sheets measuring $150 \mathrm{~mm} \times 150 \mathrm{~mm} \times 2 \mathrm{~mm}$. These substrates were considered for their high transparency (Figure 3.2) and considerably higher glass transition temperature (higher than most plastics). However, it was concluded that this material could not be used for this device in its initial stages, as Zeonex sheets are readily available in a thickness of 2mm, and the edge connectors by TE Connectivity A115901CT-ND and A115905CT-ND are not suitable for substrate thicknesses of over $1.57 \mathrm{~mm}$. Also, it was concluded that all initial devices should be built on a substrate that the entire team was familiar with, in order to ease processing.

\subsubsection{Borofloat $33{ }^{\circledR}$ Borosilicate Glass Wafers}

4" Borofloat 33 glass wafers were obtained from University Wafers in order to elevate the processing to wafer scale and make sure that multiple samples were obtained per fabrication run. Also, these wafers have better uniformity and are pre-examined 


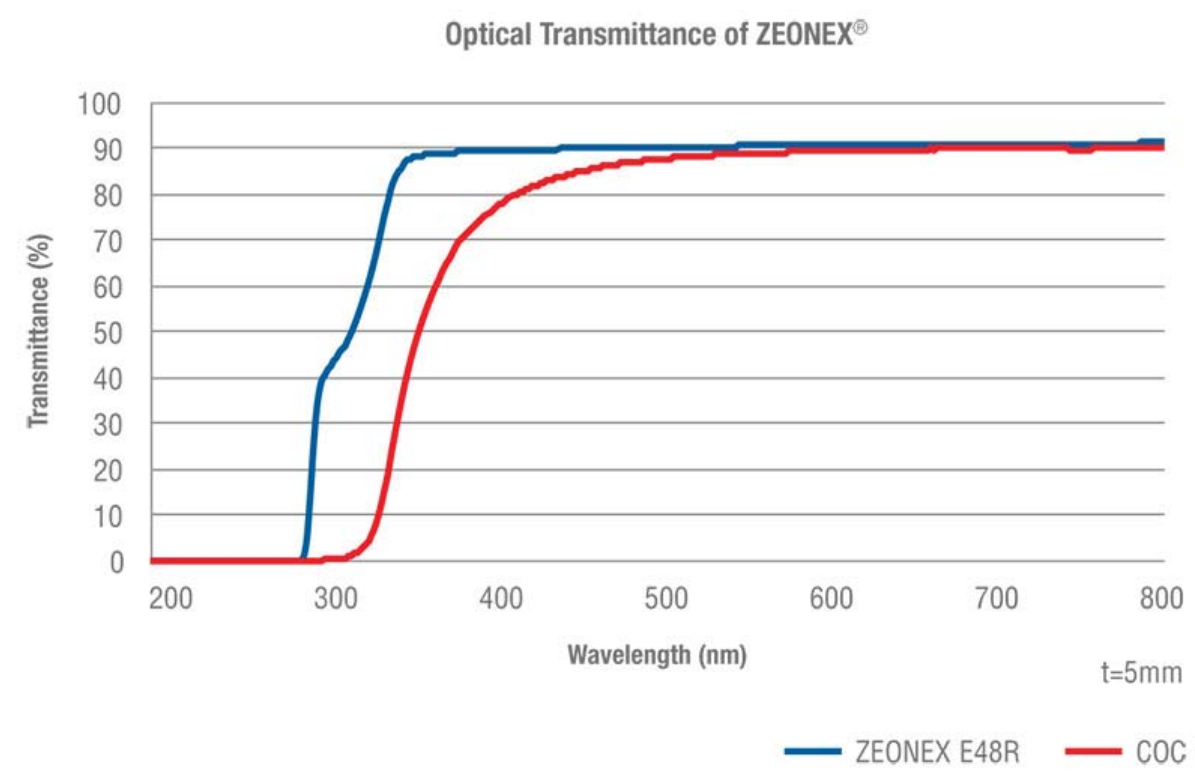

Figure 3.2: Optical transmission of Zeonex in visible and near-visible range $[1]$

for faults. This results in a better quality substrate that is highly transparent and resistant to chemicals and mechanical stresses.

\subsection{Sensing Electrodes}

Sensing electrodes were patterned on glass slides using the process flow described in Table 3.1 and 3.2 .

In order to correctly transfer the pattern from dark field masks, various positive photoresists were explored. At first PR1-2000A, obtained from Futurrex, Inc. was considered. PR1-2000A was spun to a thickness of $2 \mu \mathrm{m}$. However, Futurrex resists 
Table 3.1

Process flow for sensing electrodes using dark field masks and positive photoresist

\begin{tabular}{ll}
\hline \multicolumn{2}{c}{ Sensing electrode layer using Cr/Au } \\
\hline Spin photoresist & Shipley $1827,7000 \mathrm{rpm}$ for $40 \mathrm{~s}$ \\
Prebake & $3 \mathrm{~min}, 120 \mathrm{C}$ \\
Exposure & $160 \mathrm{~mJ} / \mathrm{cm}^{2}$ \\
Develop & $40 \mathrm{~s} \mathrm{in} \mathrm{MF} 319$ \\
Postbake & $3 \mathrm{~min}, 115 \mathrm{C}$ \\
Trion plasma clean & $15 \mathrm{~s}, 98 \mathrm{sccm} \mathrm{O}, 100 \mathrm{~W} \mathrm{ICP,50W} \mathrm{RIE}$ \\
Deposition & $40 \mathrm{~nm} \mathrm{Cr} / 120 \mathrm{~nm} \mathrm{Au}$ \\
Liftoff & $10 \mathrm{~min} \mathrm{sonication} \mathrm{in} \mathrm{RR4}$ \\
\hline
\end{tabular}

Table 3.2

Process flow for sensing electrodes using clear field masks and negative photoresist

\begin{tabular}{ll}
\hline \multicolumn{2}{c}{ Sensing electrode layer using ITO } \\
\hline Spin photoresist & Futurrex NR9-1500P, 5000rpm for $40 \mathrm{~s}$ \\
Prebake & $90 \mathrm{~s}, 120^{\circ} \mathrm{C}$ \\
Exposure & $210 \mathrm{~mJ} / \mathrm{cm}^{2}$ \\
Post exposure bake & $3 \mathrm{~min}, 120^{\circ} \mathrm{C}$ \\
Develop & $6 \mathrm{~s}$ \\
Deposition & $120 \mathrm{~nm}$ ITO \\
Liftoff & $90 \mathrm{~min}$ sonication in RR4 \\
\hline
\end{tabular}

are compatible with Futurrex Developer RD6, which is a very quick TMAH based developer. It took the slides 6 seconds to fully develop, and over and underdevelopment would not yield the features correctly.

Thus, an effort was made to use a developer which has a develop time of around a minute so as to make the processing more controllable. 
This was the motivation to choose Shipley's line of positive photoresists over Futurrex. Shipley 1827 was spun to a thickness of $1.7 \mu \mathrm{m}$, softbaked for 3 minutes at $115^{\circ} \mathrm{C}$ and then exposed on an EV620 Aligner using a dose of $150 \mathrm{~mJ} / \mathrm{cm}^{2}$.

This was developed in Resist Developer MF319 for 40 seconds to resolve the $3 \mu \mathrm{m}$ equal lines and spaces. This process is described in Table 3.1.

This was followed by a sputter deposition process on Perkin-Elmer Randex Sputtering System Model 2400 the 6 " sputter deposition tool.

During phase one of wafer scale processing, a 120nm Au film was used to form the sensing electrodes. A 40nm Cr layer was used to improve adhesion of Au to the glass substrate. A blanket film was deposited, and then lifted off using ultrasonics to obtain the electrodes.

When the fabrication technique was perfected using $\mathrm{Cr} / \mathrm{Au}$ electrodes and measurements taken, Indium Tin Oxide was used to fabricate the sensing electrodes. Indium Tin Oxide (ITO) is a conductive oxide that is transparent [23], and since transparency was a required characteristic to facilitate optical verification of sensing data, i.e., the movement of cells in the presence of an electric field, ITO was the preferred material $[24]$.

The targeted thickness was $120 \mathrm{~nm}$, the same as the thickness of deposited Au. ITO 
was deposited at $100 \mathrm{~W}$ for 13 minutes on a $4^{\prime \prime}$ patterned glass wafer. This was followed by liftoff using ultrasonication to reveal the underlying features. This is shown in Figure 3.2 and the process flow is described in Table 3.2.

It was concluded that to further reduce the sheet resistance of ITO, an annealing step was required [25]. After a $30 \mathrm{~min}$ hotplate anneal at $200^{\circ} \mathrm{C}$, it was observed that the sheet resistance of ITO dropped. This was validated from a characterization step that is detailed in Table 3.3 .

Table 3.3

Sheet resistance for $80 \mathrm{~nm}$ sputter deposited ITO film without annealing and with a hotplate anneal at $200^{\circ} \mathrm{C}$ for 30 mins

\begin{tabular}{lll}
\hline Substrate & \multicolumn{2}{c}{ Sheet resistance } \\
\hline & Without anneal & With anneal \\
\hline Si & $80 \Omega /$ square & $60 \Omega /$ square \\
Glass & $1.2 \Omega /$ square & $200 \Omega /$ square \\
\hline
\end{tabular}

\subsection{Metallization Layer for Electrodes}

The metallization layer was aligned to the sensing electrode layer. This layer contained all the leads from the sensing electrodes leading up to the contact pads. The contact pads were sized $2.5 \mathrm{mmx} 250 \mathrm{~m}$, with a pitch of $500 \mathrm{~m}$, keeping in mind the footprint of the edge connector. The lithography step was exactly the same as that for the sensing electrode layer. This is reiterated in Table 3.4. 
Table 3.4

Process flow for metallization layer using clear field masks and negative photoresist

\begin{tabular}{ll}
\hline Metallization layer & \\
\hline Spin photoresist & Futurrex NR9-1500P, 5000rpm for $40 \mathrm{~s}$ \\
Prebake & $90 \mathrm{~s}, 120^{\circ} \mathrm{C}$ \\
Exposure & $210 \mathrm{~mJ} / \mathrm{cm}^{2}$ \\
Post exposure bake & $3 \mathrm{~min}, 120^{\circ} \mathrm{C}$ \\
Develop & $6 \mathrm{~s}$ \\
Deposition & $25 \mathrm{~nm} \mathrm{Cr} / 120 \mathrm{~nm} \mathrm{Au}$ \\
Liftoff & $90 \mathrm{~min}$ sonication in RR4 \\
\hline
\end{tabular}

$\mathrm{Cr} / \mathrm{Au}$ was used as the lead material. 120nm of Au was deposited on a patterned substrate using $25 \mathrm{~nm}$ of $\mathrm{Cr}$ as an adhesion layer. Again, this was lifted off using ultrasonication to form the leads and contact pads.

It was noticed that the ultrasonic liftoff method was causing damage to the underlying ITO layer. Thus, a change in the process flow was proposed. An etch process for the metallization layer was defined and after the contact pads and leads were etched, the sensing electrode layer was aligned to it.

Gold etchant Type TFA was obtained from Transene Company, Inc. The wafer was dipped in the etchant for 25 seconds after patterning the blanket $\mathrm{Cr} / \mathrm{Au}$ layer to reveal the contact pads and leads. This was rinsed in DI water and then dipped in Chromium Etchant CR7 until the pads and leads were clearly exposed.

This layer was then aligned to the sensing electrode layer and patterned using the 
process described in Section 3.2.

\subsection{Passivation}

In order to isolate the metal leads and sensing electrodes from red blood cells a layer of passivating material was deposited. Parylene-C was the first choice since it is a transparent, biocompatible material [26][27]. A Specialty Coating Systems Parylene Coater was used to deposit 190nm of Parylene-C onto the substrates.

The process was characterized and it was determined that the thinnest uniform coating that could be obtained was $190 \mathrm{~nm}$. This was coated using $0.195 \mathrm{~g}$ of the dimer.

Diced glass slides were then used to mask the device and expose just the pads. This sample was dry etched in an oxygen plasma at 100W for 1 minute to completely remove the passivation.

It was noticed that there were some adhesion issues between the glass substrate and Parylene-C. There were pinholes observed in the Parylene and the passivation layer would peel off. This was attributed to the intrinsic stress of the deposited film [28]. The quality of the film is shown in Figure 3.3

Also, fabrication on a substrate that was already coated with parylene proved difficult, 


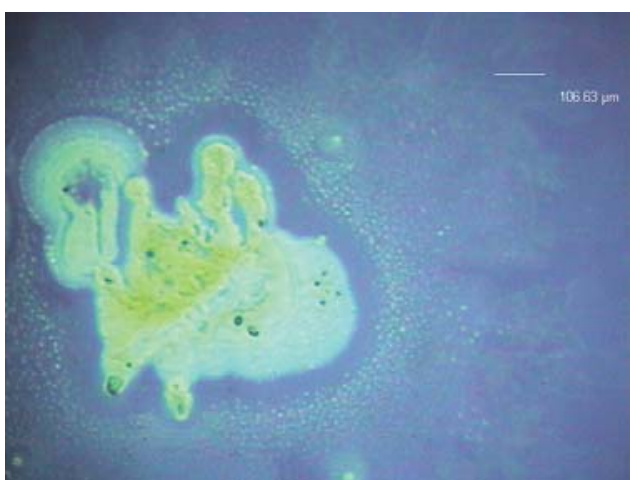

(a) Defects in Parylene-C layer 1

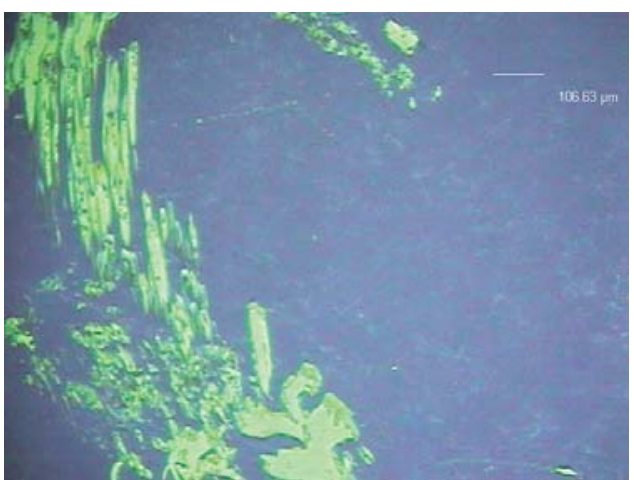

(b) Defects in Parylene-C layer 2

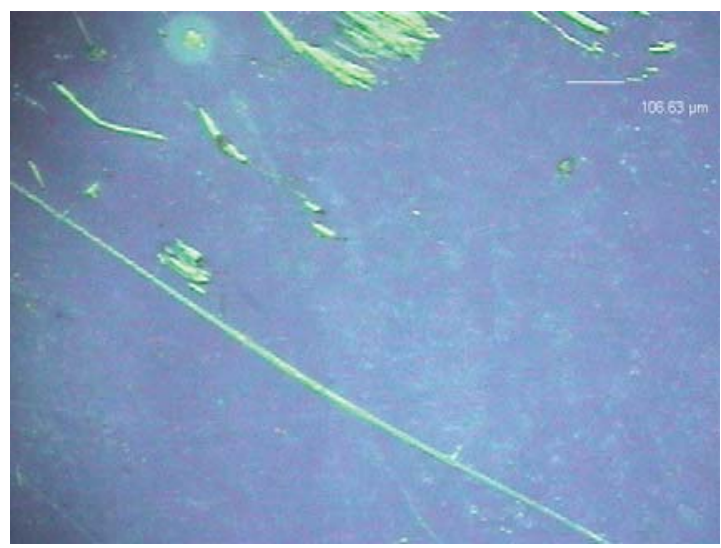

(c) Defects in Parylene-C layer 3

Figure 3.3: Parylene-C film showing defects

since the quality of the film was already compromised. A major difficulty was to get the photoresist to spin in a uniform layer. The liftoff process too damaged the passivation, expanding pinholes and peeling off the layer.

Literature search revealed that adhesion of Parylene-C to a surface improves with more effective mechanical interlocking. The higher the value of surface roughness, better is the mechanical interlocking, leading to improved adhesion [27].

To improve adhesion of the Parylene-C layer to the underlying substrate surface 
treatment using Silane A174 was performed. 3-(Trimethoxysilyl)propyl methacrylate 98\% was obtained from Sigma Aldrich. The Parylene Adhesion promotion treatment process flow is described below [29]:

- $500 \mathrm{~mL}$ of IPA, $500 \mathrm{~mL}$ DI water and $10 \mathrm{~mL}$ Silane A174 were mixed together in a magnetic stirrer for 2.5hours.

- The wafer was immersed in IPA for 5 mins.

- The wafer was then immersed in the IPA/Silane A174/DI water mixture for 30 mins.

- This was allowed to dry upright for 30mins.

- It was immersed in IPA again for 10 mins.

- The wafer was then baked on a hot plate at $110^{\circ} \mathrm{C}$ for $10 \mathrm{mins}$.

- It was then loaded into the SCS Parylene Labcoater for deposition.

LCR test data of sample device B45 is shown in Table 3.5.

The data is inconsistent with the values obtained during all other testing. The dry measurements show values that are an order of magnitude higher than expected. Also, some values of resistance registered as negative. This particular device which 
Table 3.5

LCR test data of B45 showing inconsistencies due to passivation

\begin{tabular}{|c|c|c|c|c|c|c|c|c|}
\hline $\mathrm{B} 45,3$ Linear & $\mathrm{Cp}(\mathrm{pF})$ & $\mathrm{Rp}(\mathrm{M} \Omega)$ & $\mathrm{Cp}(\mathrm{pF})$ & $\mathrm{Rp}(\mathrm{M} \Omega)$ & $\mathrm{Cp}(\mathrm{pF})$ & $\mathrm{Rp}(\mathrm{M} \Omega)$ & $\mathrm{Cp}(\mathrm{pF})$ & $\mathrm{Rp}(\mathrm{M} \Omega)$ \\
\hline $4 \& 5$ & 0.1333 & - & 0.1415 & $95+$ & 0.2279 & 1.031 & 0.6168 & 0.1724 \\
\hline $5 \& 6$ & 0.127 & - & 0.1363 & 78 & 0.1965 & 1.568 & 0.5533 & 0.1994 \\
\hline $6 \& 7$ & 0.1254 & - & 0.1362 & 70 & 0.1736 & 3.41 & 0.5312 & 0.203 \\
\hline $7 \& 8$ & 0.1233 & - & 0.1343 & 72 & 0.1512 & 70 & 0.5108 & 0.2165 \\
\hline $8 \& 9$ & 0.0962 & - & 0.0888 & - & 0.0992 & -24.5 & 0.264 & 0.2569 \\
\hline $9 \& 10$ & 0.1158 & - & 0.1073 & - & 0.1183 & -26.5 & 0.4083 & 0.1784 \\
\hline $10 \& 11$ & 0.1352 & - & 0.1472 & $95+$ & 0.1549 & -37 & 0.6362 & 0.2095 \\
\hline
\end{tabular}

was passivated with Parylene yielded unstable results on the LCR meter. At first, it was hypothesized that the probes could be corroded. Fresh probes were thus obtained from The Micromanipulator Company and testing of B45 using these revealed the same issue. This inconsistency was attributed to defects in the Parylene layer based on evidence from data, i.e., inconsistent results as well as from microscope images shown.

It was concluded that there is a need to find a material that is transparent, can be deposited easily and would form a good passivation layer. For this, high-k dielectrics were studied and it was concluded that Hafnium Dioxide (hafnia) would be a good choice. It was readily available as a target in the Perkin-Elmer Randex Sputtering System Model 24008 inch. The relative permittivity of this material was found to be 25, and as compared to 3 in case of Parylene, it was concluded that $1 / 8$ the thickness of Parylene was desired to make sure that the value of capacitance would be similar. This is demonstrated by Equation 3.1. 


$$
\begin{aligned}
C & =\epsilon \frac{A}{D} \\
\epsilon_{\mathrm{PaC}} & =3 \\
\epsilon_{\mathrm{HfO} 2} & =25 \\
\frac{C_{P a C}}{C_{\mathrm{HfO}_{2}}} & =\frac{\epsilon_{\mathrm{PaC}} \frac{A}{\overline{D P a C}_{P f O_{2}} \frac{A}{D_{H f O_{2}}}}}{\frac{C_{\mathrm{PaC}}}{C_{\mathrm{HfO}_{2}}}}=\frac{\frac{3}{D_{P a C}}}{\frac{25}{D_{\mathrm{HfO}}}} \\
\frac{C_{\mathrm{PaC}_{2}}}{C_{\mathrm{HfO}_{2}}} & =\frac{3}{25}
\end{aligned}
$$

The etch process that had been defined in case of the parylene coating could not be used here since the only etchant for hafnia is hydrofluoric acid, which would attack the glass substrate. Thus, there was a need to mask the contact pads to prevent any accidental deposition.

The deposition rate was first characterized using $\mathrm{Si}$ and glass samples that were then measured ellipsometrically to form a process that would be repeatable. It was concluded that hafnia, when deposited at $700 \mathrm{~W}$ has a rate of $1 \mathrm{~nm} / \mathrm{min}$. Thus a 25 minute run was defined and substrates with the contact pads were masked using high temperature Kapton tape. A blanket coating of hafnia was deposited. The samples were unloaded and the tape was stripped. 
On the first few samples it was noticed that the tape left some residue. This was attributed to the mechanical force acting on the substrate during the sputtering run. An effort was made remove the adhesive using a variety of solvents. When standard solvents such as acetone, IPA, ethanol and MIBK did not work, the substrate was dipped in boiling acetone and scrubbed using a swab. The tape residue was still there. Straight chain polymers like hexane were tried, but did not yield positive results. The substrate was then dipped in cyclic polymers such as toluene. This too did not yield positive results.

An oxygen plasma ash was attempted next but that carbonized the tape residue to yield a black, amorphized surface that was no longer usable. A 6" Si wafer was then diced into $3 \mathrm{~mm}$ strips and secured to the substrate using Kapton tape to cover the contact pads. Appropriate care was taken to ensure that the tape was used at the bottom face of the substrate so as to eliminate the need to clean the surface to spin photoresist for the drive electrodes. A uniform coating of hafnia was obtained and was measured to be between 25 and $30 \mathrm{~nm}$ for a variety of samples. This is shown in Figure 3.4.

With hafnia as passivation, device B45 was tested again, and data obtained is shown in Table 3.6. These measurements were stable and the dry measurements in particular were consistent with the expected results.

This is compared in Figure 3.5. This further corroborated the decision to use hafnia 


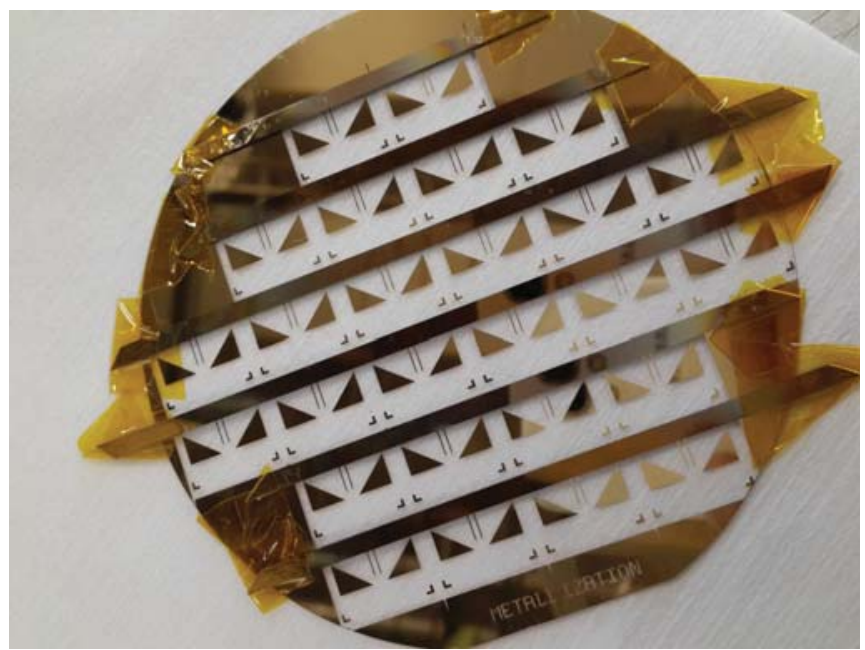

Figure 3.4: Masked wafer for $\mathrm{HfO}_{2}$ deposition

Table 3.6

LCR test data for hafnia coated device B45 after Parylene-C was stripped

\begin{tabular}{|c|c|c|c|c|c|c|c|c|}
\hline & \multicolumn{2}{|c|}{ DRY } & \multicolumn{2}{c|}{ E-pure } & \multicolumn{2}{c|}{$\mathrm{PBS}$} & \multicolumn{2}{c|}{$\mathrm{RBC}$} \\
\hline $\mathrm{B} 45,3$ Linear & $\mathrm{Cp}(\mathrm{pF})$ & $\mathrm{Rp}(\mathrm{M} \Omega)$ & $\mathrm{Cp}(\mathrm{pF})$ & $\mathrm{Rp}(\mathrm{M} \Omega)$ & $\mathrm{Cp}(\mathrm{pF})$ & $\mathrm{Rp}(\mathrm{M} \Omega)$ & $\mathrm{Cp}(\mathrm{pF})$ & $\mathrm{Rp}(\mathrm{M} \Omega)$ \\
\hline $4 \& 5$ & 0.0641 & & 0.1011 & & 0.1083 & & 0.0798 & \\
\hline $5 \& 6$ & 0.0605 & & 0.1032 & & 0.1122 & 70 & 0.0829 & $97+$ \\
\hline $6 \& 7$ & 0.0721 & & 0.1112 & & 0.1193 & & 0.0895 & \\
\hline $7 \& 8$ & 0.0502 & & 0.0875 & & 0.0978 & & 0.0632 & \\
\hline $8 \& 9$ & 0.0537 & & 0.095 & & 0.1042 & & 0.0704 & \\
\hline $9 \& 10$ & 0.0599 & 20.5 & 0.0998 & & 0.1094 & & 0.0748 & \\
\hline $10 \& 11$ & 0.0485 & & 0.0873 & & 0.0964 & & 0.0624 & \\
\hline
\end{tabular}

as the dielectric and not Parylene-C.

The trend is particularly visible in case of dry measurements taken on B45 with Parylene-C as dielectric. The value is much higher than the expected value calculated through analytical model and the simulation. When the same device was stripped of $\mathrm{Pa}-\mathrm{C}$ and coated with hafnia, the values obtained adhered to the expected values. This inconsistency in the $\mathrm{Pa}-\mathrm{C}$ measurement was attributed to pinhole defects in $\mathrm{Pa}-\mathrm{C}$ and its lack of mechanical strength under stress. 


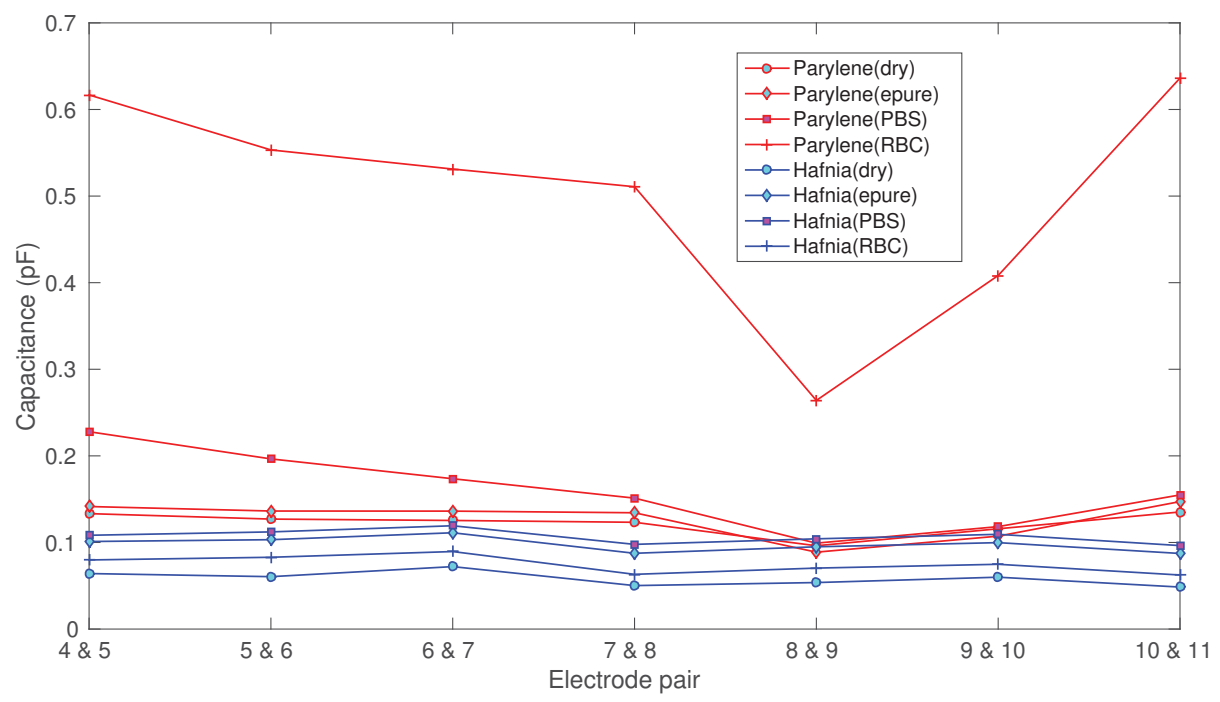

Figure 3.5: Comparison of LCR test data between Parylene coated and hafnia coated device. This data is for the same device B45 which was tested twice, with two different dielectrics.

These samples were processed further with hafnia to pattern the drive layer.

\subsection{Drive Electrodes}

Drive electrodes were patterned on the substrates using the process flow described in Table 3.7. Clear field masks were acquired from HTA Photomask and negative resist, Futurrex NR9-1500P was spun at $5000 \mathrm{rpm}$ for 40s. This was followed by a hot plate prebake at $120^{\circ} \mathrm{C}$ for $90 \mathrm{~s}$. This wafer was aligned to the underlying layers and exposed at $210 \mathrm{~mJ}$. A post exposure bake at $120^{\circ} \mathrm{C}$ for 3 mins revealed the latent image which was examined under the microscope in the clean room. The polarizer on the microscope was utilized to view a pseudo-3D image. This sample was then 
developed in RD6 for 6s.

Table 3.7

Process flow for drive electrodes using clear field masks and negative photoresist

\begin{tabular}{ll}
\hline \multicolumn{2}{c}{ Sensing electrode layer using ITO } \\
\hline Spin photoresist & Futurrex NR9-1500P, 5000rpm for $40 \mathrm{~s}$ \\
Prebake & $90 \mathrm{~s}, 120^{\circ} \mathrm{C}$ \\
Exposure & $210 \mathrm{~mJ} / \mathrm{cm}^{2}$ \\
Post exposure bake & $3 \mathrm{~min}, 120^{\circ} \mathrm{C}$ \\
Develop & $6 \mathrm{~s}$ \\
Deposition & $120 \mathrm{~nm}$ ITO \\
Liftoff & $90 \mathrm{~min}$ sonication in RR4 \\
\hline
\end{tabular}

120nm of Au was deposited with a 25nm Cr adhesion layer. This sample was then

lifted off in Futurrex RR4, the resist remover compatible with positive and negative Futurrex resist. In order to protect the underlying layers from the ultrasonic treatment a high frequency with a very low power output was desired. It was observed that this frequency and power setting caused less damage to the underlying $3 \mu \mathrm{m}$ ITO and $\mathrm{Au}$ features at the expense of increasing liftoff time from 10 mins to 90 mins.

\subsection{Singulation}

The wafer was diced using a diamond blade dicing saw with a blade thickness of 10mils to singulate the die. The parameters used for the dicing process are listed in Table 3.8. This die measured $16 \mathrm{~mm} \times 13 \mathrm{~mm}$ and was cut to fit the edge connector. 
Table 3.8

Dicing saw parameters

\begin{tabular}{|c|c|}
\hline PROGRAM ID: & 0 \\
\hline MODE: & 10 \\
\hline DIMENSION 1: & 4173.23 MILS \\
\hline DIMENSION 2: & 4173.23 MILS \\
\hline INDEX 1: & 519.685 MILS \\
\hline INDEX 2: & 637.795 MILS \\
\hline HEIGHT: & 2.000 MILS \\
\hline THICKNESS: & 24.654 MILS \\
\hline ANGLE: & 90.000 DEGREES \\
\hline CUTTING SPEED: & 100.000 MILS/SEC \\
\hline CUT INCREMENT: & 13.000 MILS \\
\hline SPINDLE SPEED: & 30000.0 RPM \\
\hline STOP COUNT: & 999 \\
\hline CUT COUNT: & 1119 \\
\hline
\end{tabular}

\subsection{Fluidic Chamber}

Once the microdevice was ready there was a need to align a PDMS microfluidic chamber to the sensing region. It was critical that this PDMS chamber be centered on the sensing electrodes and the tips of the drive electrodes. The sensing electrodes were placed in such a way that a tolerance of $\pm 2 \mu \mathrm{m}$ was allowed at the tips of the drive electrodes.

A Si wafer was pattered with the fluidic chamber using SU8 2025 negative photoresist using the process flow described in Table 3.9.

PDMS was poured on this pattered Si wafer to create a casting of the chamber. This 
Table 3.9

Process flow for fluidic chamber using SU8 2025 resist

\begin{tabular}{lc}
\hline & Sensing electrode layer using ITO \\
\hline Spin photoresist & MicroChem SU8 $2025,750 \mathrm{rpm}$ for $30 \mathrm{~s}, 1500 \mathrm{rpm}$ for $45 \mathrm{~s}$ \\
Prebake & $1 \mathrm{~min}, 65^{\circ} \mathrm{C}$ and $5 \mathrm{mins}, 95^{\circ} \mathrm{C}$ \\
Exposure & $500 \mathrm{~mJ} / \mathrm{cm}^{2}$ \\
Post exposure bake & $1 \mathrm{~min}, 65^{\circ} \mathrm{C}$ and $5 \mathrm{mins}, 95^{\circ} \mathrm{C}$ \\
Develop & $\mathrm{SU} 8$ developer, 10 mins \\
\hline
\end{tabular}

casting was then cut and aligned to the microdevice using alignment marks described in Chapter 2. A biopsy punch was used to form holes for inlet and outlet ports.

\subsubsection{Well and Chamber}

In order to simplify processing initial testing was done using a well instead of a chamber. The only difference was that the well did not have inlet and outlet ports and was open at the top. This was tested using deionized and biologically pure water (epure water), PBS of different conductivities and RBCs. It was observed that LCR measurements were unstable due to constant movement of fluid in the well. A cover slip was used to cover the well to eliminate the impact of air. However, this too did not yield stable data. A decision was made to use a closed chamber instead.

The initial design of the chamber as described in Section 2.3 involved channels that were at an angle less than $90^{\circ}$ to each other. This resulted in the fluid flowing directly to the outlet bypassing the chamber. Thus a change in the design of the chamber 
was proposed and the new design incorporated channels that were $180^{\circ}$ to each other. The ports were placed in the blank space to the right of the drive electrodes. This device yielded stable measureable data that is documented in Chapter 4 .

\subsubsection{PDMS Bonding}

A variety of methods to bond the PDMS chamber to the microdevice were explored. These included atmospheric plasma, ozone treatment and bonding using tape. Tape was eliminated as a bonding methodology due to leakages in various spots on the device. It was also discovered that PDMS did not bond strongly to parylene coated substrates. This bonding issue accentuated on the need to use hafnia instead of parylene to passivate the electrodes. From previous testing it is known that PDMS bonds very well to hafnia coated glass. Atmospheric plasma and ozone treatment resulted in a permanent PDMS to glass bond and this bonded device is shown in Figure 3.6.

Using the methodology described above, a biosensor was fabricated first on slide scale and then on wafer scale for production on a intermediate scale. Twenty-six devices incorporating 6 different designs of sensing electrodes were patterned on each wafer in order to elevate the processing scale. 


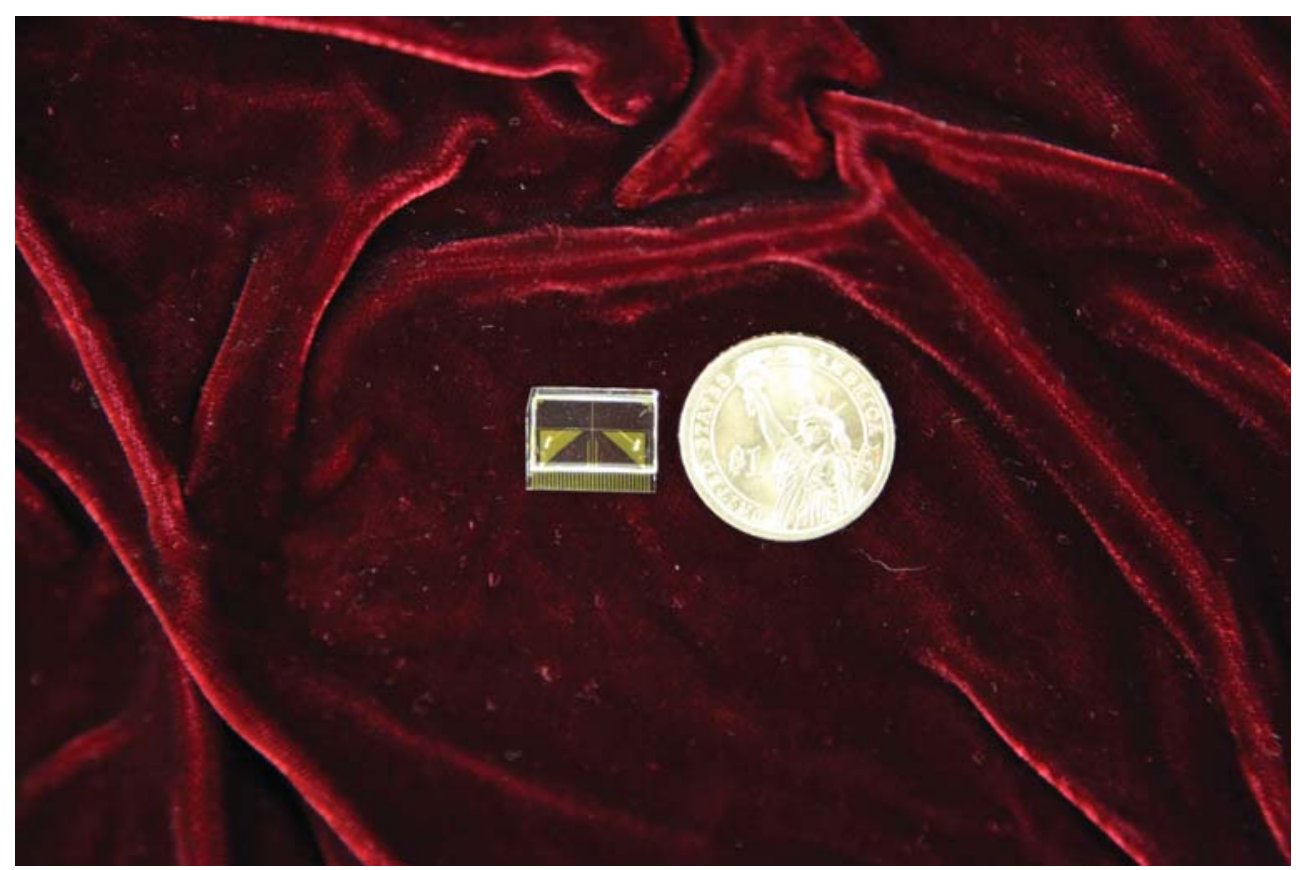

Figure 3.6: Final fabricated device sized to a $\$ 1$ coin for comparison 


\section{Chapter 4}

\section{Testing and Results}

This chapter details the testing procedure that was used for proof of concept analysis. After the chamber was bonded to the microdevice, it was tested using a variety of different solutions. Dry measurements were made to ascertain the effect of passivation, as well as to test for a base capacitance value for the device. Wet measurements were undertaken using PBS of different conductivities, since PBS was the preferred medium for suspending RBCs. Wet measurements using deionized and biologically pure water (epure water) were made also to see effect of an isotonic solution (by comparing this to PBS), as well as to verify the value of measured resistance against a known value. 


\subsection{Instrumentation}

A probe station attached to an HP4284 Precision LCR meter was used to measure values of capacitance and resistance in parallel ( $\mathrm{Cp}$ and $\mathrm{Rp})$. The parallel measurement system was chosen in order to get a higher value of equivalent capacitance so that it could be easily measured and isolated from the noise using on-board electronics on the final device.

The probe station was connected to micromanipulators that had three translational degrees of freedom. The sample was set up in the probe station and was connected to the LCR meter using coaxial cables.

After the sample had been set up, all lead capacitances were compensated for in the instrumentation using the open and closed corrections setting. The capacitance of the coaxial cable and the micromanipulator was separately measured using a different LCR meter and was found to be $104.28 \mathrm{pF}$, making the open and close corrections absolutely necessary for measurements. The targeted capacitance to be measured was in the range of $0.001-20 \mathrm{pF}$, and without zeroing, the actual measurement would be well below the noise floor.

These capacitive results are detailed in Section 4.3. 


\subsection{Treatment of RBCs with Glutaraldehyde}

There was a need to test the devices using red blood cells. However, the Microfabrication Facility at Michigan Technological University is not a biohazard-capable facility and hence a method to deactivate the live tissue was sought. Gordon et al. [30] describe a method to disable the live tissue while still maintaining the structure of the RBC. This was essential to retain the dielectrophoretic response, which is dependent on the polarization of RBCs. This, in turn, is affected by the structure of the cell. Thus, the cells were treated as described and were then suspended in an isotonic solution that was used for testing.

It was noticed that the "pickled" RBCs tend to adhere to the PDMS chamber more than live cells and hence, retesting the same device was challenging without using surfactants to clean the residue from previous testing.

\subsection{Capacitive Testing}

Devices fabricated on glass slides showed high day to day variability. As examples testing data from devices 3Circular and 7Linear are shown. Figure 4.1 shows measurements taken on the 3Circular device on two different days. These measurements 
were taken with and without polystyrene beads (PS) in PBS. These devices were passivated with Parylene-C.

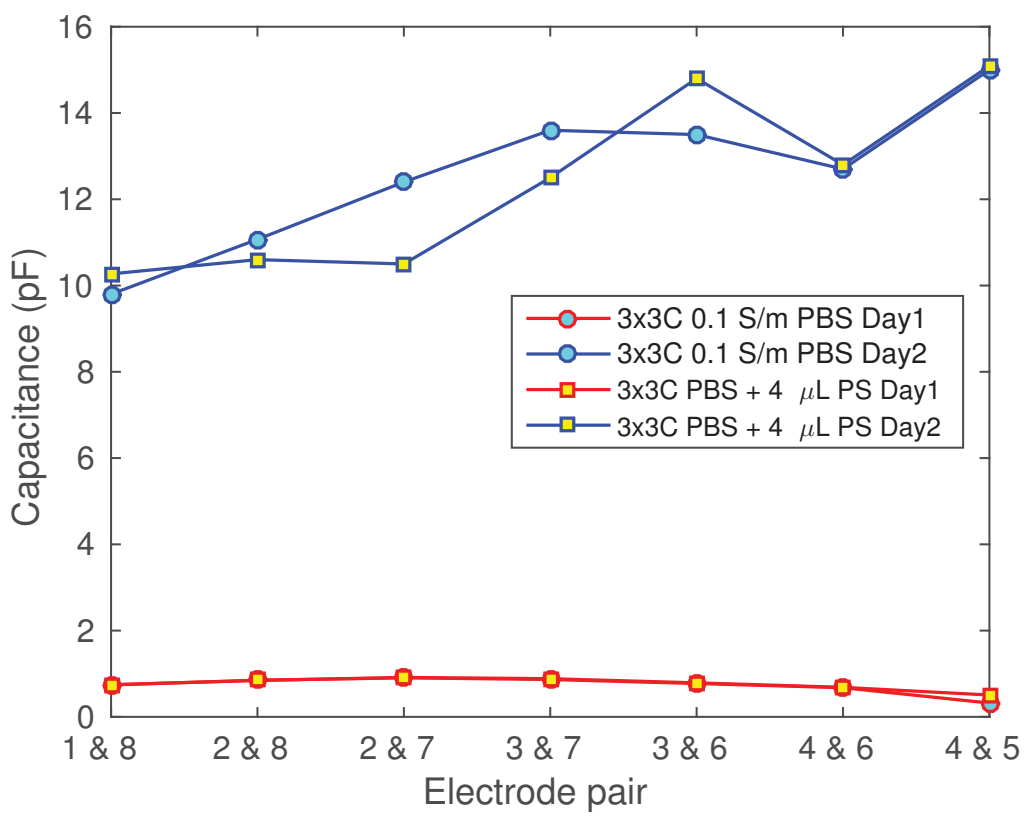

Figure 4.1: Day to day variability in measured values of capacitance in 3 Circular device showing high capacitance on Day 2. This was attributed to the integrity of the Parylene layer being compromised after initial testing. This measurement was done in collaboration with Dr. Hector Moncada.

The capacitance measured on Day 2 was high as compared to the initial measurements. Similar results are seen in case of the 7Linear device (Figure 4.2). This day to day variability was attributed to leakages in the device due to loose sealing of PDMS to Pa-C.

The microfluidic chamber was not properly attached to the substrate and had to be resealed. Also, the integrity of the passivation was questionable because of pinhole defects in the layer. It was theorized that this variability was due to the analyte 


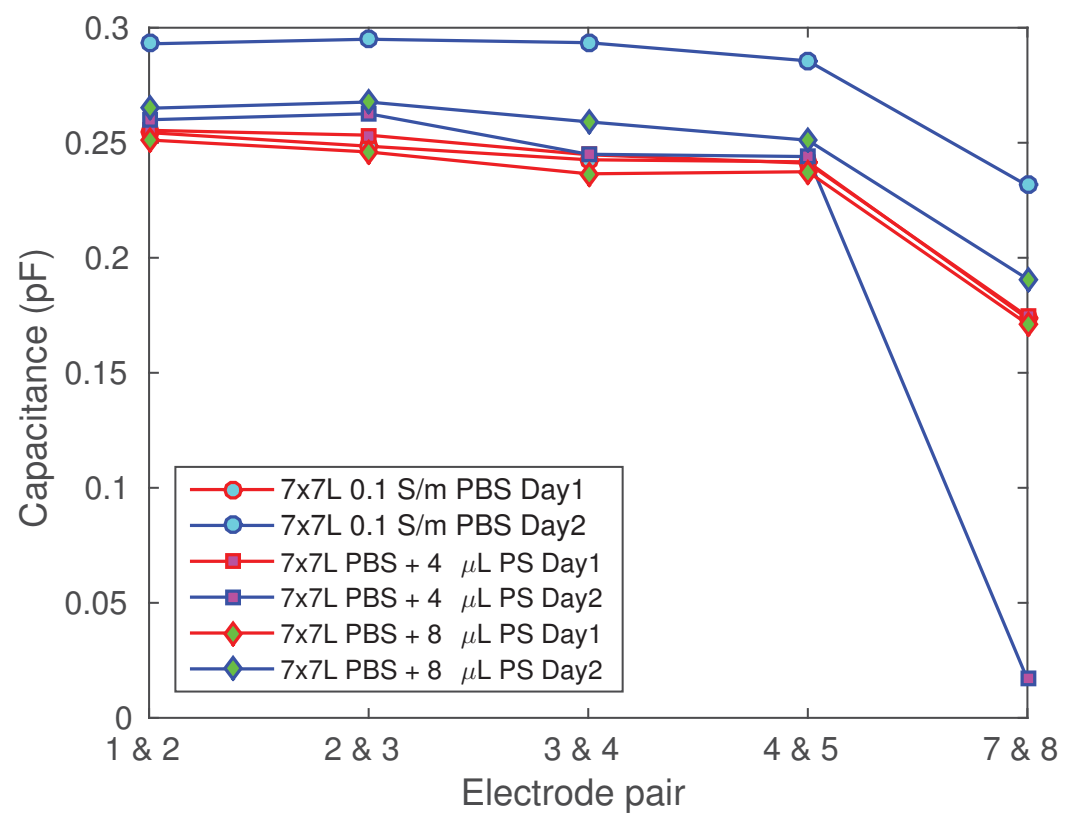

Figure 4.2: Day to day variability in measured values of capacitance in 7Linear device. This was attributed to the integrity of the Parylene layer being compromised after initial testing. This measurement was done in collaboration with Dr. Hector Moncada.

coming in contact with the electrode itself because of poor passivation. Also, there was still some flow in the chamber that could not be controlled due to the leak.

The capacitance test data of device 7Circular is shown in Figure 4.3. While there is a discernible difference between measurements taken with and without PS beads for some electrode pairs, the behavior is not consistent.

The difference is much more apparent in the resistance measurement (Figure 4.4). Testing with PBS and PS beads showed a measureable change in resistance.

This device too was passivated with $\mathrm{Pa}-\mathrm{C}$. The data did prove that it was possible to 


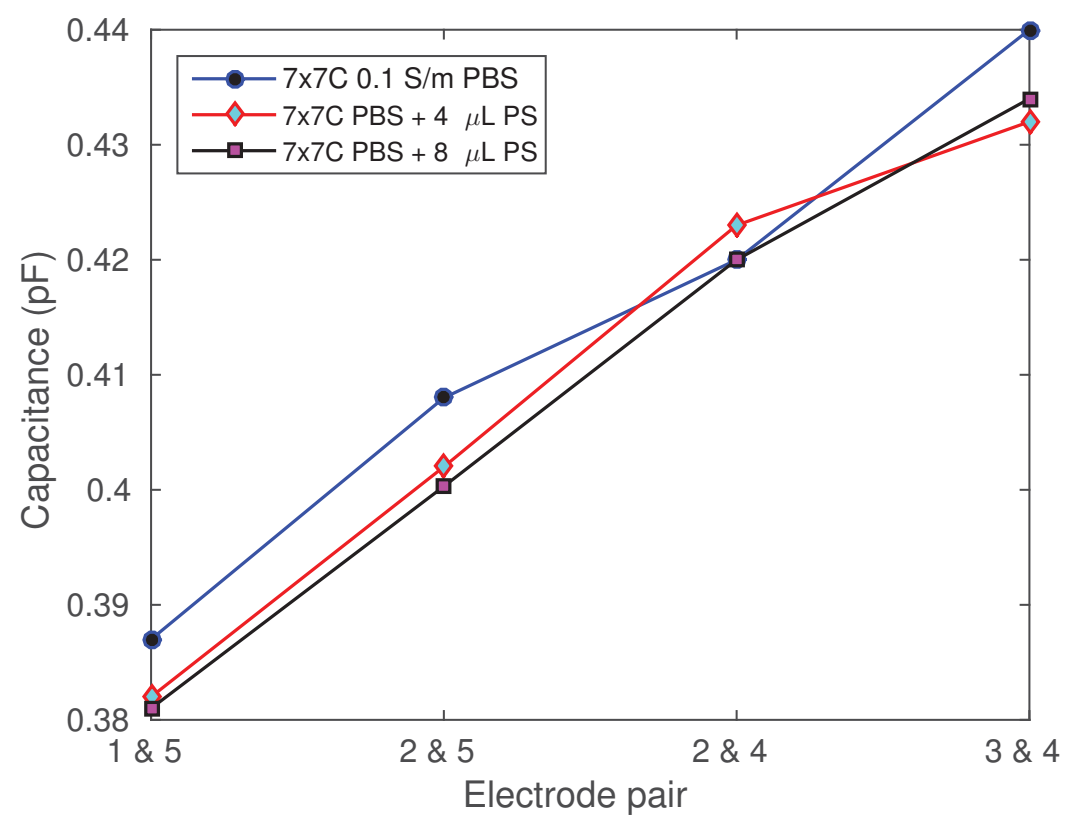

Figure 4.3: Difference in capacitance with and without PS beads for 7Circular device showing inconsistent results, attributed to non-uniformity of passivation. This measurement was done in collaboration with Dr. Hector Moncada.

detect a change but the response was not strong enough. It was thus concluded that in order to minimize the variability provided by $\mathrm{Pa}-\mathrm{C}$ passivation, hafnia should be used as the dielectric.

The 3Circular device shown in Figure 4.5 shows clear differences between capacitance values when different analytes were used. The low capacitance provided by the dry measurement fits in with the modeling results. There is a change between capacitance of PBS and PBS with PS beads and it can be seen for a few electrode pairs.

The resistance of the same 3Circular device (Figure 4.6) showed a clear difference between the readings with epure water and PBS as analytes. 


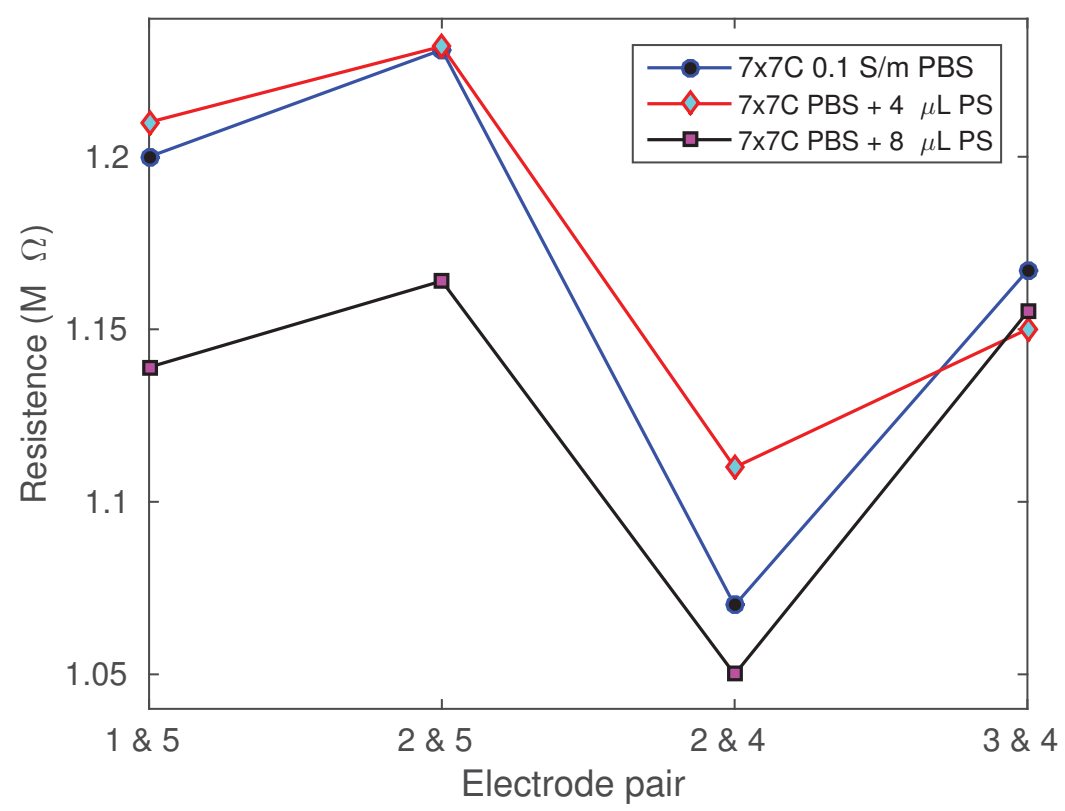

Figure 4.4: Difference in resistance with and without PS beads for 7Circular device showing a clear change in measured values with the analyte was changed. This measurement was done in collaboration with Dr. Hector Moncada.

The 7Linear device too showed a clear variation in capacitance (Figure 4.7) when tested with different analytes. The dry measurement adhered well to the modeling results. There was a clear distinction between results for PBS and PBS with high concentration of PS beads. This helped prove the concept that particles can be detected in solution using a differential measurement.

The resistance data of the same device was collected (Figure 4.8) and this showed some abnormalities. This was attributed to the fact that the driving current was alternating and the dielectric was lossy. The difference in resistance did not take into account all the factors responsible for distinguishing between PBS only and PBS with PS results. 


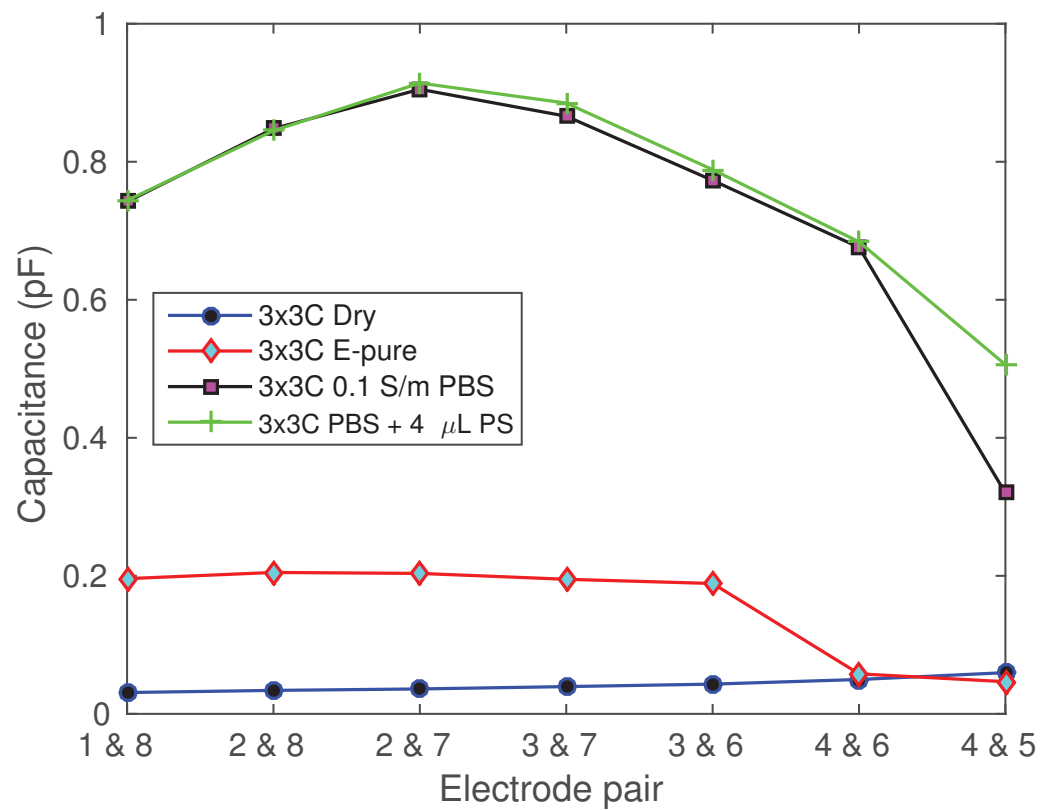

Figure 4.5: Difference in capacitance with and without PS beads for 3Circular device showing discernible difference between values obtained for different analytes. This measurement was done in collaboration with Dr. Hector Moncada.

Test data from a 3Circular device is shown as an example to further corroborate this result. The capacitance of the device (Figure 4.9) with PBS and PBS+PS as analytes showed a discernible change, the resistance results (Figure 4.10) looked very similar to those obtained for the other 3Circular device as shown in Figure 4.6. This proved that passivation with hafnia yielded more repeatable results as compared to $\mathrm{Pa}-\mathrm{C}$.

The 3Linear device showed a clear pattern of change of capacitance (Figure 4.11). However, the graph for resistance (Figure 4.12) had various points of discontinuity. This was due to unstable values obtained because of bad contact between probe needles and contact pads. 


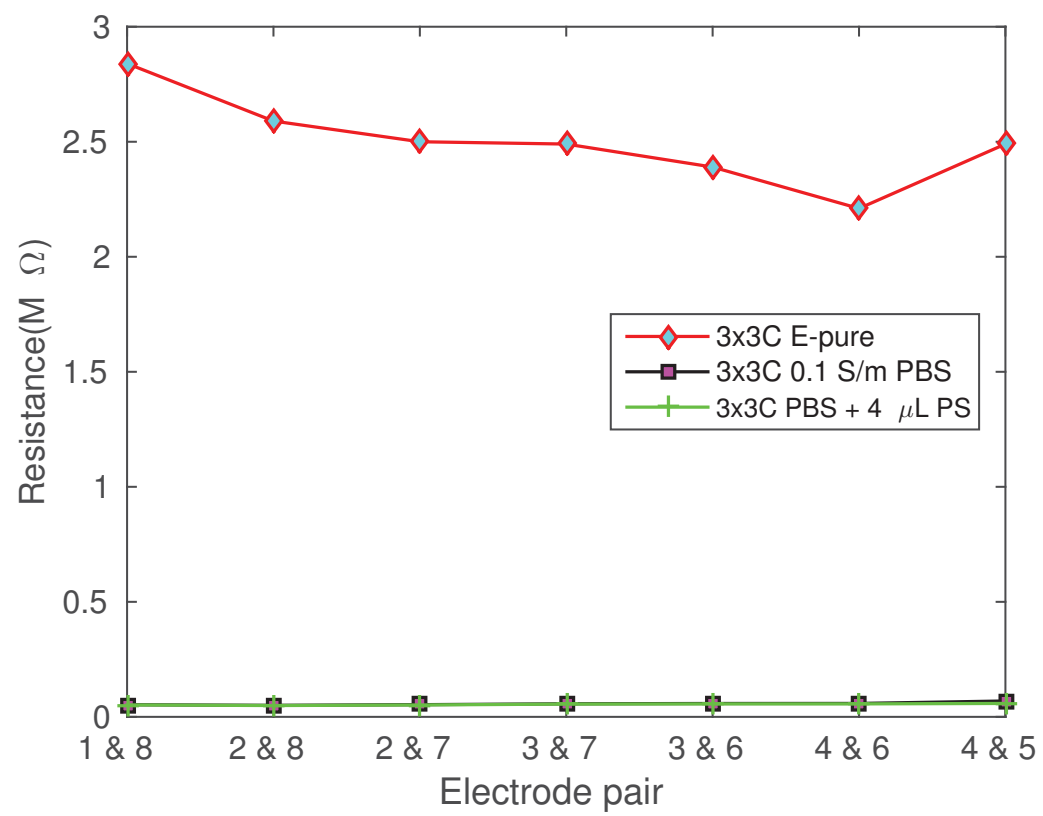

Figure 4.6: Difference in resistance with and without PS beads for 3Circular device showing discernible difference between values obtained for different analytes. This measurement was done in collaboration with Dr. Hector Moncada.

Thus, to see how much of an impact this had over all the data gained thus far, an analysis of mean values and standard deviations was done. As an example data from devices 3Linear and 3Circular is listed out in Tables 4.1 and 4.2 respectively. The value of standard deviation is case of capacitance measurements is low enough for the data to be acceptable.

To further investigate the effect of bad contact between the probe tips and the contact pads, new probes needles were obtained from The Micromanipulator Company and devices were tested. This was done with wafer scale fabricated device D33 that had a $25 \mathrm{~nm}$ hafnia film as passivation. It was seen that the kind of probe tips used had an impact, albeit small, on the measured values. The difference between data obtained 


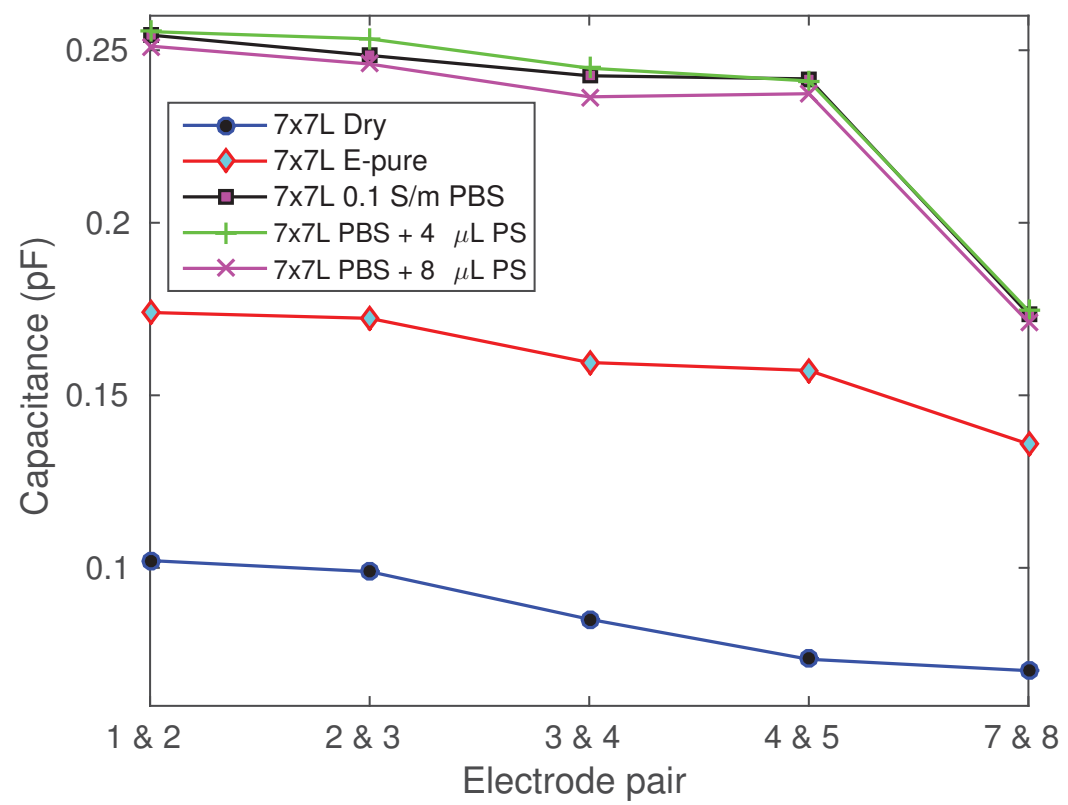

Figure 4.7: Difference in capacitance with and without PS beads for 7Linear device showing discernible difference between values obtained for different analytes. This measurement was done in collaboration with Dr. Hector Moncada.

from two different probe tips is shown in Figure 4.13.

It was seen that the $7 \mathrm{x}$ needle registered higher levels of capacitance as compared to 7x short. The $7 \mathrm{x}$ needle has a tip that is longer and thinner, and it was concluded that $7 \mathrm{x}$ has much higher sensitivity.

This probe tip was chosen for future testing with wafer scale devices. Data was gathered for a hafnia passivated 3Linear device and the difference between capacitances of PBS only and PBS with RBCs was calculated. This difference was computed for different solution conductivities and different conditions of frequency and potential. These results are show in Figure 4.14. 


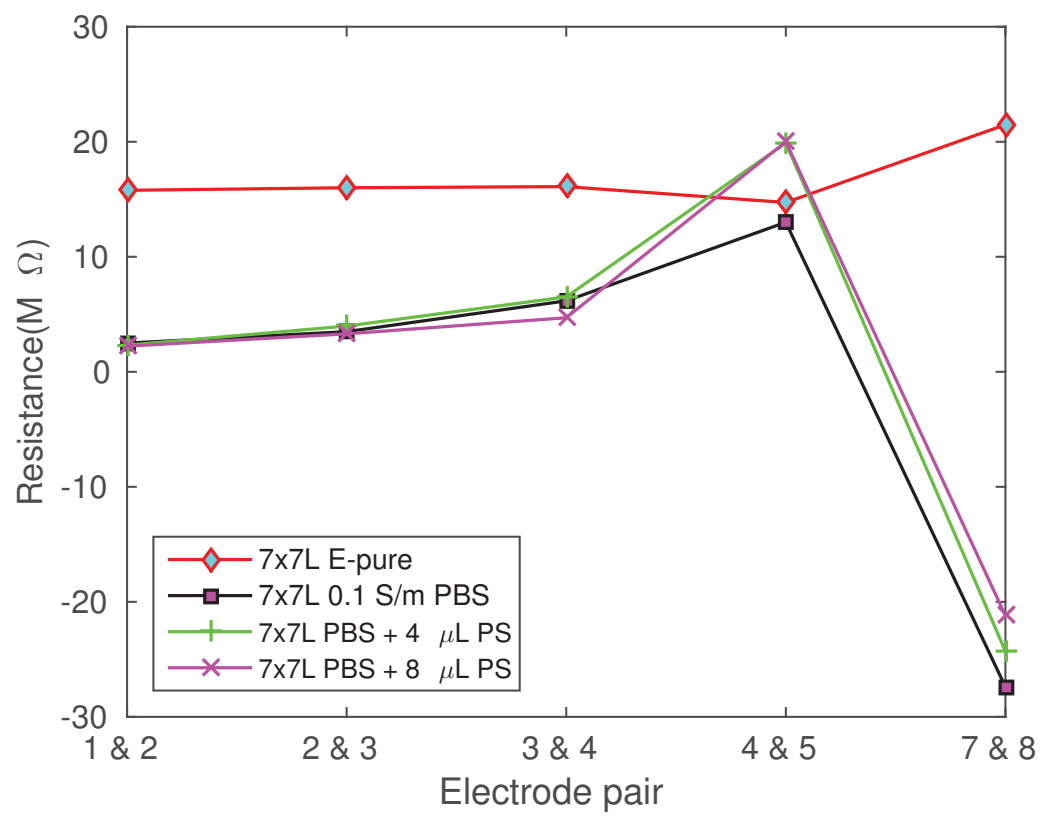

Figure 4.8: Difference in resistance with and without PS beads for 7Linear device showing inconsistent data. This was atrributed to a pure resistance measurement not taking into account all factor at play, leading to a need to gather data on phase angle and impedance. This measurement was done in collaboration with Dr. Hector Moncada.

It was noticed that the sensitivity was highest at a frequency of $1 k H z$. However, the data obtained at this frequency was unstable and the next best case had to be selected.

Figure 4.15 shows highest sensitivity for data collected at $32 \mathrm{kHz}$ and $1 \mathrm{~V}$ for a solution of conductivity $1 \mathrm{~S} / \mathrm{m}$. This was closest to the ideal case since the alpha prototype used a chip that sensed at $32 \mathrm{kHz}$. Also the conductivity of the solution here was $0.1 \mathrm{~S} / \mathrm{m}$, which has been optically shown to provide the best response to a DEP field.

It was noticed that there were some discrepencies in the data obtained during a 


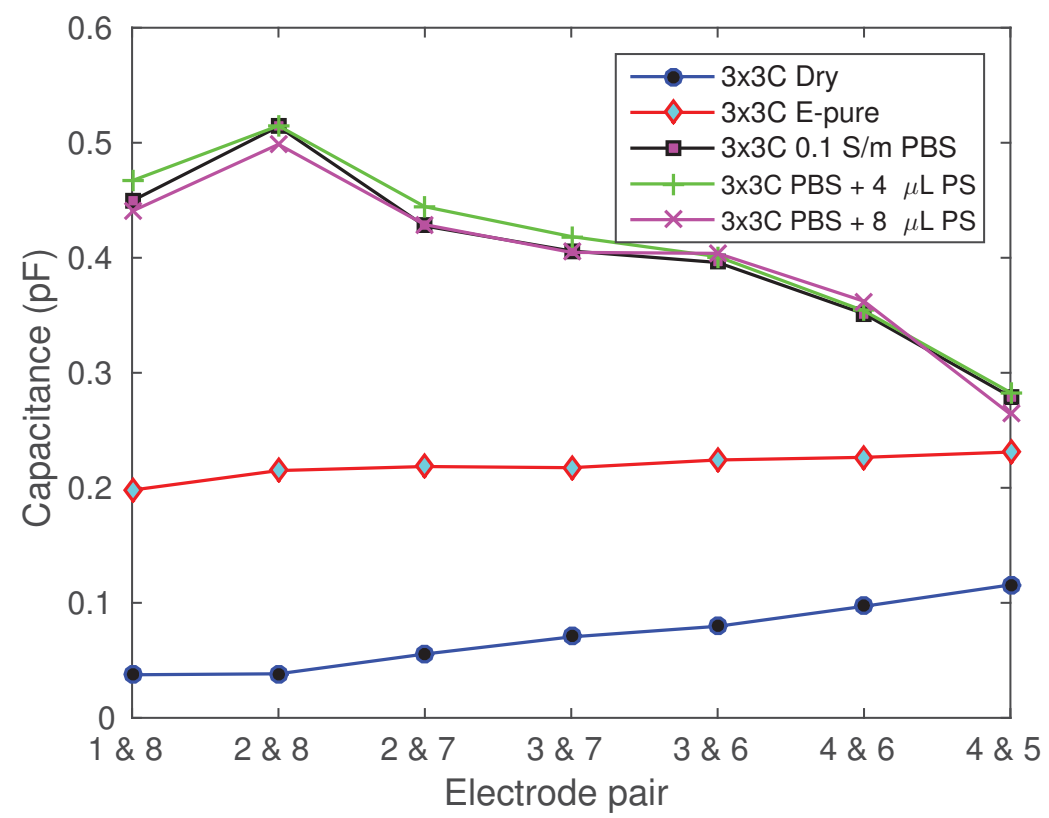

Figure 4.9: Difference in capacitance with and without PS beads for 3Circular device showing discernible difference in values for different analytes. This measurement was done in collaboration with Dr. Hector Moncada.

$\mathrm{Cp} / \mathrm{Rp}$ measurement. The system registered some values of capacitance as negative.

To further investigate this, it was proposed that an impedance measurement along with a phase angle should be done. This would also take into account the variation in inductance, thus providing more information about the behaviour of the device. The impedance data is shown in Table 4.3.

The data adhered well to the general pattern and a change in the capacitance measurement with and without RBCs was seen.

Due the the negative values of capacitance recorded in some of the readings, information about the phase angle was sought to determine if there was an inductive element 


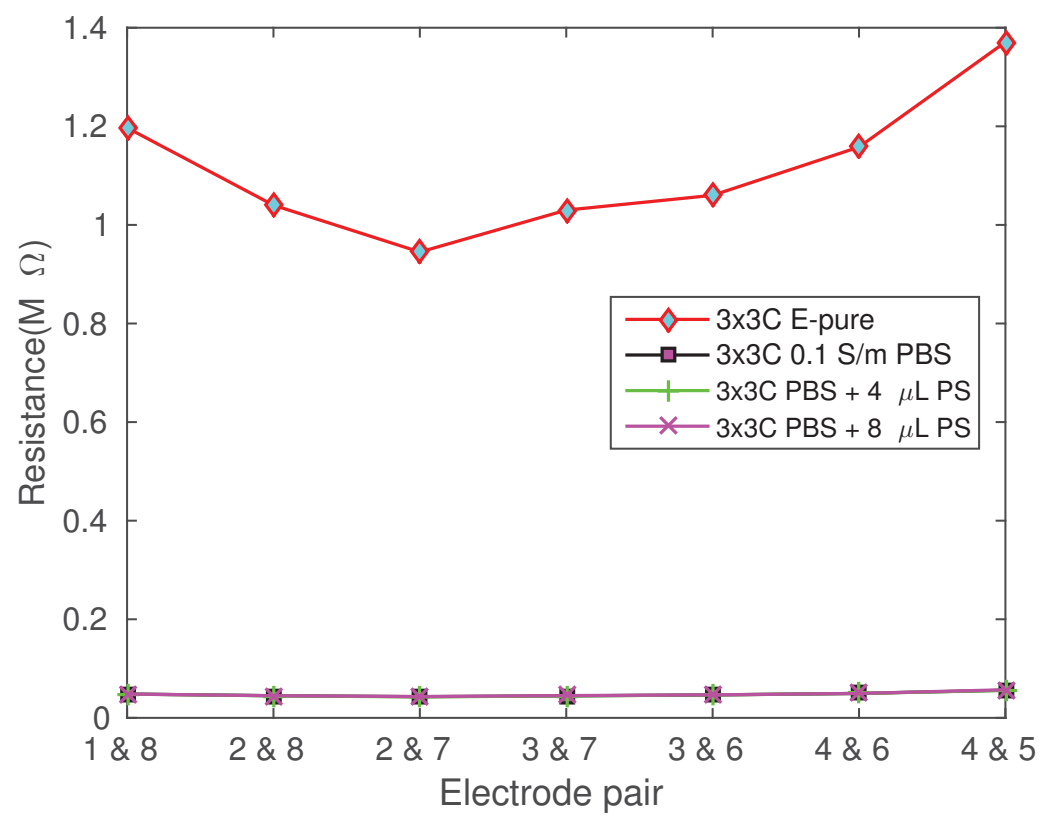

Figure 4.10: Difference in resistance with and without PS beads for 3Circular device showing inconsistent data. This was atrributed to a pure resistance measurement not taking into account all factor at play, leading to a need to gather data on phase angle and impedance. This measurement was done in collaboration with Dr. Hector Moncada.

to the measurement. This data is detailed in Table 4.4.

The phase angle of the dry measurement adhered well to the expected value of $90^{\circ}$. For PBS, it was seen that the phase angle was closer to $45^{\circ}$, which implied a measurement that was almost equally distributed between capacitive and inductive domains. It was concluded that the negative value of capacitance in most cases was a result of poor contact between probe tips and contact pads, and fabrication faults in the device, such as broken leads and inefficient contacts. Using just a capacitance measurement, information about the content of the solution could be gathered, i.e., if it was just PBS or PBS with RBCs. 


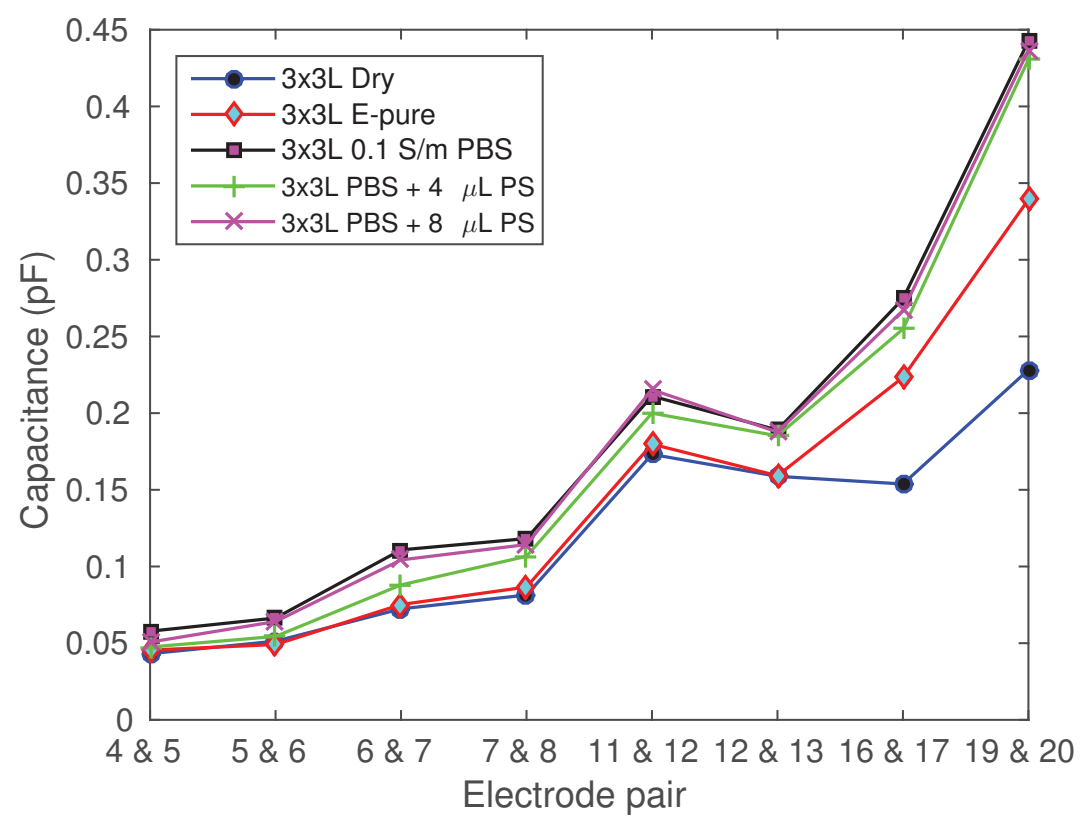

Figure 4.11: Difference in capacitance with and without PS beads for 3Linear device showing discernible difference in values for different analytes. This measurement was done in collaboration with Dr. Hector Moncada.

This data would help optimize the location and design of sensing electrodes to ensure that they could be embedded in a device that does a frequency sweep and collects data on crossover frequencies by sensing the location of RBCs at a particular instant of time. 


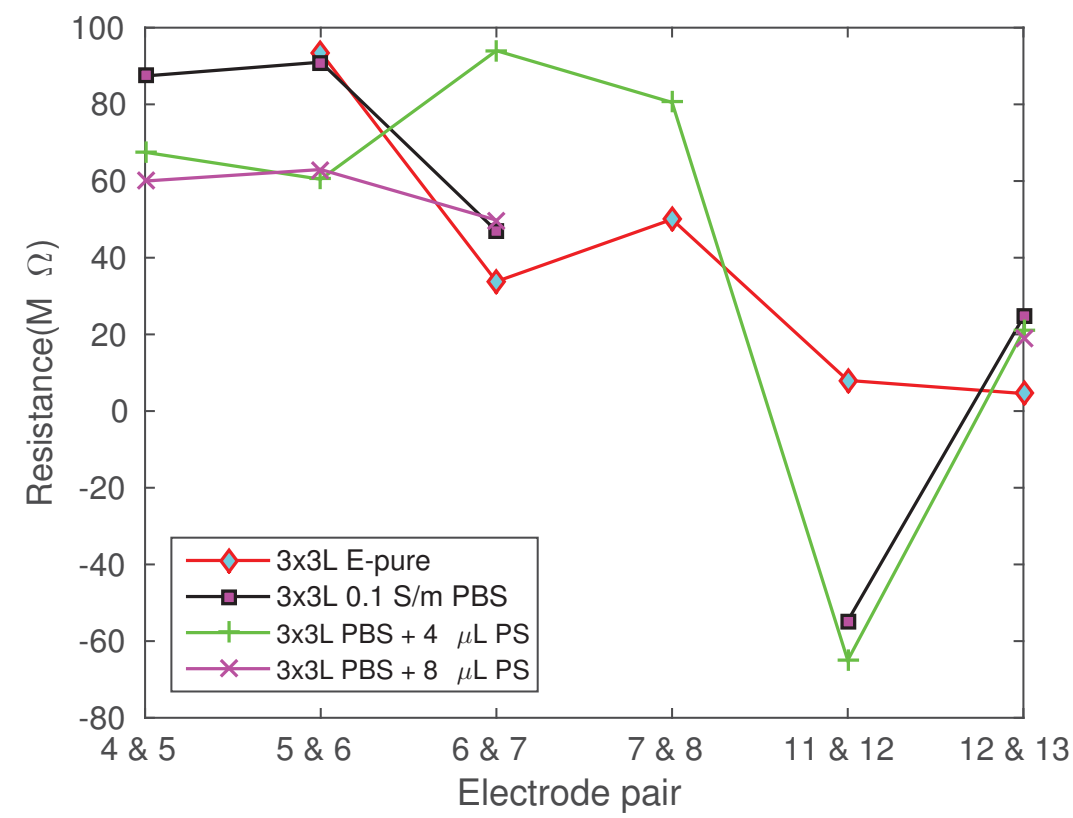

Figure 4.12: Difference in resistance with and without PS beads for 3Linear device showing inconsistent data attributed to bad contact behavior. This measurement was done in collaboration with Dr. Hector Moncada. 
Table 4.1

Mean and Standard Deviation Capacitance and Resistance values of 3Circular

\begin{tabular}{|c|c|c|c|c|c|c|}
\hline Electrode Pair & $\mathrm{Cp}(\mathrm{pF})$ & Cp - mean $(\mathrm{pF})$ & Cp- std dev & $\operatorname{Rp}(\mathrm{M} \Omega)$ & $\mathrm{Rp}-\operatorname{mean}(\mathrm{M} \Omega)$ & Rp - std dev \\
\hline $1 \& 8$ & $\begin{array}{l}0.0388 \\
0.0363 \\
0.0385 \\
0.0363 \\
0.0374\end{array}$ & 0.03746 & 0.00118025 & $\begin{array}{l}- \\
- \\
- \\
- \\
-\end{array}$ & - & - \\
\hline $2 \& 8$ & $\begin{array}{l}0.2083 \\
0.2163 \\
0.2161 \\
0.2188 \\
0.2155\end{array}$ & 0.215 & 0.00395221 & $\begin{array}{l}1.086 \\
1.064 \\
1.038 \\
1.016 \\
0.996\end{array}$ & 1.04 & 0.03608324 \\
\hline $2 \& 7$ & $\begin{array}{c}0.379 \\
0.442 \\
0.4368 \\
0.4562 \\
0.424\end{array}$ & 0.4276 & 0.02951644 & $\begin{array}{c}44.66 \\
42.68 \\
42.8 \\
42.34 \\
42.64\end{array}$ & 43.024 & 0.93009677 \\
\hline $3 \& 7$ & $\begin{array}{c}0.4118 \\
0.4112 \\
0.413 \\
0.4308 \\
0.4255\end{array}$ & 0.41846 & 0.00906521 & $\begin{array}{l}44.67 \\
44.78 \\
44.65 \\
44.26 \\
44.24\end{array}$ & 44.52 & 0.25149553 \\
\hline $3 \& 6$ & $\begin{array}{c}0.406 \\
0.4049 \\
0.4044 \\
0.398 \\
0.4059\end{array}$ & 0.40384 & 0.00333362 & $\begin{array}{c}46.79 \\
46.77 \\
46.69 \\
46.8 \\
46.57\end{array}$ & 46.724 & 0.09633276 \\
\hline $4 \& 6$ & $\begin{array}{l}0.3403 \\
0.3523 \\
0.3549 \\
0.3615 \\
0.3604\end{array}$ & 0.35388 & 0.00849306 & $\begin{array}{l}49.78 \\
49.56 \\
49.62 \\
49.38 \\
49.26\end{array}$ & 49.52 & 0.20396078 \\
\hline $4 \& 5$ & $\begin{array}{l}0.2754 \\
0.2818 \\
0.2759 \\
0.2775 \\
0.2861 \\
\end{array}$ & 0.27934 & 0.00454126 & $\begin{array}{l}56.23 \\
56.07 \\
56.01 \\
55.95 \\
55.79\end{array}$ & 56.01 & 0.16124515 \\
\hline
\end{tabular}


Table 4.2

Mean and Standard Deviation Capacitance and Resistance values of 3Linear

\begin{tabular}{|c|c|c|c|c|c|c|}
\hline Electrode Pair & $\mathrm{Cp}(\mathrm{pF})$ & $\mathrm{Cp}-$ mean $(\mathrm{pF})$ & Cp- std dev & $\operatorname{Rp}(\mathrm{M} \Omega)$ & $\mathrm{Rp}-\operatorname{mean}(\mathrm{M} \Omega)$ & Rp - std dev \\
\hline $4 \& 5$ & $\begin{array}{l}0.0395 \\
0.0442 \\
0.0445 \\
0.0437 \\
0.0439\end{array}$ & 0.04316 & 0.002068333 & $\begin{array}{l}- \\
- \\
- \\
- \\
-\end{array}$ & - & - \\
\hline $5 \& 6$ & $\begin{array}{l}0.0564 \\
0.0519 \\
0.0286 \\
0.0468 \\
0.0619\end{array}$ & 0.04912 & 0.012752529 & $\begin{array}{l}- \\
- \\
- \\
- \\
-\end{array}$ & - & - \\
\hline $6 \& 7$ & $\begin{array}{l}0.0985 \\
0.1131 \\
0.1188 \\
0.1146 \\
0.1092 \\
\end{array}$ & 0.11084 & 0.007706685 & $\begin{array}{l}95 \\
99 \\
95 \\
80 \\
68 \\
\end{array}$ & 87.4 & 13.04990421 \\
\hline $7 \& 8$ & $\begin{array}{l}0.0993 \\
0.1113 \\
0.1013 \\
0.1128 \\
0.1085\end{array}$ & 0.10664 & 0.006031418 & $\begin{array}{c}74.6 \\
55 \\
55 \\
58.3 \\
59.4 \\
\end{array}$ & 60.46 & 8.144814301 \\
\hline $11 \& 12$ & $\begin{array}{l}0.2154 \\
0.2079 \\
0.2177 \\
0.2141 \\
0.2213\end{array}$ & 0.21528 & 0.004948939 & $\begin{array}{l}62 \\
61 \\
50 \\
50 \\
26 \\
\end{array}$ & 49.8 & 14.49827576 \\
\hline $12 \& 13$ & $\begin{array}{l}0.1754 \\
0.1814 \\
0.1889 \\
0.1872 \\
0.1931\end{array}$ & 0.1852 & 0.006902536 & $\begin{array}{c}- \\
- \\
- \\
94 \\
67 \\
\end{array}$ & 80.5 & 19.09188309 \\
\hline $16 \& 17$ & $\begin{array}{l}0.2714 \\
0.2776 \\
0.2734 \\
0.2737 \\
0.2765\end{array}$ & 0.27452 & 0.002503398 & $\begin{array}{c}-57 \\
-45 \\
-56 \\
-61.5 \\
-54\end{array}$ & -54.7 & 6.078651166 \\
\hline $19 \& 20$ & $\begin{array}{l}0.3387 \\
0.3281 \\
0.3393 \\
0.3481 \\
0.3422 \\
\end{array}$ & 0.33928 & 0.007274064 & $\begin{array}{c}4.67 \\
4.65 \\
4.61 \\
4.4 \\
4.53 \\
\end{array}$ & 4.572 & 0.110090872 \\
\hline
\end{tabular}




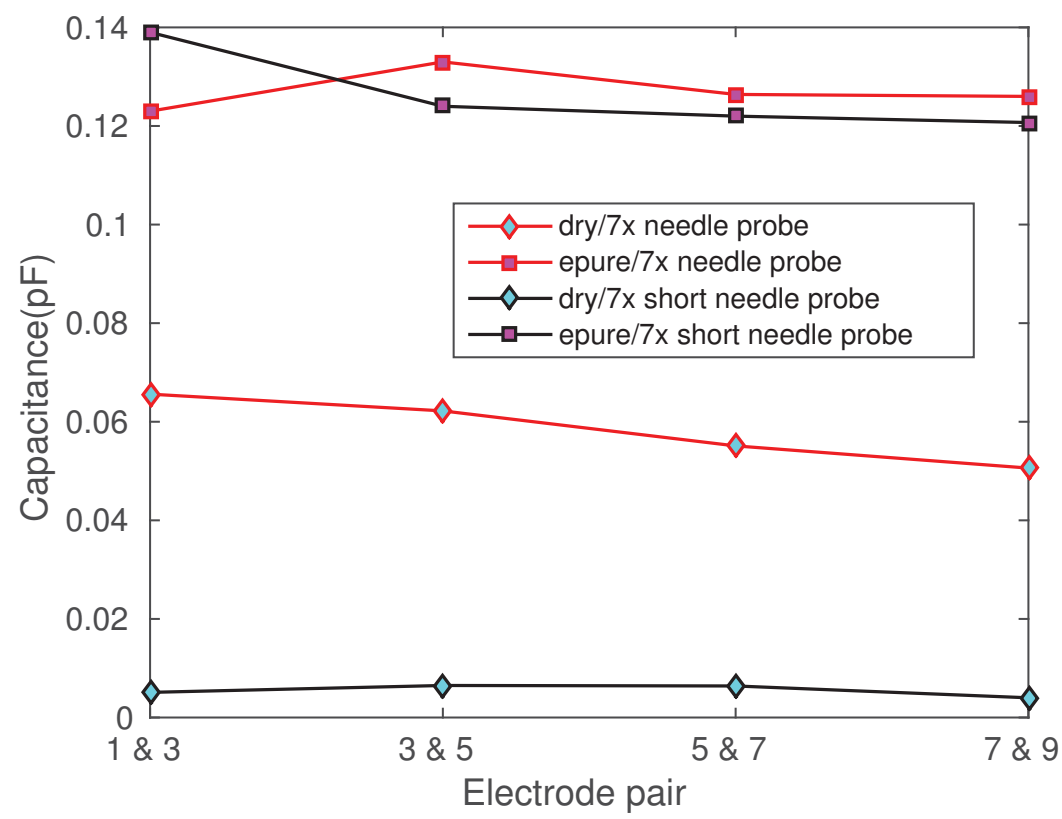

Figure 4.13: Comparison between different probe needles $7 \mathrm{x}$ and $7 \mathrm{x}$ short while performing the same measurement showing $7 \mathrm{x}$ needles yielding higher values of capacitance. This measurement was done in collaboration with Dr. Hector Moncada.

Table 4.3

Impedance data for 3Linear device (standard deviation shown in italics)

\begin{tabular}{|c|c|c|c|c|c|c|c|c|c|c|c|}
\hline \multicolumn{3}{|c|}{ Impedance $(\mathrm{M} \Omega)$} & \multicolumn{3}{|c|}{$0.01 \mathrm{~S} / \mathrm{m}$} & \multicolumn{3}{|c|}{$0.05 \mathrm{~S} / \mathrm{m}$} & \multicolumn{3}{|c|}{$0.1 \mathrm{~S} / \mathrm{m}$} \\
\hline & Dry & E-pure & PBS & $\operatorname{RBC}(1: 130)$ & $\mathrm{RBC}(1: 65)$ & PBS & $\operatorname{RBC}(1: 130)$ & $\operatorname{RBC}(1: 65)$ & PBS & $\operatorname{RBC}(1: 130)$ & $\operatorname{RBC}(1: 65)$ \\
\hline $1 \mathrm{kHz}$ & - & 26.54 & 10.32 & - & - & 5.985 & 3.200 & 3.409 & 4.893 & 7.91 & 6.895 \\
\hline $1 \mathrm{~V}$ & - & 5.2589 & 0.827 & - & - & 1.783 & 0.1952 & 0.1891 & 1.6549 & 1.8663 & 1.4432 \\
\hline $32 \mathrm{kHz}$ & 11.8263 & 2.1538 & 1.2775 & 7.1114 & 7.3786 & 0.452 & 0.2863 & 0.5101 & 0.2819 & 0.5127 & 0.5474 \\
\hline $1 \mathrm{~V}$ & 1.0988 & 0.738 & 0.1506 & 0.7512 & 0.6937 & 0.0725 & 0.0204 & 0.0236 & 0.0768 & 0.1016 & 0.1003 \\
\hline $100 \mathrm{kHz}$ & 11.786 & 2.0390 & 1.2643 & 3.0271 & 3.1057 & 100.69 & 0.4969 & 0.5039 & 0.2977 & 0.4865 & 0.3945 \\
\hline $100 \mathrm{mV}$ & 0.5662 & 0.4216 & 0.0674 & 0.1301 & 0.1189 & 2.1171 & 0.0353 & 0.0262 & 0.0486 & 0.0656 & 0.0592 \\
\hline $500 \mathrm{kHz}$ & 2.3119 & 1.1441 & 0.9051 & 1.1271 & 1.1629 & 0.462 & 0.3801 & 0.4246 & 0.102 & 0.1243 & 0.1553 \\
\hline $100 \mathrm{mV}$ & 0.12 & 1.1602 & 0.034 & 0.0506 & 0.0350 & 0.0429 & 0.0163 & 0.0127 & 0.0091 & 0.0102 & 0.0111 \\
\hline
\end{tabular}

Table 4.4

Phase Angle data for 3Linear device (standard deviation shown in italics)

\begin{tabular}{|c|c|c|c|c|c|c|c|c|c|c|c|}
\hline \multicolumn{3}{|c|}{ Phase angle $\left(^{\circ}\right)$} & \multicolumn{3}{|c|}{$0.01 \mathrm{~S} / \mathrm{m}$} & \multicolumn{3}{|c|}{$0.05 \mathrm{~S} / \mathrm{m}$} & \multicolumn{3}{|c|}{$0.1 \mathrm{~S} / \mathrm{m}$} \\
\hline & Dry & E-pure & PBS & $\mathrm{RBC}(1: 130)$ & $\mathrm{RBC}(1: 65)$ & PBS & $\mathrm{RBC}(1: 130)$ & $\mathrm{RBC}(1: 65)$ & PBS & $\mathrm{RBC}(1: 130)$ & $\mathrm{RBC}(1: 65)$ \\
\hline $1 \mathrm{kHz}$ & -89.70 & -62.26 & -64.95 & -72.2714 & -70.6286 & -69.58 & -74.03 & -71.18 & -72.21 & -70.8 & -71.45 \\
\hline $1 \mathrm{~V}$ & 0.1886 & 7.9246 & 0.1269 & 1.7347 & 1.7988 & 0.4077 & 0.4218 & 0.49 & 0.5971 & 0.8667 & 1.0277 \\
\hline $32 \mathrm{kHz}$ & -89.77 & -32.13 & -33.86 & -79.6 & -76.1429 & -49.66 & -42.14 & -34.36 & -58.39 & -63.33 & -57.76 \\
\hline $1 \mathrm{~V}$ & 0.0483 & 2.0476 & 3.4224 & 3.3591 & 3.7797 & 3.7256 & 1.2607 & 1.4998 & 2.9314 & 2.05 & 2.28 \\
\hline $100 \mathrm{kHz}$ & -89.77 & -31.62 & -27.63 & -62.9143 & -63.0429 & -45.89 & -30.37 & -26.08 & -62.78 & $\begin{array}{l}-66.31 \\
\end{array}$ & -58.85 \\
\hline $100 \mathrm{mV}$ & 0.1059 & 2.0411 & 1.7901 & 2.1027 & 3.3615 & 4.2506 & 2.8237 & 2.9306 & 3.1909 & 2.9516 & 3.6339 \\
\hline $500 \mathrm{kHz}$ & -89.92 & -44.547 & -39.61 & -64.5429 & -69.5 & -45.89 & -22.29 & -22.74 & -41.85 & -45.56 & -37.41 \\
\hline $100 \mathrm{mV}$ & 0.0355 & 15.4075 & 1.3544 & 0.4237 & 1.4283 & 4.2506 & 1.3329 & 1.3672 & 5.5557 & 4.8195 & 4.5742 \\
\hline
\end{tabular}




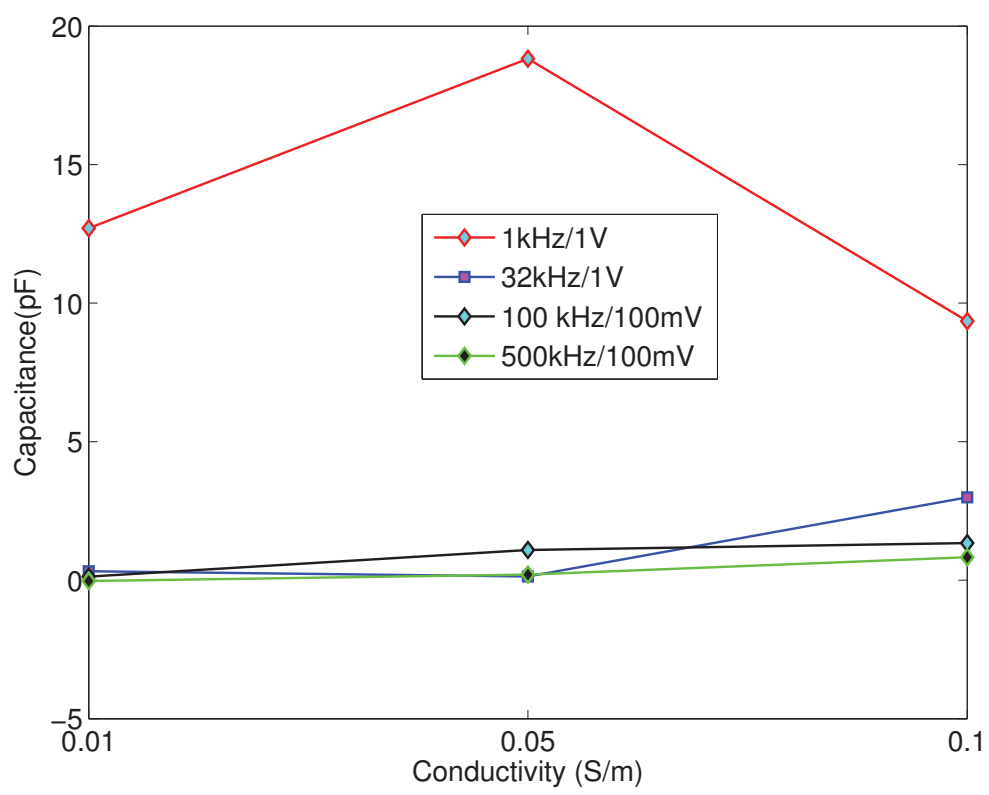

Figure 4.14: Differential measurement of PBS and RBCs (1:65 concentration) showing higher sensitivity at $1 \mathrm{kHz}, 1 \mathrm{~V}$ for a solution conductivity of $0.05 \mathrm{~S} / \mathrm{m}$. This measurement was done in collaboration with Dr. Hector Moncada. 


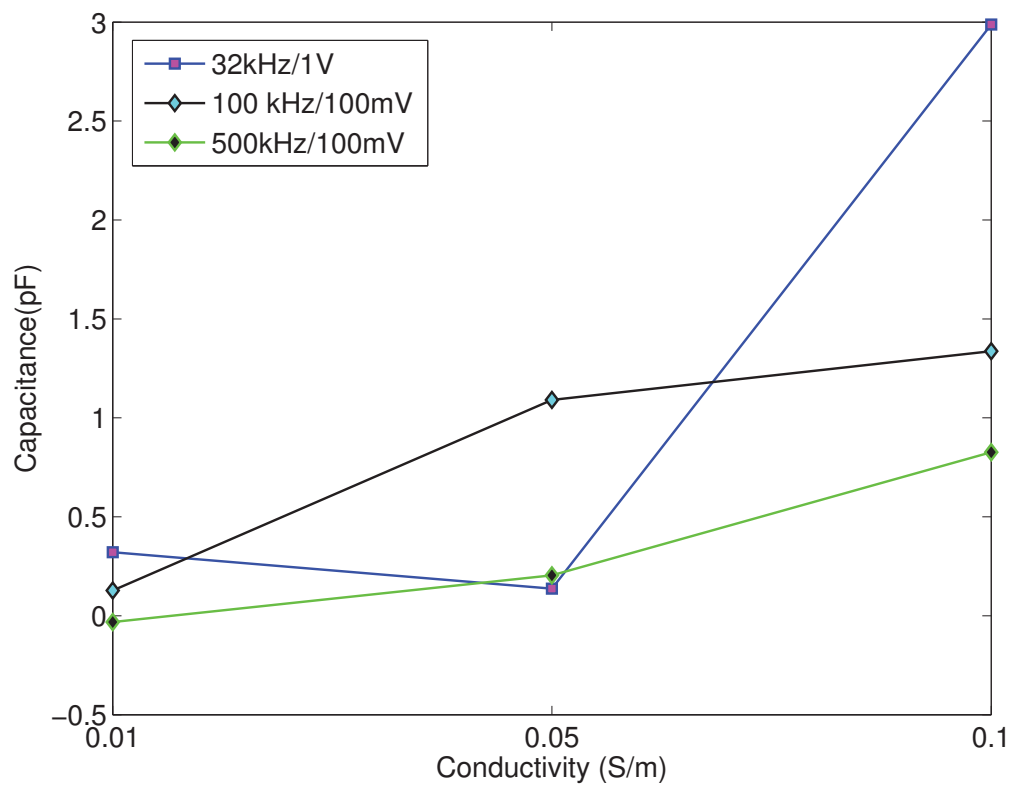

Figure 4.15: Differential measurement of PBS and RBCs (1:65 concentration) showing highest sensitivity at $32 \mathrm{kHz}, 1 \mathrm{~V}$ for a solution conductivity of $0.01 \mathrm{~S} / \mathrm{m}$ - the ideal case for alpha prototype. This measurement was done in collaboration with Dr. Hector Moncada. 


\section{Chapter 5}

\section{Conclusions and Future Work}

\subsection{Conclusions}

The goal of the research conducted for this thesis was to create a blood typing sensor that would obviate the need for optical measurements. There was a need to create a portable, handheld device that was capable of typing blood in order to expedite the process by making use of an electrical measurement instead of an optical measurement. With this new approach, a pathology lab, reagents and a trained laboratory technician are all not needed. Initial results suggest that process flow described for these sensors may lead to a more streamlined automation of measurement and the 
device could be used in by a first response team in a triage situation. Also, an electrical measurement is less subjective and depends purely on where the cells are located at a particular frequency. The scope for human error is greatly reduced.

Chapter two described the design and the model of a sensing array that can be used as the sensing region for these blood cells. The model, made in COMSOL 4.4 showed the expected response under various conditions. It showed the effect of different frequencies on the sensing electrodes as well as the drive. The effect of passivation had also been studied. It was noticed from the COMSOL simulations that the sensing array generates a strong enough field to affect the DEP manipulation of the RBCs in such a way that it is not solely dependent on the drive field. This led to an understanding that switching between drive electrodes and sensing electrodes would be necessary to increase measurement accuracy.

Chapter three described fabrication techniques for this biosensor. An effort was made to make the entire procedure industry compliant by using standardized processes and tools. Four different masking layers have been described and implemented. It was concluded that devices could be successfully fabricated using these methods and a high degree of uniformity was required during deposition and etch processes. It was seen that an evaporative deposition process combined with a wet metal etch yielded best results. Different means of passivation were considered and hafnia was the most robust and facilitated the best bonding with PDMS. 
Chapter four described testing procedures, results and analysis. The live tissue in the red blood cells had to be first deactivated and then its response in an electric field was recorded using capacitance, resistance, impedance and phase angle measurements. It was seen that there was a difference between these measured values with different reagents. Dry measurements yielded the lowest capacitance value. Measurements made using epure water showed a resistance of $18 \mathrm{M} \Omega$ which was consistent with the measured resistance. There was a difference seen between the capacitances of electrodes when a cell was present and when it was not, i.e., the testing with PBS only yielded different values of capacitance when compared to PBS with RBCs. The effect of different solution conductivites with varying concentration of RBCs was studied as well and it was seen that at $32 \mathrm{kHz}$ at $1 \mathrm{~V}$, the highest sensitivity to a differential measurement was possible for a solution conductivity of $0.1 \mathrm{~S} / \mathrm{m}$. This difference was $3 \mathrm{pF}$, well within the range of available instrumentation. Testing of impedance and phase angle yielded data that further explained the behavior of the device as a capacitive type sensor and was helpful in debugging previously recorded results.

It was concluded that a process to sense red blood cells in the presence of an electric field was defined and successfully tested, using an array of sensing electrodes.

Objective one of this thesis was to design and interface parallel ITO sensing electrodes with microfluidic chamber to sense cell location. The successful fabrication 
and modeling of this device satisfies this objective.

Objective two was to test ITO sensing electrodes concurrent with DEP manipulation of RBCs to ascertain response times and interference. Wafer scale fabrication of the devices and the subsequent dicing to test the dies with a card edge connector on a board containing external circuitry satisfies this objective. RBCs were pickled and used for initial testing using the probe station as described in Chapter 4, but the DEP testing using the drive electrodes on the external prototype is still in progress.

\section{$5.2 \quad$ Future Work}

There are certain aspects of the design and fabrication of the thesis device that need further investigation. The process flow for fabrication described in the thesis primarily uses liftoff instead of an etch process. Although initial testing using an etch process is underway, a process flow needs to be defined for patterning of the metallization layer using Chrome and Gold etchant. Preliminary testing of this etch process involves patterning the metallization layer first, using an evaporated blanket coating of $25 \mathrm{~nm}$ of $\mathrm{Cr}$ and $100 \mathrm{~nm} \mathrm{Au}$. The metallization layer was etched and then aligned to the sensing electrodes. The subsequent steps would need a liftoff. ITO etches in aqua regia, which would damage the underlying layers and to an extent, the substrate. To make this device industry complaint for production, an etch process is essential. 
The location of the electrodes can be revised based on DEP test data. Simulation results as shown in Chapter 2 demonstrate that the sensing electrodes have an impact on the movement of RBCs in the presence of a field from the drive electrodes. Keeping in mind the altered motion of cells, sensing arrays could be located in optimized locations.

There is a need to alter the design of the sensing electrodes to create an array of pixels to improve sensitivity. These pixels can be strategically placed in parts of the chamber that show strongest DEP response. This would require measurement of lower values of capacitance and would in turn necessitate use of more sensitive measurement methods and changes on on-board circuitry. A highly sensitive capacitance-to-digital converter would be required to extract a small value of capacitance from the noise, and on board circuitry would need to be changed to account for the same.

A major part of this research demonstrates a methodology that can be used to detect any particle that exhibits a DEP response. This technology could be used for detection of cells, pathogens and polar molecules. There is a need for rapid objective sensing that adheres well to a fully automated process. This thesis presents preliminary data on electrical sensing of red blood cells. However, the same technology could be used for a variety of purposes.

The modeling and experimental knowledge gained through the research has the potential to be applied to various other biosensors. This very project has been shown to 
be conceivable at a moderately large scale and could be implemented commercially to create a rapid sensing blood typing device that does not use reagents or require laboratory support. 


\section{References}

[1] http://www.zeonex.com/optics.aspx. Optical transmission of Zeonex in visible and near-visible range; Zeonex: The Internet, 2015.

[2] Nolte, E.; McKee, M. Does health care save lives? Avoidable mortality revisited.; The Nuffield Trust, 2004.

[3] Gascoyne, P. R.; Wang, X.-B.; Huang, Y.; Becker, F. F. Industry Applications, IEEE Transactions on $1997,33(3), 670-678$.

[4] Gascoyne, P.; Pethig, R.; Satayavivad, J.; Becker, F. F.; Ruchirawat, M. Biochimica et Biophysica Acta (BBA)-Biomembranes 1997, 1323(2), 240-252.

[5] Daniels, G.; Fletcher, A.; Garratty, G.; Henry, S.; Jørgensen, J.; Judd, W.; Levene, C.; Lomas-Francis, C.; Moulds, J.; Moulds, J.; others. Vox sanguinis 2004, 87(4), 304-316.

[6] Landsteiner, K. The specificity of serological reactions; Courier Corporation, 1990. 
[7] Landsteiner, K.; Wiener, A. S. Experimental Biology and Medicine 1940, 43(1), $223-223$.

[8] http://www.api pt.com/Reference/Commentary/2013Cbbank.pdf. Immediate Spin Crossmatch; American Proficiency Institute: The Internet, 2013.

[9] Berry-Dortch, S.; Woodside, C.; Boral, L. Transfusion 1985, 25(2), 176-178.

[10] Force, B. B. T. T. Transfusion medicine 2004, 14, 59-73.

[11] Judd, W. J. Vox sanguinis 1998, 74(S2), 409-417.

[12] Pohl, H. A. Journal of Applied Physics 1951, 22(7), 869-871.

[13] Pohl, H. A.; Crane, J. S. Biophysical journal 1971, 11(9), 711-727.

[14] Bhattacharjee, B.; Najjaran, H. Lab on a Chip 2012, 12(21), 4416-4423.

[15] Minerick, A. R.; Zhou, R.; Takhistov, P.; Chang, H.-C. Electrophoresis 2003, $24(21), 3703-3717$.

[16] Kong, J. A. New York, Wiley-Interscience, 1975. 348 p. 1975, 1.

[17] Yang, H. Ph. D. Dissertation 2000.

[18] Leus, V.; Elata, D. Technion-Israel Institute of Technology Technical Report No. ETR-2004-2 2004.

[19] Shah, K.; Singh, J.; Zayegh, A. SPIE Proceedings Vol. 6035: Microelectronics: Design, Technology, and Packaging II 2006. 
[20] Harriott, L. R. Proceedings of the IEEE 2001, 89(3), 366-374.

[21] Pethig, R. Biomicrofluidics 2010, 4(2), 022811.

[22] Leonard, K.; Rutan, E.; Reeves, S.; Pate, A.; Walton, M.; Thompson, S.; Minerick, A. In The 2008 Annual Meeting, 2008.

[23] Granqvist, C.; Hultåker, A. Thin solid films 2002, 411(1), 1-5.

[24] Guillén, C.; Herrero, J. Solar Energy Materials and Solar Cells 2008, 92(8), 938-941.

[25] Meng, L.-j.; Macarico, A.; Martins, R. In MRS Proceedings, Vol. 388, page 379. Cambridge Univ Press, 1995.

[26] Hassler, C.; von Metzen, R. P.; Ruther, P.; Stieglitz, T. Journal of Biomedical Materials Research Part B: Applied Biomaterials 2010, 93(1), 266-274.

[27] Lee, J. H.; Hwang, K. S.; Kim, T. S.; Seong, J. W.; Yoon, K. H.; Ahn, S. Y. Korean Physical Society 2004, 44(5).

[28] Lee, J. H.; Hwang, K. S.; Yoon, K. H.; Kim, T. S.; Ahn, S. Plasma Science, IEEE Transactions on 2004, 32(2), 505-509.

[29] https://www.utdallas.edu/ rar011300/Parylene/ParyleneDepManual.pdf. Parylene Adhesion Promotion Process; University of Texas at Dallas: The Internet, 2015.

[30] Gordon, J. E.; Gagnon, Z.; Chang, H.-C. Biomicrofluidics 2007, 1(4), 044102. 